Florida International University FIU Digital Commons

5-21-2014

\title{
Essays on Migration: Nexus with Policy, Trade, and Development
}

Michael Good

mgood010@fiu.edu

DOI: $10.25148 /$ etd.FI14071116

Follow this and additional works at: https://digitalcommons.fiu.edu/etd

\section{Recommended Citation}

Good, Michael, "Essays on Migration: Nexus with Policy, Trade, and Development" (2014). FIU Electronic Theses and Dissertations. 1450.

https://digitalcommons.fiu.edu/etd/1450 


\title{
FLORIDA INTERNATIONAL UNIVERSITY
}

Miami, Florida

\section{ESSAYS ON MIGRATION: NEXUS WITH POLICY, TRADE, AND DEVELOPMENT}

\author{
A dissertation submitted in partial fulfillment of \\ the requirements for the degree of \\ DOCTOR OF PHILOSOPHY \\ in \\ ECONOMICS \\ by \\ Michael Good
}

2014 
To: Interim Dean Michael R. Heithaus

College of Arts and Science

This dissertation, written by Michael Good, and entitled Essays on Migration: Nexus with Policy, Trade, and Development, having been approved in respect to style and intellectual content, is referred to you for judgment.

We have read this dissertation and recommend that it be approved.

Kazuyuki Miyagiwa

Hakan Yilmazkuday

Hassan Zahedi

Cem Karayalcin, Major Professor

Date of Defense: May 21, 2014

The dissertation of Michael Good is approved.

Interim Dean Michael R. Heithaus

College of Arts and Sciences

Dean Lakshmi N. Reddi

University Graduate School

Florida International University, 2014 
(C) Copyright 2014 by Michael Good

All rights reserved. 


\section{DEDICATION}

I dedicate this dissertation to my wife, Yazmín. Thanks to her constant love, support and encouragement, not only did I discover economics, the completion of this work became possible. 


\section{ACKNOWLEDGMENTS}

I would like to express appreciation to my committee members, Kaz Miyagiwa, Hakan Yilmazkuday, and Hassan Zahedi, for their contribution of time and effort in providing feedback throughout the writing of this dissertation. I give special thanks to my major professor, Cem Karayalcin, for his guidance during these five years of my progression through the Ph.D. program - from class to research to the job search, his willingness to listen and advise has been much appreciated.

Additionally, thank you to the Horowitz Foundation for Social Policy Research, the Broad Fellowship, as well as Florida International University’s Presidential Fellowship, Dissertation Evidence Acquisition Fellowship, and Dissertation Year Fellowship for financial support. 


\title{
ABSTRACT OF THE DISSERTATION \\ ESSAYS ON MIGRATION: NEXUS WITH POLICY, TRADE, AND \\ DEVELOPMENT
}

\author{
by
}

Michael Good

Florida International University, 2014

Miami, Florida

\section{Professor Cem Karayalcin, Major Professor}

This dissertation analyzes the intersection of migration with public policy, international trade, and economic development. The first essay investigates the impact on internal migration for 52 different demographic groups of the recent influx of state omnibus immigration laws targeting undocumented immigrants in the United States. Through a difference-in-differences estimation, I find empirical evidence that while the demographic groups pinpointed as having higher percentages of undocumented immigrants certainly experience population and employment 'outflows' from states implementing these immigration laws, there is a lack of associated 'inflows' for those demographic groups identified by economic theory as being probable substitutes for undocumented immigrants. Several segments designated as probable substitutes actually experience an adverse effect on population and employment.

The second essay examines the effect that migrants have on international trade between states of current residence and states of origin. My analysis provides the first results as to the migration-trade nexus at the state level for both places of destination and origin, relying on a unique data set allowing the mapping of Mexican-born migrants' U.S. 
states of residence to Mexican states of origin. In addition to an augmented gravity model, I employ generalized propensity scores in examining the potential of nonlinearities in the migration-trade relationship, estimating statistically significant elasticities of exports to both in-state and neighboring-state migration.

The third essay analyzes the potentially enormous wage gains that may motivate international migration, an activity which is limited to some extent by governments across the entire world. Freer human mobility and the effects of migration on the migrants themselves have not garnered nearly as much attention as numerous other topics related to the economics of migration. I present novel data collected through household interviews in communities both in Mexico and the United States, comparing the absolute and relative wage gains for interviewees with data from existing Mexican surveys. Migrants indeed stand to collect large net gains; average incomes increase more than fivefold immediately, moving from the lower deciles of origin wage distributions to the top deciles. These results surpass those of some of the most successful current programs of economic development.

\section{TABLE OF CONTENTS}

CHAPTER

PAGE 
I. DO IMMIGRANT OUTFLOWS LEAD TO NATIVE INFLOWS? AN EMPIRICAL ANALYSIS OF THE MIGRATORY RESPONSES TO U.S. STATE IMMIGRATION LEGISLATION

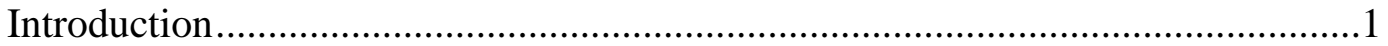

State Immigration Law …….....................................................................

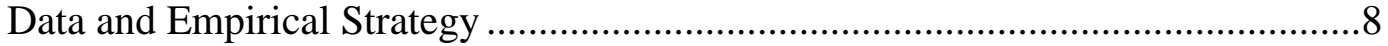

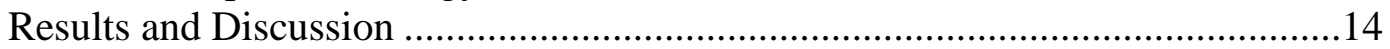

Checks on Initial Estimates............................................................................19

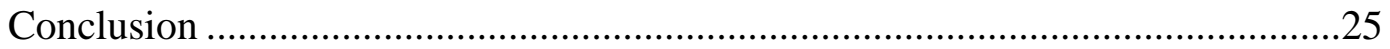

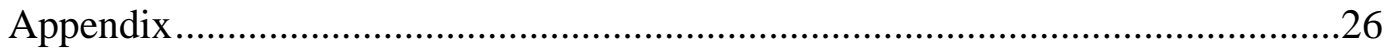

II. GEOGRAPHIC PROXIMITY AND THE PRO-TRADE EFFECT OF MIGRATION: STATE-LEVEL EVIDENCE FROM MEXICAN MIGRANTS IN

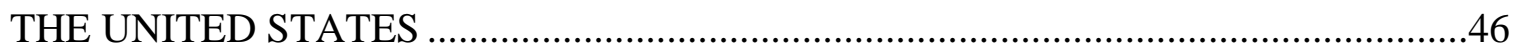

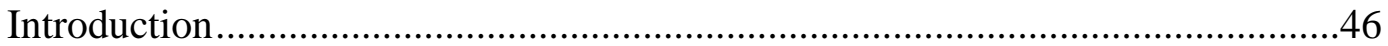

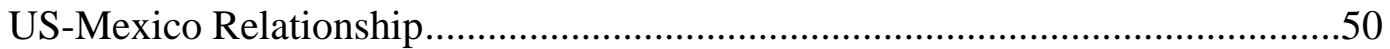

Empirical Strategy and Data ........................................................................53

Results and Discussion .......................................................................................59

Robustness and Sensitivity Checks.....................................................................64

Extension: An Application of Generalized Propensity Scores ................................67

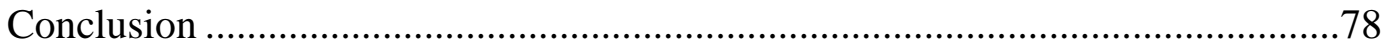

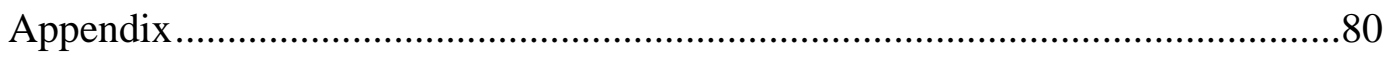

III. WAGE GAPS, MIGRATION, AND DEVELOPMENT: A VIEW FROM BOTH

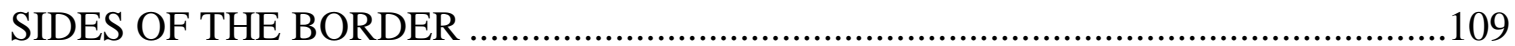

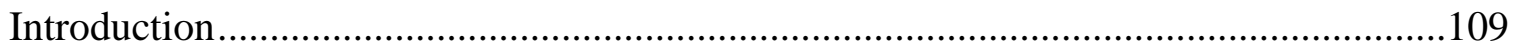

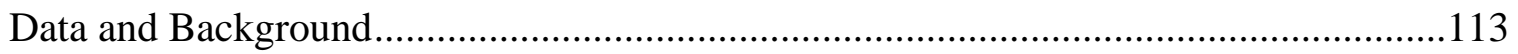

Results and Discussion ...................................................................................117

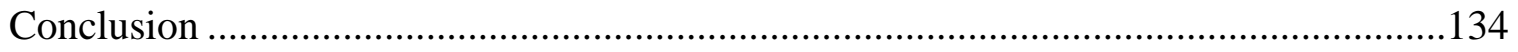

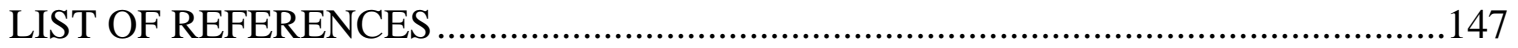

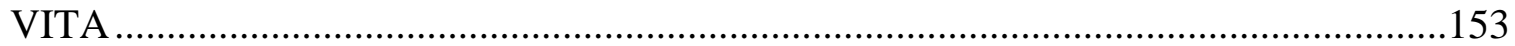




\section{LIST OF TABLES}

TABLES

PAGE

1.1 Enacted state immigration laws: 2005 to 2011 .......................................................30

1.2 Pre-implementation characteristics of states, regional control ....................................31

1.3 Pre-implementation characteristics of states, national control .................................32

1.4 Difference-in-differences estimates of the impact of state omnibus immigration

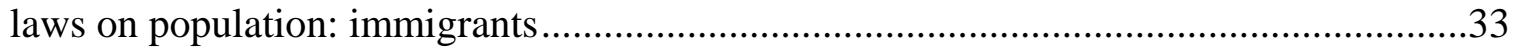

1.5 Difference-in-differences estimates of the impact of state omnibus immigration

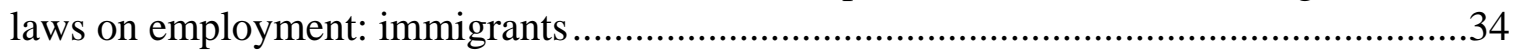

1.6 Difference-in-differences estimates of the impact of state omnibus immigration laws on population: natives............................................................................................35

1.7 Difference-in-differences estimates of the impact of state omnibus immigration

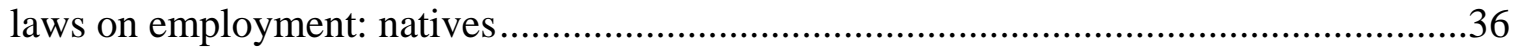

1.8 Difference-in-differences estimates of the impact of state omnibus immigration laws on population and employment (no Oklahoma): immigrants......................................37

1.9 Difference-in-differences estimates of the impact of state omnibus immigration laws on population and employment (no Oklahoma): natives.

1.10 Difference-in-differences estimates of the impact of state omnibus immigration laws on population and employment: immigrants

1.11 Difference-in-differences estimates of the impact of state omnibus immigration laws on population and employment: natives

1.12 Rankings one year before implementation

1.13 Estimates of the impact of the narrowest demographic on state immigration legislation implementation.

2.1 Descriptive statistics - base and alternative samples.

2.2 Coefficient estimates using gravity equation (OLS, state fixed effects)

2.3 Comparison of estimates for the elasticity of exports to migration. 
2.4 Coefficient estimates using gravity equation, alternate samples (OLS, state fixed effects)

$2.5 \gamma_{1}$ and $\gamma_{2}$ with alternative (in)dependent variables (OLS, state fixed effects) ............86

2.6 Coefficient estimates using forwarded exports (OLS, state fixed effects) .................87

2.7 Coefficient estimates using gravity equation, migrant share (OLS, state fixed effects)

2.8 OLS estimation, dependent variable: $\ln$ (migrants) ………..........................................89

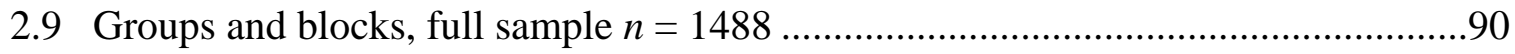

2.10 Groups and blocks, modified common support sample $n=1429 \ldots \ldots \ldots \ldots \ldots \ldots \ldots \ldots \ldots \ldots . . . . .90$

2.11 Three-stage balancing comparison of covariates ........................................................91

2.12 Covariate means: included vs. excluded observations...............................................92

2.13 OLS estimation, dependent variable: $\ln ($ exports) ………..........................................92

A.1 Matrículas consulares registered 2006 to 2010, US states of residence and Mexican states of origin: total of 4,659,656

A.2 Migration and trade in top US states of Mexican migrant residence.

A.3 OLS estimation, dependent variable: $\ln$ (migrants) …….............................................95

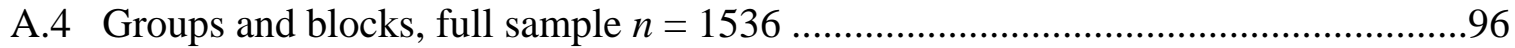

A.5 Groups and blocks, modified common support sample $n=1478 \ldots \ldots \ldots \ldots \ldots \ldots \ldots \ldots \ldots \ldots \ldots . . .96$

A.6 Three-stage balancing comparison of covariates .......................................................97

A.7 OLS estimation, dependent variable: $\ln$ (exports) ………........................................98

A.8 OLS estimation, dependent variable: $\ln$ (migrants) ……...........................................98

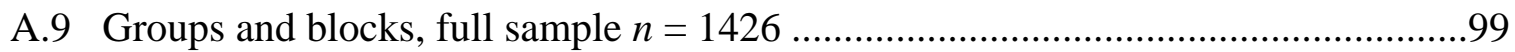

A.10 Groups and blocks, modified common support sample $n=1380 \ldots \ldots \ldots \ldots \ldots \ldots \ldots \ldots . . . . . . . . .99$

A.11 Three-stage balancing comparison of covariates ..................................................100 
A.12 OLS estimation, dependent variable: $\ln$ (exports)

.101

A.13 State-state observations excluded by modified common support condition, starting sample of $n=1488$.

A.14 State-state observations excluded by modified common support condition, starting sample of $n=1536$.

A.15 State-state observations excluded by modified common support condition, starting sample of $n=1426$.

3.1 Summary statistics of migrant samples.

3.2 Migrant wage gains

3.3 Break-even hours

3.4 Wage gains and subjective well-being

3.5 Length of time since migration and subjective well-being.

3.6 Simple correlations: observable characteristics and gains from migration .140

3.7 OLS regression results 140 


\section{LIST OF FIGURES}

FIGURES

PAGE

1.1 Population before and after implementation: narrowest demographic .42

1.2 Employment before and after implementation: narrowest demographic. .43

1.3 Population at implementation: narrowest demographic .44

1.4 Employment at implementation: narrowest demographic .45

2.1 Percentage distribution of matrículas consulares vs. U.S. Census 105

2.2 Mexican states average education and migration .105

2.3 Dose response function and treatment effect function, $n=1429$ 106

2.4 Marginal contributions for migrants $>500$ .106

2.5 Marginal contributions for migrants $<500$ .107

A.1 Migration and trade, simple correlation

A.2 Dose response function and treatment effect function, $n=1478$ .108

A.3 Dose response function and treatment effect function, $n=1380$ 108

3.1 HIMEX wages before and after migration. .141

3.2 HIMEX wage gains from migration .141

3.3 HIMEX wage ratios

3.4 HIUS wages before and after migration 142

3.5 HIUS wage gains from migration

3.6 HIUS wage ratios .143

3.7 EMIF wages 144

3.8 EMIF wage ratios... 144

3.9 HIMEX wages .145 


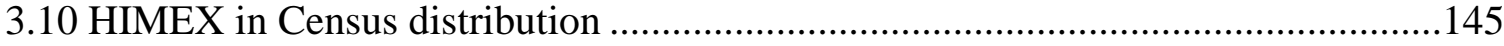

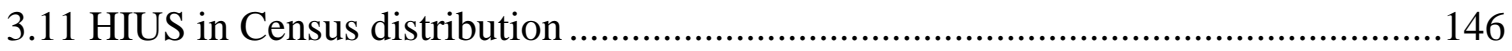




\section{ABBREVIATIONS AND ACRONYMS}

\begin{tabular}{|c|c|}
\hline APR & Artal-Tur et al. \\
\hline BEA & Bureau of Economic Analysis \\
\hline CMP & Clemens et al. \\
\hline CONEVAL & Consejo Nacional de Evaluación de la Política de Desarrollo Social \\
\hline CPI-U & Consumer price index - urban \\
\hline CPS & Current Population Survey \\
\hline EMIF & Encuesta sobre migración en la frontera norte de México \\
\hline EVN & Egger et al. \\
\hline GPS & Generalized propensity squares \\
\hline GSP & Gross state product \\
\hline HIMEX & Household interviews - Mexico sample \\
\hline HIUS & Household interviews - United States sample \\
\hline HS & Herander and Saavedra \\
\hline IME & Instituto de los Mexicanos en el Exterior \\
\hline INEGI & Instituto Nacional de Estadística y Geografía \\
\hline MMP & Mexican Migration Project \\
\hline NSL & National Survey of Latinos \\
\hline OECD & Organization for Economic Cooperation and Development \\
\hline OLS & Ordinary least squares \\
\hline P\$ & Purchasing Power Parity dollars \\
\hline PUMA & Public Use Microdata Area \\
\hline SR & Serrano and Requeña \\
\hline
\end{tabular}




\section{CHAPTER I}

DO IMMIGRANT OUTFLOWS LEAD TO NATIVE INFLOWS?

\section{AN EMPIRICAL ANALYSIS OF THE MIGRATORY RESPONSES TO U.S. STATE IMMIGRATION LEGISLATION}

\section{Introduction}

The recent influx of state immigration legislation in the United States provides an ideal quasi-experimental setting appropriate for examining the connection between immigration and internal migration. Since 2006, eleven different states have enacted fourteen omnibus immigration laws, implementing broad restrictions in relation to issues affecting immigrants such as work authorization, public program benefits, education, human trafficking and the transport and harbor of unauthorized immigrants, identification and driver's license policies, and document-carrying policies. A simple analysis of the incentives created by these wide-reaching omnibus laws leads to an expectation of outflows of the undocumented immigrant population from those states implementing immigration laws, as well as possible inflows among other demographics not specifically targeted by the new laws. Anecdotal evidence certainly provides backing to this expectation of immigrant outflows, as there are numerous reports of large out-migrations, especially of the undocumented population, in states which have recently put omnibus immigration laws into practice. ${ }^{1}$ However, much of the same anecdotal evidence also points to a lack of inflows of other demographic groups into the immigrant-vacated jobs, leading to a shortage of workers in certain areas where the outflows have been particularly strong. The anecdotal

\footnotetext{
${ }^{1}$ See Robertson (2011) for one of many articles documenting this migratory response.
} 
evidence begs the empirical analysis of a simple yet important question, which is the focus of the present study: Do immigrant outflows lead to native inflows?

The approach to attack this question utilizes a straightforward, two-step process. First, I use microdata from the Current Population Survey (CPS) to analyze in great detail the pre- and post-implementation numbers relating to both population and employment of demographic groups targeted by the state immigration laws. By use of a difference-indifferences estimation that exploits the natural experiment setting and employs a treatment and control group (the treatment being the implementation of the state immigration law), I am able to verify if there truly are immigrant outflows in response to the change in state policy. Second, using the same microdata along with the treatment and control process, I analyze the pre- and post-implementation numbers of the demographic groups not targeted by the state immigration laws, permitting the identification of any native (or documented immigrant) population or worker inflows (outflows) in those states experiencing targeted immigrant outflows.

The importance of the study's findings is twofold. First, the documentation of the actual migratory response to the surge of state immigration laws is important in its own right. While much anecdotal evidence has been accumulated, detailed empirical analyses are lacking, possibly partly due to the relative newness of this trend in immigration legislation and the difficulty inherent in identifying the undocumented population. Second, there is an established literature examining the relationship between immigration and internal migration responses. However, despite numerous studies, this debate laden with key policy implications still has no clear victor. The present study contributes to the ongoing debate by providing additional rigorous empirical evidence as to the relationship 
between immigration and internal migration responses. My analysis indicates that while the demographic groups pinpointed as having higher percentages of undocumented individuals indeed experience population and employment outflows from states implementing these immigration laws, there is a lack of associated inflows for those demographic groups identified by economic theory as being probable substitutes for undocumented immigrants. Although minimal substitution is present, several segments of the population designated as probable substitutes actually experience an adverse effect on population and employment.

To my knowledge, this is the first research exploiting the implementation of state omnibus immigration laws to explicitly examine the question posited above: Do immigrant outflows lead to native inflows? Card and Dinardo (2000) asks the question 'Do immigrant inflows lead to native outflows?' in the very title of the article - the experiment provided by the implementation of the current state immigration legislation now allows for a natural examination of the opposite side of their question, arguably just as important. The chapter is most related to Raphael and Ronconi (2009), which finds a significant out-migration of Hispanic immigrants from states passing immigration legislation. However, while similar in approach, their article examines only immigration laws specifically targeting employment and the sample is limited to pre-2009 implementation of laws, excluding a majority of this paper's richer sample.

After providing a brief background of recent U.S. state immigration law and a review of the relevant economic theory, I give specifics as to the data and my empirical strategy. I discuss the results of the difference-in-differences estimations for the various 
demographic groups, finally checking for sensitivity and robustness before a brief conclusion.

\section{State Immigration Law}

\section{A brief overview}

Evident from the statistics provided by the National Conference of State Legislatures, the recent growth in the number of state immigration laws enacted in the United States is astounding. Table 1.1 outlines the number of immigration laws by category passed by state legislatures during the period of 2005 to 2011, the number vetoed by governors, and the number ultimately adopted by states. ${ }^{2} \mathrm{~A}$ majority of the laws target a specific issue in relation to undocumented immigrants, such as work authorization, public program benefits, or identification and licenses. While these focused laws certainly lower the incentive for an undocumented immigrant to reside in the respective state (some much more than others), a handful of states have implemented omnibus laws that address multiple issues at the same time. These broad-reaching laws theoretically have the largest effect on any outflows of immigrants, due to their nature of affecting not only employment opportunities, but also possibly limiting access to food, health, and education benefits, and in general creating an environment in which there is a constant threat of document verification and subsequent deportation. While the number of states enacting these multiissue laws has been limited, 2011 brought a particular surge - five states enacted omnibus legislation, while some eight states had similar legislation pending by year's end. The

\footnotetext{
${ }^{2}$ All statistics in this section are obtained from the National Conference of State Legislatures website, www.ncsl.org. All categories listed at www.ncsl.org are included in Table 1.1 with the exception of 'resolutions,' due to the fact that these laws tend to have no relation to a detrimental effect on immigrants. Budget laws are included in the 'Miscellaneous' category.
} 
Appendix lists the fourteen states having already passed omnibus laws, accompanied by a summary of the issues addressed in each law.

\section{Theoretical impact}

Understanding the theoretical effect of the implementation of state omnibus immigration laws on the population and employment numbers of undocumented immigrants involves a simple cost and benefit analysis. Assuming that each individual has some benefit and some cost corresponding to living (working) in a particular geographic location, state immigration laws result in an increased cost burdening each undocumented individual. ${ }^{3}$ The cost could take various forms: an evasion cost, a discrimination cost, or a psychological cost, just to name a few. If all individuals only accept positive payoffs, a first indicator of an individual's (re)location decision is if the associated benefit is still higher than the now greater cost. However, even if the total payoff remains positive, the relevant indicator is the comparison of that total payoff to all other available payoffs, those potentially realized by migration to another US state or migration to another country. Clearly, if other possible payoffs do not differ greatly from the payoff of the immigrant's state of residence before the implementation of omnibus immigration law, the extra cost imposed by implementation could change an individual's payoff-maximizing location, ultimately causing out-migration. However, if a particular location gives a preimplementation payoff much higher than all other options, a payoff-maximizing

\footnotetext{
${ }^{3}$ Although a substantial literature examines individuals' decisions to migrate, many times detailing the specific associated costs and benefits at great length, I choose to lump these into one generalized cost and benefit for each individual for reasons of simplifying the demonstration of the theoretical impact of immigration law.
} 
undocumented individual could rationally choose to stay in a state even after implementation of immigration law.

An additional potential impact of immigration law is the effect implementation may have on employers, outlined in Raphael and Ronconi (2009). Most omnibus laws include some measure related to employment status verification, punishing employers caught hiring undocumented workers. If this is the case, employers now bear a higher cost related to hiring this segment of the population, translating into a lower payoff for undocumented individuals as the probability of obtaining the benefit associated with employment decreases.

Whether or not this hypothesized outflow of immigrants in turn incentivizes a response from the native or documented immigrant population is a more complicated question, due to the fact that the answer depends on the substitutability of these groups, a topic that numerous studies continue to examine. As much of the undocumented population is relatively low-skilled, these studies generally focus on the production substitutability of low-skilled natives (or low-skilled, foreign-born citizens) for immigrants. Ottoviano and Peri (2012) estimates that natives and immigrants of similar skill in the U.S. are imperfect substitutes, while Borjas et al. (2008) estimates an infinite substitution, pointing to the perfect substitutability of these demographic groups. After finding imperfect substitution among similarly-skilled natives and immigrants, Card (2009) points out that those most affected in terms of employment by the arrival of immigrants are those immigrants who had previously established residence in the area under examination.

As the issue of substitutability continues to be debated, various additional studies empirically analyze the related topic of whether immigrant inflows are associated with 
native outflows (the partner research question to the present study), often focusing on how these flows may in turn affect workers' wages. Studies such as Filer (1992), Frey (1995), Borjas et al. (1997), and Borjas (2006) contend that immigration inflows do lead to native outflows; on the other hand, others including Wright et al. (1997), Card (2001), Kritz and Gurak (2001), and Peri and Sparber (2008) argue that native outflows, if present, do not stem from immigration inflows.

One of the central economic arguments stated by immigration law supporters, that of 'immigrants take natives' jobs,' follows directly from the findings of the former group of studies. In the current context of state immigration legislation, this reasoning implies that if immigrants in fact emigrate to another state (or country), natives will fill those jobs that were previously 'taken.' In the words of omnibus immigration law author Kris Kobach, this implication taken literally translates into 'if you want to create a job for a US citizen tomorrow, deport an illegal alien today.' ${ }^{4}$ Logically, the possible inflow of natives could manifest itself in one of two fashions: 1) population and worker inflows of natives moving from other states looking to replace the immigrant-vacated positions, or 2) worker inflows from the native population already present in the respective state (previously either unemployed or not in the labor force). The former possibility alters both population and employment numbers; the latter possibility does not appear as a change in the native population, but clearly surfaces as a change in the employment numbers. Following this line of thinking, this expected native inflow should be especially notable in times of high

\footnotetext{
${ }^{4}$ I first encountered this commentary from the Kansas Secretary of State, who doubled as immigration advisor to U.S. presidential candidate Mitt Romney, as reported by Univision's Noticiero Edición Nocturna (Nightly News Edition). Video of Kobach's related comments, as part of the Conservative Political Action Conference (CPAC), is readily accessible on the Internet.
} 
unemployment, such as those experienced during much of the period in focus. ${ }^{5}$ Furthermore, since the geographical unit under examination is at the state level, the empirical setup is particularly conducive to observing this native inflow. Borjas (2006) points out that larger outflow (inflow) effects as a native response to immigration are found 'the easier that natives find it to "vote with their feet”.' If inflows are indeed stemming mostly from within-state individuals previously not employed, this study obtains the largest possible inflow observable since there is absolutely zero cost to moving. In fact, a native inflow into employment does not even necessarily require 'voting with their feet.' Considering these conditions stacking the deck in favor of observing native inflows, if these inflows do not accompany immigrant outflows, the economic argument supporting stricter immigration policy quickly loses any traction it may have had.

\section{Data and Empirical Strategy}

In detailing the different possible migratory manifestations, I examine the data from the CPS both in terms of population and employment for all individuals ages 20 to 60 . Monthly microdata cover six years, allowing for analysis of the period August 2005 to September 2011. I classify the population and employment data according to 52 demographic groups, forming an essential part of my empirical strategy. Demographic indicators include citizenship/nativity, race/ethnicity, and education. Citizenship/nativity divides the population into four groups: U.S. citizen/born in U.S., U.S. naturalized citizen/foreign-born, noncitizen/foreign-born, and noncitizen/foreign-born/arrived to U.S.

\footnotetext{
${ }^{5}$ Unemployment rates calculated from the CPS reflect an average of nearly 7\% unemployment in states at respective times of omnibus law implementation. While high compared temporally to adjacent periods within states, the average is below the national unemployment rate average of $7.7 \%$ for the same monthyear combinations of implementation.
} 
after 1982. While the first three groups are clearly mutually exclusive, noncitizen/foreignborn and noncitizen/foreign-born/arrived to U.S. after 1982 are not, intentionally allowing for what I call 'demographic narrowing.' Demographic narrowing is my main strategy for pinpointing and comparing the demographic groups most likely (not) affected by the implementation of immigration law; it allows for narrowing the focus from a larger group, such as noncitizen/foreign-born, to a smaller portion of the larger group, such as those foreign-born noncitizens arriving to the U.S. after 1982. The necessity arises because the CPS and other available data sources do not specify legal or illegal status of the noncitizen population. However, by use of undocumented population estimates and a well-constructed demographic narrowing process, I can confidently identify those groups that are theoretically most affected. ${ }^{6}$ As an example, the specification of noncitizen/foreignborn/arrived to U.S. after 1982 allows for pinpointing the narrowest group most likely targeted by the laws in terms of citizenship/nativity status. The group should have more members of illegal status than the noncitizen/foreign-born because of the Immigration Act of 1986, which legalized the residency of any immigrant present in the U.S. prior to 1982. While the obvious presence of many legal residents in the 'noncitizen/foreign-born/arrived to U.S. after 1982' category leaves this specification far from a perfect identification of the undocumented population, the narrowing achieves a second-best approximation of the targeted population. ${ }^{7}$

\footnotetext{
${ }^{6}$ Passel and Cohn (2009) outlines these estimates, signaling for example, that of the 11.9 million undocumented immigrants in the U.S., $76 \%$ are Hispanic.

${ }^{7}$ Achieving the 'best approximation,' i.e. exact identification of the undocumented population, through surveys such as the CPS may actually not be ideal for this paper's purposes. If survey participants were explicitly asked about legal residency status, many undocumented residents would likely not willfully respond to the survey, creating an even stronger tendency toward non-response from this demographic than that which already exists.
} 
Race/ethnicity separates individuals into four groups as well: white/non-Hispanic, black/non-Hispanic, Asian/non-Hispanic, and Hispanic. The specific, perhaps unorthodox classification marks an intentional effort to avoid potential ambiguity, given the manner in which the CPS race and ethnicity questions are formulated. The survey classifies race by the options of 'white only,' 'black only,' 'American Indian only,' 'Asian only,' 'Hawaiian only,' or any combinations of the above; Hispanic or non-Hispanic status is determined in a separate question. The problem arises from the fact that these two classifications exhibit overlap; nearly all individuals who identify themselves as Hispanic also identify race as white or black, with a lower number identifying the other race categories. If this overlap were not accounted for and individuals were in turn classified simply as white, black, or Hispanic, a majority of Hispanics would be double-counted, causing an enormous problem of bias. Education simply classifies people as low-skilled or high-skilled, completion of high school marking the upper limit of the low-skilled category. ${ }^{8}$

For each of the 52 demographic groups, I specify an econometric model of the following form:

$$
Y_{i t}=c+\alpha_{i}+\gamma_{t}+\beta_{1} \text { Treat }_{i t}+\beta_{2} \text { Post }_{i t}+\beta_{3} \text { Treat }_{i t} \text { Post }_{i t}+\varepsilon_{i t}
$$

The fixed effects/difference-in-differences specification employs dummy variables for all states and month-year combinations, represented by $\alpha_{i}$ and $\gamma_{t}$, respectively. The placeholder $Y_{i t}$ is for the variable of interest, either population or employment of state $i$ in month-year $t$ for each demographic group. Treat ${ }_{i t}$ is a dummy variable taking a value of one for all $t$ if state $i$ receives treatment (omnibus immigration law implementation) at any

\footnotetext{
${ }^{8}$ This education dividing line is selected based on results from Card (2009) finding that 'high school equivalent' and 'college equivalent' workers are imperfect substitutes.
} 
point in the 2005 to 2011 period. Post ${ }_{i t}$ is a dummy variable taking a value of one if for state $i$, period $t$ is post-treatment. In turn, Treat ${ }_{i t}$ Post $_{i t}$ takes a value of one only when state $i$ actually receives treatment in month-year $t . \alpha_{i}$ controls for any observable or unobservable systematic differences across states, while $\gamma_{t}$ controls for any differences over time that may affect all states' outcomes, such as changes to federal immigration law. ${ }^{9}$ $\beta_{3}$ is the coefficient of interest, the difference-in-differences estimate representing the change in the lefthand-side variable associated with omnibus immigration law implementation. ${ }^{10}$ I examine population and employment of the demographic groups in terms of both proportions and levels. First, by expressing population (employment) as logarithms, I use the specified equation to estimate the percentage change in the variable of interest associated with state immigration law implementation. Second, I use the logarithm of the demographic group to state total ratio for each variable of interest to estimate percentage changes in the composition of population (employment) associated with the implementation of state immigration law. ${ }^{11}$

\footnotetext{
${ }^{9}$ A potential concern may be that part of the period under examination is spanned by an economic crisis in the U.S.. However, any effects from the economic crisis are controlled for by the inclusion of the monthyear combination time fixed effects; furthermore, if individual states suffered more or less than other states due to the financial crisis, this is controlled for by the individual state fixed effects.

${ }^{10}$ While the difference-in-difference-in-differences (DDD) method is an attractive alternative to differencein-differences (DD), DDD does not improve on DD in this study's case. The inclusion in DDD of the within-state control group is problematic due to the fact that I expect some effect on the population and employment of those 'non-immigrant' demographic groups in states receiving treatment. If these groups were to form a within-state control group as part of the DDD estimator, the measured effect of the state immigration legislation would obtain an upward bias. By employing DD, I avoid this potential bias and therefore am able to separate out the effects on the various demographic groups and their respective responses to implementation of the immigration laws.
}

${ }^{11}$ For each ratio, the population (employment) of the specific demographic group being examined serves as the numerator, while the state total serves as the denominator. 
For each set of regressions, I define two different control groups: 1) neighboring states of each respective state receiving treatment and 2) all U.S. states. While use of the first control group permits a potential bias due to the possibility of outflows (inflows) affecting mainly neighboring states, this possibility seems not to have manifested itself. The data show only slight changes in neighboring states' population and employment after immigration legislation. Furthermore, this is to be expected - the immigrant outflows may be large especially in terms of percentages, however the associated levels result in only small changes for receiving states when spread out over many states of relocation. Nonetheless, the inclusion of the second control group, all U.S. states, allows for a simple comparison, theoretically minimizing any bias that may exist through use of neighboring states in the regional control group. I exclude states from control group consideration if treatment is received during or prior to the respective legislation implementation period under examination. For the baseline regressions, six months on either side of the legislation's implementation date form the legislation implementation period and enter as observations into the regressions, allowing for a certain amount of flexibility in capturing any population and employment changes that result from omnibus legislation but may not be captured by only examining the months immediately before and after implementation.

I confirm the presence of a common trend with the results of t-tests reported in Tables 1.2 and 1.3. For each of the six months in the period prior to implementation, a ttest is performed comparing the portions of population from the treatment group with those of the control group made up by each of four demographic groups of central importance: Noncitizen/foreign-born/arrived to U.S. after 1982/Hispanic/low-skilled, native/Hispanic/low-skilled, native/white/non-Hispanic/low-skilled, and native/black/ 
non-Hispanic/low-skilled. ${ }^{12}$ While small differences in means are certainly present, these differences are minimal and not statistically significant at the $5 \%$ level, evidenced by all tstatistics below an absolute value of 0.70 in Table 1.2 for the regional control group. Use of the alternative national control group results in no statistically significant differences at the $5 \%$ level, and all t-statistics are below an absolute value of 0.82 . Given the evident presence of the common trend, I thereby verify the central identification assumption and validate the difference-in-differences estimates.

The nature of the state omnibus immigration legislation process presents an additional problem in relation to defining the point of separation for pre- and posttreatment, due to the varying lapse of time between law enactment and implementation as well as various challenges to the legality of the omnibus laws (outlined in the Appendix). I choose the established implementation date (labelled as "effective" in the Appendix) as the separation between pre- and post-treatment. Legislation establishes this date at least by the time it is enacted, therefore giving a period on average of six months for the population to adjust their employment/residency plans according to their expectations. Therefore, even in states such as Oklahoma where legal challenges postponed complete implementation of the immigration legislation, these expectations and accordingly adjusted plans presumably still have an effect as far as population and employment movements resulting from the omnibus laws.

\footnotetext{
${ }^{12}$ Assuming an implementation month of $\tau, \tau-1$ represents one month prior to implementation, $\tau-2$ represents two months prior to implementation, and so on. The results of t-tests for portions of employment are not reported, but are available upon request from the author; these results are highly similar to those of population.
} 
Two additional aspects of my empirical strategy address specific critiques as to the preciseness of difference-in-differences estimation expressed in the recent literature. First, by aggregating population and employment data at the state level rather than including individual-level observations from the CPS in the specified regressions, I avoid the problem of common group errors presented by multilevel data emphasized in Donald and Lang (2007). In addition, I follow the recommendations of Bertrand et al. (2004) in calculating Huber-White robust standard errors clustered by state for inference purposes, allowing for unrestricted error correlations across observations within states and thereby accounting for the serious concern of serial correlation in the data.

\section{Results and Discussion}

Of the 52 demographic groups identified by the three indicators in this study, Passel and Cohn's figures help point to the group of 'noncitizen/foreign-born/arrived to US after 1982/Hispanic/low-skilled' as containing the highest percentage of undocumented population, and in turn theoretically most likely to be negatively affected in terms of population and/or employment by state immigration law implementation. In documenting the migratory outflows associated with the state omnibus laws, I include estimates in Tables 1.4 and 1.5 for this narrowest demographic, as well as the following broader demographic divisions, in descending order of expected percentage of undocumented population: 'noncitizen/foreign-born/arrived to U.S. after 1982/Hispanic,' 'noncitizen/foreign-born/arrived to U.S. after 1982,' 'noncitizen/foreign-born/ Hispanic/low-skilled,' 'noncitizen/foreign-born/Hispanic,' and 'noncitizen/foreign-born. ${ }^{13}$

\footnotetext{
${ }^{13}$ All estimates related to employment numbers are available in the Appendix.
} 
In attempting to gauge the impact of the laws and any subsequent immigrant movements on the behavior of other demographic groups, I focus on several segments of the population with no undocumented individuals that economic theory has presented as possible substitutes for the already-mentioned groups with the highest percentages of undocumented immigrants. ${ }^{14}$ Borjas et al. (2011) argues that similarly-skilled immigrants and natives are perfect substitutes according to U.S. labor evidence, while Card (2009) suggests that if immigrants and natives are imperfect substitutes, additional immigrants would be the group designated as nearest-to-perfect substitute for immigrants already present in the U.S.. I take both findings to the data in the context of my study, documenting the following demographic groups' population changes in Table 1.6 and employment changes in Table 1.7: 'native/Hispanic/low-skilled,' 'native/white, non-Hispanic/lowskilled,' and 'native/black, non-Hispanic/low-skilled' in order to capture any nativeimmigrant substitutability, and 'naturalized citizen/foreign-born/Hispanic/low-skilled' in order to capture any immigrant-immigrant substitutability. To further shed light on the population and employment movement responses to immigration law, I also include the total change of state population (employment) associated with implementation of the legislation.

\section{Impacts on Population}

Figure 1.1 gives an idea as to the migratory outflows of immigrants in terms of population during the implementation period of omnibus immigration legislation. However, although an average decline in the examined demographic's proportion is

\footnotetext{
${ }^{14}$ By substitutes, I refer to the terminology used in labor economics, which identifies groups that tend to substitute or complement one another, classified according to characteristics such as citizenship status, race/ethnicity, and skill level.
} 
observed in the six months after implementation, this decrease is evidence from raw data not yet taking advantage of the treatment and control difference-in-differences methodology necessary for obtaining the desired estimates of interest. Panel A of Table 4 shows the impact on population associated with the immigration law implementation for those demographic groups having the highest percentages of undocumented immigrants. I estimate that the implementation of omnibus laws is associated with a $24.41 \%$ decrease in the low-skilled, Hispanic, noncitizen population arriving post-1982 to the US, when comparing states receiving treatment to those geographic neighbors not receiving treatment. ${ }^{15}$ While this group is the group weighted most heavily with undocumented immigrants, even the group of foreign-born noncitizens, which certainly has a much lower percentage of undocumented population, experiences a decline of 9.93\% associated with the law implementation. When the alternative control group of all US states is used, minimizing the possible bias created by expected inflows to neighboring states, the magnitude of these effects are only slightly attenuated, $19.02 \%$ and $8.22 \%$, respectively. Interestingly, since the total population also experiences an associated decrease, albeit small in magnitude, the proportion changes detailed in Panel B of Table 1.4 are smaller in percentage terms than their Panel A level counterparts. All estimates in Table 1.4 are statistically significant at the $5 \%$ level, indeed pointing to a strong outflow of (undocumented) immigrant population in response to the state omnibus immigration laws.

Estimates in Table 1.6 outline the impact on those groups identified by economic theory as possible substitutes for immigrant groups, therefore leading to an expectation of

\footnotetext{
${ }^{15}$ The estimates reported approximate percentage changes, as population (employment) is expressed in logarithmic form and estimates are then multiplied by a factor of 100 .
} 
inflows associated with immigrant outflows. However, of the four groups examined, only the native, black/non-Hispanic, low-skilled group has an associated population increase, magnitude of $6.51 \%$. Both native and naturalized citizen groups of low-skilled Hispanics actually reflect a clear population decline, losing $11.57 \%$ and $4.70 \%$ respectively. While these estimates are not as significant as those in Table 1.4, the evidence does point to a lack of 'replacement' population for the outflow of immigrants. This fact is confirmed by the slightly negative estimates for total state population, $-0.61 \%$ when employing the regional control group and $-0.45 \%$ when including all U.S. states. The negative impact on groups not targeted by omnibus legislation is a noteworthy finding, likely due to one or both of two possibilities. First, low-skilled, Hispanic legal immigrants (or natives) may be suffering a type of workplace discrimination. As some of the omnibus legislation affects employers directly, some employers may be more reluctant to hire any worker who fits whatever profile the employer may associate with undocumented workers. On the other hand, this result could reflect that individuals in this demographic anticipate discriminatory treatment as a result of the immigration laws and therefore relocate, or simply decide not to live and work in a place where laws could unfortunately lead to instances of racial profiling. An additional consideration mentioned in Raphael and Ronconi (2009) is the fact that some immigrant families are made up of both undocumented and documented immigrants, leading to the out-migration of all family members when the undocumented are targeted by immigration law. In either case, these figures point to a central complaint of opponents of the state omnibus immigration laws - that on top of the negative effect on undocumented immigrants, the legislation most likely has an unintended, adverse effect on certain segments of the legal, documented immigrant population. 


\section{Impacts on Employment}

Figure 1.2 outlines the migratory outflows of immigrants in terms of employment during the implementation period of omnibus immigration legislation. Panel A of Table 1.5 displays estimates for the immigrant demographic groups' that reflect a decline in employment associated with implementation of the immigration laws. Estimates range from $-20.73 \%$ to $-10.62 \%$, evidencing a notable fall in employment, however generally slightly smaller in magnitude than that of these same groups' population change using the regional control group. In line with the population estimates, use of all U.S. states as the control group results in employment estimates of a lower magnitude for the examined immigrant demographic groups, with the largest effect being $-16.32 \%$ for the noncitizen, Hispanic, low-skilled, arrived to U.S. post-1982 group. Once again, estimates for these groups are statistically significant, with only that of noncitizen/Hispanic not being significant at least the $10 \%$ level, the majority significant at the $5 \%$ level.

In examining the possible substitute groups listed in Table 1.7, the estimates for employment highlight two important trends. First, the immigration legislation again appears to adversely affect segments of the population other than those groups with high percentages of undocumented immigrants, even groups that are hypothesized to be substitutes for the immigrant groups. Panel A of Table 1.7 shows a $10.89 \%$ and a $14.60 \%$

decrease in employment level, respectively, for native, low-skilled Hispanics and naturalized citizen, low-skilled Hispanics when using regional control groups. While these estimates do vary when changing to all U.S. states as the control group of choice, they remain clearly negative. The same two possibilities hypothesized above in the discussion of the population estimates provide the clearest explanation as to why this result obtains. 
Second, for the group of native, low-skilled blacks (non-Hispanics), there does appear to be some substitute or replacement effect in terms of employment. Estimates of $7.79 \%$ and $10.68 \%$ for the regional and overall controls, respectively, signal a positive impact on employment for this demographic. However, this rise in employment is not shared by native, low-skilled whites (non-Hispanics), and the estimates throughout Table 1.7 are plagued by lower significance compared with those of Table 1.5. In fact, the overall gauge of the impact on employment, the estimate for the state total, is slightly negative using both control groups, just as in the case of population.

\section{Checks on Initial Estimates}

While the above estimates paint a relatively clear picture of the migratory responses to state immigration law implementation, the skeptical reader may raise concerns as to several issues worth addressing, including the robustness, sensitivity, and reliability of the estimates.

\section{Robustness and Sensitivity}

To this point, results obtain through the examination of a one-year implementation period, including all implementing states according to their effective date even if the actual implementation faces delays due to legal challenges. In order to first check the robustness of these results, I explore what changes may occur if Oklahoma's effective date of November 2007 is excluded from the treatment group. As detailed in the Appendix, the courts initially prevented a major section of Oklahoma's omnibus law from taking effect. As previously explained, I include Oklahoma in the initial treatment group based on those expectations that accompany the effective date, even if not all sections of the omnibus legislation ultimately take effect on this date. However, examining a second set of 
regressions excluding Oklahoma allows for a simple check of whether the initial results are unduly influenced by Oklahoma's inclusion.

Tables 1.8 and 1.9 clearly show that the baseline results are robust to the exclusion of Oklahoma's effective date from the treatment group. The coefficients displayed in Table 1.8 show all negative estimates larger in magnitude than those of Tables 1.4 and 1.5. This makes logical sense, since some immigrants waiting until the last moment (the expected effective date) to out-migrate may have opted to remain in Oklahoma when the employment section of this particular legislation did not take immediate effect, resulting in a smaller outflow than that which would have otherwise occurred. As in Table 1.4, all population estimates are significant at the $5 \%$ level, however unlike Table 1.5 , all employment estimates are now significant at the 5\% level, as well. Comparing Table 1.9 with the corresponding Tables 1.6 and 1.7, population estimates in Table 1.9 are larger in magnitude (both positive and negative), with no changes in sign from the previous tables. For example, using the regional control results in a $14.53 \%$ decrease for native, low-skilled Hispanics and an $11.44 \%$ increase for native, low-skilled blacks (non-Hispanics). On the other hand, Table 1.9 lists somewhat attenuated employment estimates for the regional control, all coefficients consistent in sign with the exception of native, low-skilled whites (non-Hispanics). Estimates for the change in total population (employment) are consistent with the baseline regressions, ranging from $-0.36 \%$ to $-0.57 \%$, although just as before the estimates for 'natives' are not as significant as those for 'immigrants.'

Turning to the issue of timing, I examine how sensitive the results are to changing the length of the implementation period. Tables 1.10 and 1.11 display the estimates of interest given a total implementation period of 6 months in Panel A, while estimates from 
total implementation periods of 2 years are in Panel B. ${ }^{16}$ Six-month population and employment coefficients from Table 1.10 are extremely similar in magnitude and significance to those of Tables 1.4 and 1.5, however two-year coefficients are less significant and attenuated in magnitude, employment estimates for foreign-born noncitizens turning slightly positive. While the magnitude and significance vary for six-month estimates in Table 1.11, all signs remain consistent with those from Tables 1.6 and 1.7 except for population estimates related to naturalized, low-skilled Hispanics. Two-year coefficients paint the same picture as the corresponding six-month estimates, however there is additional sign disagreement in the estimates for native, low-skilled blacks (nonHispanics). In summary, while the baseline results do show some sensitivity to altering the length of the examined implementation period, both the six-month and two-year samples confirm general trends and resulting conclusions, the six-month results even matching those of the baseline regressions in magnitude and significance.

\section{Endogeneity Bias?}

An important concern as to the reliability of the difference-in-differences estimates is the issue of endogeneity. The implementation of state omnibus immigration legislation must be exogenous in order for the estimates to capture the true effect on population and employment, free from any endogeneity bias. Raphael and Ronconi (2009) addresses this concern in detail, pointing to evidence from the Pew Hispanic Center's National Survey of Latinos (NSL) as discounting the possibility that the laws' implementation are actually driven by other changes, leading to a problem of endogeneity. They examine both the

\footnotetext{
${ }^{16}$ These are equivalent to a pre- and post-implementation period of 3 months each and 1 year each, respectively, compared with the baseline regressions using a pre- and post-implementation period of 6 months each.
} 
possibility of higher levels of discriminatory attitudes as well as that of changes in levels of discriminatory attitudes leading to immigration law implementation; however, through the data provided by the NSL both of these possible arguments of endogeneity are discarded.

An additional cause of endogeneity could arise from the simple case of the (undocumented) immigrant population increasing in size to a certain percentage of a state's total population, arriving to a tipping point which in turn triggers the beginnings of the process of immigration law enactment and implementation. ${ }^{17}$ It is common knowledge that a network effect indeed exists in the migration decision, resulting in regions with high percentages of immigrants tending to draw more immigrants. ${ }^{18}$ However, while the network effect could point to a tipping point eventually obtaining, its existence is clearly not sufficient for the tipping point to obtain. In fact, evidence from Passel and Cohn (2009) refutes the tipping point theory, citing the fact that the 'undocumented immigrant population grew rapidly from 1991 to 2006 but has since stabilized.' Since this stabilization occurred prior to the influx of state omnibus immigration legislation, any hesitation as to endogeneity is somewhat pacified. However, to further examine this possibility, especially given the fact that a lag between stabilization and immigration legislation implementation could occur, I again examine the narrowest demographic - noncitizen/foreign-born/arrived to U.S. after 1982/Hispanic/ low-skilled - specifically, I examine the exact proportion of

\footnotetext{
${ }^{17}$ One might imagine a different sort of tipping point, in which the (undocumented) immigrant population increases in size until it gains sufficient political power to in turn influence immigration policy away from omnibus legislation. However, I examine the tipping point in terms of triggering omnibus legislation due to the fact that many immigrants (clearly, undocumented immigrants) do not have voting rights in the US and therefore have minimal political clout.

${ }^{18}$ For a sample of the literature examining network effects and the migration decision, see McKenzie and Rapoport (2007).
} 
each state's population made up by this group, measured one year before implementation of omnibus legislation. This date serves as an approximate marker for when the legislative process of proposing and discussing new legislation actually takes place. Figure 1.3 (Figure 1.4) shows the corresponding proportions of population (employment) for each state implementing omnibus law, along with the proportions of the same demographic group for all other states not implementing immigration law during the same period. Table 1.12 lists the rank of each proportion compared to all other state counterparts for the same time period.

Confirming the evidence from Passel and Cohn (2009), there does not appear to be a tipping point that sets in motion the adoption of omnibus immigration legislation, as the proportions exhibit a wide variety in their rankings. Using regional states as a comparison group, Georgia, Utah, and Arizona each rank third in the population measure among their respective regions, however Missouri, Oklahoma, and South Carolina rank in the bottom half of their regions, South Carolina ranking only ninth out of eleven. Regional employment rankings reflect much the same, although Nebraska joins the group of lowranking states. Switching to all US states as a comparison group, Arizona and Utah both experience relatively high overall proportions of population (employment) of the targeted demographic group one year before implementation, however other implementing states can be classified as having relatively moderate or low proportions. Missouri, South Carolina, and Oklahoma certainly qualify as having relatively low proportions of both population and employment, with overall population rankings of 27, 26, and 27, respectively. Additionally, Nebraska's overall rank of 30 for employment is the lowest statistic among the legislation-implementing states. 
Table 1.13 outlines a further test providing evidence against the tipping point theory. Panel A displays the results of regressing the implementation of state immigration law on the one-year lag of the narrowest demographic proportion detailed above, while Panel B shows the results of regressing the implementation of state immigration law on the one-year lag of the change in the narrowest demographic proportion. Any positive relationship between this (change in) proportion and subsequent immigration law implementation should clearly appear as positive, significant coefficients in the regression results. However, coefficients are insignificant and imprecisely estimated, each respective 95\% confidence interval including the possibility of both a negative and a positive relationship between the two respective variables. Furthermore, if these estimates were significant and precisely estimated, the association between the variables would remain small in scale given the interpretation of the estimates. The highest coefficient in magnitude in Panel A signals that even a one-unit increase in the narrowest demographic proportion (not possible since the independent variable is a proportion measured between zero and one) is only associated with a $51 \%$ increase in the probability that a state implements omnibus immigration legislation. Correspondingly, the coefficient of highest magnitude in Panel B signals that a one-unit increase in the change of the narrowest demographic proportion is associated with a $24 \%$ decrease in the probability that a state implements omnibus legislation.

Together with the evidence provided by Raphael and Ronconi (2009) and Passel and Cohn (2009), these trends (or lack thereof) strongly signal that the tipping point theory is not in line with empirical fact. The identified empirical strategy and resulting estimates are indeed free from worries of endogeneity, thereby contributing a reliable estimation of 
the effect of immigration legislation on population and employment of the examined demographic groups.

\section{Conclusion}

The estimated impact of the implementation of state omnibus immigration legislation on total state population and employment is consistently slightly negative, ranging from $-0.45 \%$ to $-0.61 \%$ for population and $-0.52 \%$ to $-0.77 \%$ for employment. While somewhat imprecisely measured, these estimates' 95\% confidence intervals indicate that they are almost certainly negative in sign, leaving only a relatively small possibility of any positive relationship. With the exception of native, low-skilled blacks (non-Hispanics), there appears to be no substitution in terms of inflows from those demographic groups predicted by theory as being probable substitutes for the undocumented immigrant population. Some of these groups actually experience an adverse impact on population and employment associated with implementation of the immigration legislation. This adverse impact is in addition to the highly statistically significant immigrant outflows of up to nearly a quarter of the corresponding population, as large as $24.41 \%$ in terms of population and $20.71 \%$ in terms of employment for the examined 'immigrant' demographic groups.

These findings point to the fact that omnibus legislation not only has a negative effect on the undocumented population, but it also unintentionally harms a much broader segment of the population. With substitution inflows on a smaller scale than the corresponding outflows, the empirical evidence clearly does not support the proimmigration law partner statements of 'immigrants take natives' jobs' and 'if you want to create a job for a U.S. citizen tomorrow, deport an illegal alien today.' This combination of adverse effects on population and employment at three demographic levels - the 
undocumented population, the affected 'substitute' groups that actually do not substitute, and the total state populations - deserves careful consideration from not only economists, but from the wider policymaking community as well. As increasing the welfare related to states' population, especially in terms of employment, is the very reason posited by policymakers as justification for immigration law implementation, this trifecta of adverse effects leaves little doubt that state omnibus immigration legislation is not completing its stated mission.

\section{Appendix}

\section{Georgia SB-529}

- Enacted 17 April 2006; effective 1 July 2007

- Work authorization, penalty for human trafficking, local immigration law enforcement, determination of legal status when arrested, eliminates public benefits

2. Oklahoma HB-1804

- Enacted 8 May 2007; effective 1 November 2007

- Work authorization, identification/driver's licenses, local immigration law enforcement, felony to harbor/transport unauthorized immigrants, eliminates public benefits

- Legal Challenge - Employment section barred from taking effect, except for E-Verify system

3. Missouri HB-1549

• Enacted 7 July 2008; effective 28 August 2008 (1 January 2009) 
- Work authorization, identification/driver's licenses, local immigration law enforcement, eliminates public benefits

4. South Carolina HB-4400

- Enacted 4 June 2008; effective 4 June 2008 (except for work authorization effective 1 January 2009)

- Work authorization, identification/driver's licenses, local immigration law enforcement, eliminates public benefits, education restrictions

5. Utah SB-81

• Enacted 13 March 2008; effective 1 July 2009

- Work authorization, identification/driver's licenses, local immigration law enforcement, eliminates public benefits

6. Georgia HB-2

• Enacted 11 May 2009; effective 1 January 2010

- Work authorization, determination of legal status when arrested, eliminates public benefits

7. Missouri HB-390

• Enacted 7 July 2009; effective 7 July 2009

-Work authorization, public benefits, education restrictions

8. Nebraska LB-403

• Enacted 8 April 2009; effective 1 October 2009

-Work authorization, eliminates public benefits

9. Arizona SB-1070, HB-2162

- Enacted 23 April 2010; effective 29 July 10 
- Work authorization, local immigration law enforcement, warrantless arrest, citizens can sue agencies for nocompliance, document-carrying policy

- Legal challenge by U.S. Department of Justice

- Three provisions preliminarily barred from taking effect

- Pending appeal by Arizona governor Jan Brewer

10. Utah H116, H466, H469, H497

- Enacted 15 March 2011; effective

- Work authorization, immigrant integration, proposed temporary worker program by 2013

- Legal challenge by Utah Coalition of La Raza

- H497 temporarily restrained

11. Georgia HB-87

- Enacted 13 May 2011; effective 1 July 2011 (except public benefits - effective 1 July 2012)

- Work authorization, local immigration law enforcement, eliminates public benefits

- Legal challenge by Georgia Latino Alliance for Human Rights et al.

- Two provisions enjoined preliminarily

12. Indiana SB-590

• Enacted 10 May 2011; effective 1 July 2011

- Work authorization, eliminates public benefits, requests reimbursement of 'immigrant cost' from federal government

- Legal challenge by Ingrid Buquer, et al. 
- Two provisions enjoined preliminarily

13. Alabama HB-56

- Enacted 9 June 2011; effective 1 September 2011

- Work authorization, local immigration law enforcement, felony to harbor/rent to unauthorized immigrants, document-carrying policy, questioning of legal status in public schools

- Legal challenge by US Department of Justice

- Two provisions barred from taking effect 14 October 2011

14. South Carolina S-20

- Enacted 27 June 2011; effective 1 January 2012

- Work authorization, identification/driver's licenses, eliminates public benefits, felony to harbor/rent to unauthorized immigrants

- Legal challenge by US Department of Justice

- Pending challenge filed 31 October 2011

Table 1.1. Enacted state immigration laws: 2005 to 2011

\begin{tabular}{llllllll}
\hline Issue & 2005 & 2006 & 2007 & 2008 & 2009 & 2010 & 2011 \\
\hline Education & 3 & 3 & 22 & 12 & 27 & 17 & 20 \\
Employment & 5 & 14 & 29 & 19 & 21 & 27 & 27 \\
Health & 0 & 0 & 14 & 11 & 28 & 17 & 23 \\
Human Trafficking & 9 & 13 & 18 & 5 & 16 & 8 & 5 \\
ID/Driver's License & 10 & 6 & 40 & 32 & 46 & 26 & 27 \\
Law Enforcement & 5 & 8 & 16 & 12 & 16 & 37 & 39
\end{tabular}




\begin{tabular}{llllllll} 
Miscellaneous & 7 & 11 & 17 & 38 & 46 & 69 & 31 \\
Omnibus & 0 & 1 & 1 & 3 & 3 & 2 & 6 \\
Public Benefits & 5 & 10 & 33 & 9 & 15 & 9 & 15 \\
Voting & 1 & 6 & 0 & 1 & 4 & 6 & 4 \\
\hline Total Passed by Legislature & 45 & 72 & 190 & 142 & 222 & 218 & 197 \\
Vetoed by Governor & 6 & 6 & 12 & 3 & 20 & 10 & 15 \\
Total Enacted & 39 & 66 & 178 & 139 & 202 & 208 & 182 \\
\hline
\end{tabular}

Table 1.2. Pre-implementation characteristics of states, regional control

\begin{tabular}{llllll}
\hline & \multicolumn{3}{c}{ Treatment group } & \multicolumn{2}{c}{ Control group (regional) } \\
Time prior to implementation & Mean & Std. error & Mean & Std. error & T-test \\
\hline Noncitizen/foreign-born/arrived to US after & 1982/Hispanic/low-skilled & \\
$\tau-6$ & 3.13 & 0.87 & 3.11 & 0.32 & -0.02 \\
$\tau-5$ & 3.48 & 0.93 & 3.08 & 0.31 & -0.40 \\
$\tau-4$ & 3.57 & 0.94 & 3.01 & 0.30 & -0.56 \\
$\tau-3$ & 3.50 & 0.95 & 3.01 & 0.31 & -0.49
\end{tabular}




\begin{tabular}{lccccc}
$\tau-2$ & 3.52 & 1.02 & 3.07 & 0.31 & -0.44 \\
$\tau-1$ & 3.30 & 0.81 & 3.11 & 0.31 & -0.19 \\
\hline Native/Hispanic/low-skilled & & & & & \\
$\tau-6$ & 2.69 & 1.52 & 2.55 & 0.47 & -0.09 \\
$\tau-5$ & 2.34 & 1.24 & 2.49 & 0.45 & 0.10 \\
$\tau-4$ & 2.55 & 1.38 & 2.43 & 0.44 & -0.08 \\
$\tau-3$ & 2.08 & 1.01 & 2.48 & 0.46 & 0.27 \\
$\tau-2$ & 2.11 & 0.96 & 2.52 & 0.48 & 0.27 \\
$\tau-1$ & 2.25 & 1.19 & 2.62 & 0.49 & 0.24 \\
\hline Native/white, non-Hispanic/low-skilled & & & \\
$\tau-6$ & 24.80 & 2.35 & 26.93 & 1.06 & 0.62 \\
$\tau-5$ & 24.39 & 2.22 & 26.80 & 1.06 & 0.70 \\
$\tau-4$ & 24.91 & 2.51 & 26.75 & 1.04 & 0.54 \\
$\tau-3$ & 26.47 & 2.49 & 26.77 & 1.03 & 0.09 \\
$\tau-2$ & 25.97 & 2.42 & 26.92 & 1.05 & 0.28 \\
$\tau-1$ & 26.11 & 2.51 & 27.02 & 1.07 & \\
\hline Native/black, non-Hispanic/low-skilled & & & 0.07 \\
$\tau-6$ & 5.31 & 2.13 & 5.47 & 0.71 & -0.01 \\
$\tau-5$ & 5.61 & 2.39 & 5.58 & 0.73 & -0.16 \\
$\tau-4$ & 5.92 & 2.41 & 5.53 & 0.72 & -0.25 \\
$\tau-3$ & 6.01 & 2.39 & 5.42 & 0.71 & -0.26 \\
$\tau-2$ & 5.99 & 2.47 & 5.37 & 0.71 & -0.19 \\
$\tau-1$ & 5.72 & 2.36 & 5.29 & 0.69 & \\
\hline
\end{tabular}

Notes: Means and standard errors express the percentage of each demographic group in the respective total state populations (population shares multiplied by 100). Time prior to implementation corresponds to an implementation month of $\tau, \tau-1$ is one month prior, $\tau-2$ is two months prior, etc.

Table 1.3. Pre-implementation characteristics of states, national control

\begin{tabular}{lccccc}
\hline & \multicolumn{3}{c}{ Treatment group } & \multicolumn{3}{c}{ Control group (national) } & \\
Time prior to implementation & Mean & Std. error & Mean & Std. error & T-test \\
\hline Noncitizen/foreign-born/arrived to US & 3.13 & 0.87 & 3.05 & 0.27 & \\
$\tau-6$ & 3.48 & 0.93 & 3.04 & 0.15 & -0.08 \\
$\tau-5$ & 3.57 & 0.94 & 2.94 & 0.15 & -0.40 \\
$\tau-4$ & 3.50 & 0.95 & 2.94 & 0.15 & -0.60 \\
$\tau-3$ & & & & & -0.54
\end{tabular}




\begin{tabular}{llllll}
$\tau-2$ & 3.52 & 1.02 & 3.00 & 0.15 & -0.46 \\
$\tau-1$ & 3.30 & 0.81 & 3.01 & 0.15 & -0.30 \\
\hline Native/Hispanic/low-skilled & & & & & \\
$\tau-6$ & 2.69 & 1.52 & 2.39 & 0.32 & -0.24 \\
$\tau-5$ & 2.34 & 1.24 & 2.39 & 0.18 & 0.01 \\
$\tau-4$ & 2.55 & 1.38 & 2.38 & 0.18 & -0.05 \\
$\tau-3$ & 2.08 & 1.01 & 2.38 & 0.19 & 0.16 \\
$\tau-2$ & 2.11 & 0.96 & 2.38 & 0.18 & 0.17 \\
$\tau-1$ & 2.25 & 1.19 & 2.45 & 0.19 & 0.36 \\
\hline Native/white, non-Hispanic/low-skilled & & & \\
$\tau-6$ & 24.80 & 2.35 & 26.32 & 0.51 & 0.44 \\
$\tau-5$ & 24.39 & 2.22 & 26.31 & 0.51 & 0.59 \\
$\tau-4$ & 24.91 & 2.51 & 26.28 & 0.51 & 0.34 \\
$\tau-3$ & 26.47 & 2.49 & 26.15 & 0.51 & -0.03 \\
$\tau-2$ & 25.97 & 2.42 & 26.18 & 0.52 & 0.06 \\
$\tau-1$ & 26.11 & 2.51 & 26.23 & 0.52 & 0.04 \\
\hline Native/black, non-Hispanic/low-skilled & & & -0.43 \\
$\tau-6$ & 5.31 & 2.13 & 4.51 & 0.27 & -0.56 \\
$\tau-5$ & 5.61 & 2.39 & 4.59 & 0.28 & -0.73 \\
$\tau-4$ & 5.92 & 2.41 & 4.55 & 0.28 & -0.81 \\
$\tau-3$ & 6.01 & 2.39 & 4.49 & 0.27 & -0.82 \\
$\tau-2$ & 5.99 & 2.47 & 4.58 & 0.28 & -0.67 \\
$\tau-1$ & 5.72 & 2.36 & 4.46 & 0.27 & \\
\hline
\end{tabular}

Notes: Means and standard errors express the percentage of each demographic group in the respective total state populations (population shares multiplied by 100). Time prior to implementation corresponds to an implementation month of $\tau, \tau-1$ is one month prior, $\tau-2$ is two months prior, etc.

Table 1.4. Difference-in-differences estimates of the impact of state omnibus immigration laws on population: immigrants

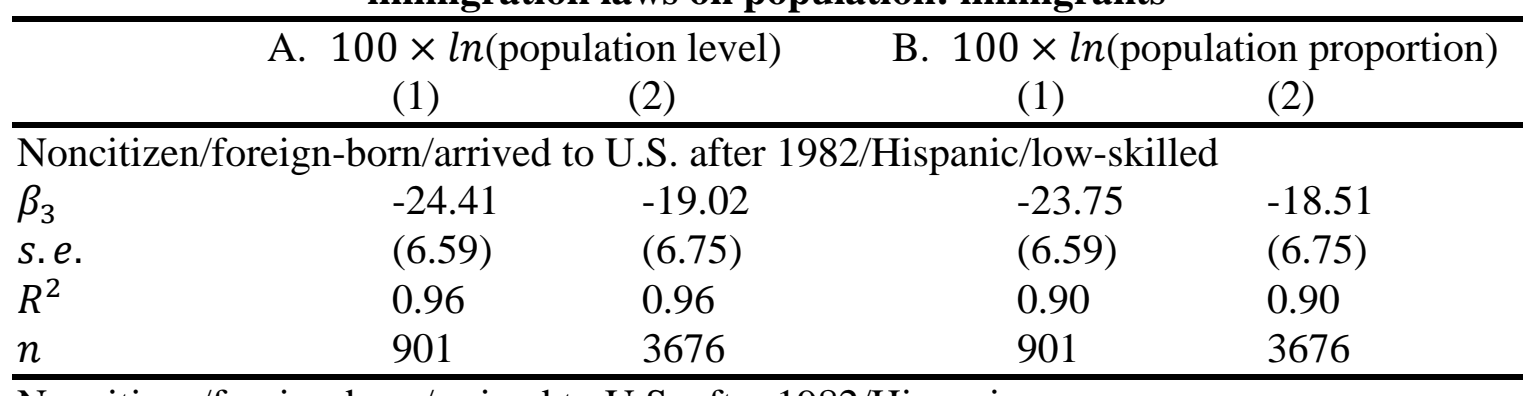

Noncitizen/foreign-born/arrived to U.S. after 1982/Hispanic 


\begin{tabular}{lllll}
$\beta_{3}$ & -22.92 & -19.27 & -22.26 & -18.79 \\
$S . e$. & $(6.59)$ & $(6.78)$ & $(6.60)$ & $(6.79)$ \\
$R^{2}$ & 0.97 & 0.97 & 0.93 & 0.91 \\
$n$ & 910 & 3763 & 910 & 3763 \\
\hline Noncitizen/foreign-born/arrived to & U.S. after 1982 & & \\
$\beta_{3}$ & -14.03 & -11.39 & -13.42 & -10.94 \\
$s . e$. & $(4.37)$ & $(4.02)$ & $(4.38)$ & $(4.08)$ \\
$R^{2}$ & 0.98 & 0.98 & 0.92 & 0.93 \\
$n$ & 924 & 3874 & 924 & 3874 \\
\hline Noncitizen/foreign-born/Hispanic/low-skilled & & \\
$\beta_{3}$ & -19.57 & -14.42 & -18.92 & -13.92 \\
$S . e$. & $(5.96)$ & $(5.89)$ & $(5.96)$ & $(5.89)$ \\
$R^{2}$ & 0.97 & 0.97 & 0.91 & 0.91 \\
$n$ & 909 & 3707 & 909 & 3707 \\
\hline Noncitizen/foreign-born/Hispanic & & & \\
$\beta_{3}$ & -18.36 & -14.75 & -17.71 & -14.28 \\
$S . e$. & $(5.92)$ & $(5.93)$ & $(5.92)$ & $(5.93)$ \\
$R^{2}$ & 0.98 & 0.97 & 0.93 & 0.92 \\
$n$ & 913 & 3771 & 913 & 3771 \\
\hline Noncitizen/foreign-born & & & \\
$\beta_{3}$ & -9.93 & -8.22 & -9.33 & -7.77 \\
$s . e$. & $(3.92)$ & $(3.54)$ & $(3.92)$ & $(3.58)$ \\
$R^{2}$ & 0.98 & 0.98 & 0.93 & 0.93 \\
$n$ & 924 & 3874 & 924 & 3874 \\
\hline Control & Region & All & Region & All \\
\hline Nos & & & & \\
\hline
\end{tabular}

Notes: Panel A (B): Each estimate is from a separate OLS regression with the (proportion of the) log of population of the corresponding demographic group for state-month-year combinations as the dependent variable. Population includes all CPS data (using given sampling weights) for individuals ages 20 to 60 . All regressions include state and month-year effects, controlling for any existing systematic differences across states as well as any differences over time that may affect all states' outcomes. Column (1) reports estimates using the regional control groups, while column (2) employs all U.S. states as a control. Huber-White robust standard errors are reported in parentheses, allowing for unrestricted error correlations across observations within states. States having previously adopted omnibus legislation are excluded from the control groups. The estimate reported is the interaction between the indicator for 1 to 6 months post-implementation and the treatment indicator.

Table 1.5. Difference-in-differences estimates of the impact of state omnibus immigration laws on employment: immigrants
A. $100 \times \ln ($ employment level $)$
B. $100 \times \ln ($ employment proportion)

(1)

(2)

(1)

(2)

\begin{tabular}{|c|c|c|c|c|}
\hline \multicolumn{5}{|c|}{-skilled } \\
\hline$\beta_{3}$ & -20.73 & -16.32 & -21.17 & -17.03 \\
\hline s.e. & (7.34) & $(7.08)$ & $(7.22)$ & (7.08) \\
\hline$R^{2}$ & 0.95 & 0.96 & 0.89 & 0.88 \\
\hline$n$ & 899 & 3627 & 899 & 3627 \\
\hline
\end{tabular}

Noncitizen/foreign-born/arrived to U.S. after 1982/Hispanic 


\begin{tabular}{|c|c|c|c|c|}
\hline $\begin{array}{l}\beta_{3} \\
\text { s.e. } \\
R^{2} \\
n\end{array}$ & $\begin{array}{l}-19.52 \\
(7.55) \\
0.96 \\
904\end{array}$ & $\begin{array}{l}-16.42 \\
(7.25) \\
0.96 \\
3715\end{array}$ & $\begin{array}{l}-19.96 \\
(7.44) \\
0.90 \\
904\end{array}$ & $\begin{array}{l}-17.16 \\
(7.20) \\
0.89 \\
3715\end{array}$ \\
\hline \multicolumn{5}{|c|}{ Noncitizen/foreign-born/arrived to U.S. after 1982} \\
\hline $\begin{array}{l}\beta_{3} \\
\text { s.e. } \\
R^{2} \\
n\end{array}$ & $\begin{array}{l}-12.89 \\
(4.98) \\
0.98 \\
923\end{array}$ & $\begin{array}{l}-9.12 \\
(4.50) \\
0.98 \\
3870\end{array}$ & $\begin{array}{l}-13.41 \\
(4.83) \\
0.91 \\
923\end{array}$ & $\begin{array}{l}-9.89 \\
(4.45) \\
0.92 \\
3870\end{array}$ \\
\hline $\begin{array}{l}\text { Noncitiz } \\
\beta_{3} \\
\text { s.e. } \\
R^{2} \\
n\end{array}$ & $\begin{array}{l}\text { orn/Hispa } \\
-16.59 \\
(6.61) \\
0.96 \\
907\end{array}$ & $\begin{array}{l}\text { W-skillec } \\
-13.92 \\
(6.18) \\
0.96 \\
3670\end{array}$ & $\begin{array}{l}-17.03 \\
(6.46) \\
0.90 \\
907\end{array}$ & $\begin{array}{l}-14.63 \\
(6.17) \\
0.89 \\
3670\end{array}$ \\
\hline $\begin{array}{l}\text { Noncitiz } \\
\beta_{3} \\
\text { s.e. } \\
R^{2} \\
n\end{array}$ & $\begin{array}{l}\text { orn/Hispa } \\
-15.23 \\
(6.78) \\
0.97 \\
907\end{array}$ & $\begin{array}{l}-13.29 \\
(6.37) \\
0.97 \\
3731\end{array}$ & $\begin{array}{l}-15.67 \\
(6.65) \\
0.91 \\
907\end{array}$ & $\begin{array}{l}-14.02 \\
(6.31) \\
0.90 \\
3731\end{array}$ \\
\hline $\begin{array}{l}\text { Noncitiz } \\
\beta_{3} \\
\text { s.e. } \\
R^{2} \\
n\end{array}$ & $\begin{array}{l}\text { rn } \\
-10.62 \\
(4.48) \\
0.98 \\
924\end{array}$ & $\begin{array}{l}-7.90 \\
(4.07) \\
0.98 \\
3871\end{array}$ & $\begin{array}{l}-11.14 \\
(4.33) \\
0.92 \\
924\end{array}$ & $\begin{array}{l}-8.67 \\
(4.00) \\
0.93 \\
3871\end{array}$ \\
\hline Control & Region & All & Region & All \\
\hline
\end{tabular}

Notes: Panel A (B): Each estimate is from a separate OLS regression with the (proportion of the) log of employment of the corresponding demographic group for state-month-year combinations as the dependent variable. Employment includes all CPS data (using given sampling weights) for individuals ages 20 to 60 . All regressions include state and month-year effects, controlling for any existing systematic differences across states as well as any differences over time that may affect all states' outcomes. Column (1) reports estimates using the regional control groups, while column (2) employs all U.S. states as a control. HuberWhite robust standard errors are reported in parentheses, allowing for unrestricted error correlations across observations within states. States having previously adopted omnibus legislation are excluded from the control groups. The estimate reported is the interaction between the indicator for 1 to 6 months postimplementation and the treatment indicator.

Table 1.6. Difference-in-differences estimates of the impact of state omnibus immigration laws on population: natives

\begin{tabular}{lcccc}
\hline & A. $100 \times \ln ($ population level) & \multicolumn{2}{c}{ B. $100 \times \ln$ (population proportion) } \\
& $(1)$ & $(2)$ & $(1)$ & $(2)$ \\
\hline Native/Hispanic/low-skilled & & & \\
$\beta_{3}$ & -11.57 & -7.16 & -10.97 & -6.71 \\
s.e. & $(7.28)$ & $(6.92)$ & $(7.32)$ & $(6.97)$ \\
$R^{2}$ & 0.95 & 0.96 & 0.92 & 0.91 \\
$n$ & 886 & 3791 & 886 & 3791 \\
\hline
\end{tabular}

Native/white, non-Hispanic/low-skilled 


\begin{tabular}{|c|c|c|c|c|}
\hline $\begin{array}{l}\beta_{3} \\
\text { s.e. } \\
R^{2} \\
n\end{array}$ & $\begin{array}{l}-0.93 \\
(1.18) \\
0.99 \\
924\end{array}$ & $\begin{array}{l}-0.36 \\
(1.21) \\
0.99 \\
3876\end{array}$ & $\begin{array}{l}-0.32 \\
(1.14) \\
0.98 \\
924\end{array}$ & $\begin{array}{l}0.09 \\
(1.24) \\
0.98 \\
3876\end{array}$ \\
\hline \multicolumn{5}{|c|}{ Native/black, non-Hispanic/low-skilled } \\
\hline $\begin{array}{l}\beta_{3} \\
\text { s.e. } \\
R^{2} \\
n\end{array}$ & $\begin{array}{l}6.51 \\
(5.40) \\
0.98 \\
894\end{array}$ & $\begin{array}{l}5.80 \\
(5.24) \\
0.98 \\
3715\end{array}$ & $\begin{array}{l}7.11 \\
(5.30) \\
0.96 \\
894\end{array}$ & $\begin{array}{l}6.23 \\
(5.11) \\
0.95 \\
3715\end{array}$ \\
\hline $\begin{array}{l}\text { Naturali } \\
\beta_{3} \\
\text { s.e. } \\
R^{2} \\
n\end{array}$ & $\begin{array}{l}\text { reign-bor } \\
-4.70 \\
(8.36) \\
0.93 \\
816\end{array}$ & $\begin{array}{l}\text { panic/lo } \\
-7.68 \\
(8.08) \\
0.93 \\
3483\end{array}$ & $\begin{array}{l}-3.99 \\
(8.27) \\
0.83 \\
816\end{array}$ & $\begin{array}{l}-7.22 \\
(7.96) \\
0.81 \\
3483\end{array}$ \\
\hline $\begin{array}{l}\text { Total } \\
\beta_{3} \\
\text { s.e. } \\
R^{2} \\
n\end{array}$ & $\begin{array}{l}-0.61 \\
(0.38) \\
0.99 \\
924\end{array}$ & $\begin{array}{l}-0.45 \\
(0.46) \\
0.99 \\
3876\end{array}$ & $\begin{array}{l}--- \\
--- \\
--- \\
---\end{array}$ & $\begin{array}{l}--- \\
--- \\
--- \\
---\end{array}$ \\
\hline Control & Region & All & Region & All \\
\hline
\end{tabular}

Notes: Panel A (B): Each estimate is from a separate OLS regression with the (proportion of the) log of population of the corresponding demographic group for state-month-year combinations as the dependent variable. Population includes all CPS data (using given sampling weights) for individuals ages 20 to 60 . All regressions include state and month-year effects, controlling for any existing systematic differences across states as well as any differences over time that may affect all states' outcomes. Column (1) reports estimates using the regional control groups, while column (2) employs all U.S. states as a control. Huber-White robust standard errors are reported in parentheses, allowing for unrestricted error correlations across observations within states. States having previously adopted omnibus legislation are excluded from the control groups. The estimate reported is the interaction between the indicator for 1 to 6 months post-implementation and the treatment indicator.

Table 1.7. Difference-in-differences estimates of the impact of state omnibus immigration laws on employment: natives

\begin{tabular}{lcccc}
\hline & A. $100 \times \ln ($ employment level $)$ & \multicolumn{2}{c}{ B. $100 \times \ln ($ employment proportion) } \\
& $(1)$ & $(2)$ & $(1)$ & $(2)$ \\
\hline Native/Hispanic/low-skilled & & & \\
$\beta_{3}$ & -10.89 & -4.05 & -11.46 & -4.82 \\
s.e. & $(10.28)$ & $(10.45)$ & $(10.30)$ & $(10.54)$ \\
$R^{2}$ & 0.94 & 0.95 & 0.90 & 0.89 \\
$n$ & 872 & 3735 & 872 & 3735 \\
\hline
\end{tabular}

Native/white, non-Hispanic/low-skilled 


\begin{tabular}{|c|c|c|c|c|}
\hline $\begin{array}{l}\beta_{3} \\
\text { s.e. } \\
R^{2} \\
n\end{array}$ & $\begin{array}{l}0.73 \\
(1.44) \\
0.99 \\
924\end{array}$ & $\begin{array}{l}2.10 \\
(1.36) \\
0.99 \\
3876\end{array}$ & $\begin{array}{l}0.21 \\
(1.35) \\
0.97 \\
924\end{array}$ & $\begin{array}{l}1.33 \\
(1.30) \\
0.97 \\
3876\end{array}$ \\
\hline \multicolumn{5}{|c|}{ Native/black, non-Hispanic/low-skilled } \\
\hline $\begin{array}{l}\beta_{3} \\
\text { s.e. } \\
R^{2} \\
n\end{array}$ & $\begin{array}{l}7.79 \\
(6.54) \\
0.97 \\
872\end{array}$ & $\begin{array}{l}10.68 \\
(5.80) \\
0.97 \\
3612\end{array}$ & $\begin{array}{l}7.24 \\
(6.56) \\
0.95 \\
872\end{array}$ & $\begin{array}{l}9.93 \\
(5.74) \\
0.93 \\
3612\end{array}$ \\
\hline $\begin{array}{l}\text { Naturali } \\
\beta_{3} \\
\text { s.e. } \\
R^{2} \\
n\end{array}$ & $\begin{array}{l}\text { reign-bo } \\
-14.60 \\
(9.06) \\
0.92 \\
826\end{array}$ & $\begin{array}{l}\text { panic/lo } \\
-16.48 \\
(7.84) \\
0.92 \\
3397\end{array}$ & $\begin{array}{l}-15.14 \\
(8.93) \\
0.83 \\
826\end{array}$ & $\begin{array}{l}-17.47 \\
(7.79) \\
0.79 \\
3397\end{array}$ \\
\hline $\begin{array}{l}\text { Total } \\
\beta_{3} \\
\text { s.e. } \\
R^{2} \\
n\end{array}$ & $\begin{array}{l}-0.52 \\
(0.58) \\
0.99 \\
924\end{array}$ & $\begin{array}{l}-0.77 \\
(0.58) \\
0.99 \\
3876\end{array}$ & $\begin{array}{l}--- \\
--- \\
--- \\
---\end{array}$ & $\begin{array}{l}--- \\
--- \\
--- \\
---\end{array}$ \\
\hline Control & Region & All & Region & All \\
\hline
\end{tabular}

Notes: Panel A (B): Each estimate is from a separate OLS regression with the (proportion of the) log of employment of the corresponding demographic group for state-month-year combinations as the dependent variable. Employment includes all CPS data (using given sampling weights) for individuals ages 20 to 60 . All regressions include state and month-year effects, controlling for any existing systematic differences across states as well as any differences over time that may affect all states' outcomes. Column (1) reports estimates using the regional control groups, while column (2) employs all U.S. states as a control. HuberWhite robust standard errors are reported in parentheses, allowing for unrestricted error correlations across observations within states. States having previously adopted omnibus legislation are excluded from the control groups. The estimate reported is the interaction between the indicator for 1 to 6 months postimplementation and the treatment indicator.

Table 1.8. Difference-in-differences estimates of the impact of state omnibus immigration laws on population and employment (no Oklahoma): immigrants
A. Population
B. Employment

(1)

(2)

(1)

(2)

Noncitizen/foreign-born/arrived to U.S. after 1982/Hispanic/low-skilled

\begin{tabular}{lllll}
$\beta_{3}$ & -31.05 & -26.25 & -19.37 & -18.01 \\
s.e. & $(6.47)$ & $(6.84)$ & $(6.72)$ & $(7.85)$ \\
$R^{2}$ & 0.97 & 0.96 & 0.96 & 0.96 \\
$n$ & 781 & 3117 & 779 & 3073 \\
\hline
\end{tabular}

Noncitizen/foreign-born/arrived to U.S. after 1982/Hispanic 


\begin{tabular}{lllll}
$\beta_{3}$ & -28.88 & -25.41 & -18.55 & -18.58 \\
$S . e$. & $(6.71)$ & $(7.12)$ & $(7.06)$ & $(8.06)$ \\
$R^{2}$ & 0.98 & 0.97 & 0.97 & 0.96 \\
$n$ & 790 & 3189 & 784 & 3149 \\
\hline Noncitizen/foreign-born/arrived to & U.S. after 1982 & & \\
$\beta_{3}$ & -16.49 & -14.53 & -12.60 & -11.17 \\
$s . e$. & $(4.59)$ & $(4.13)$ & $(4.79)$ & $(4.66)$ \\
$R^{2}$ & 0.98 & 0.98 & 0.98 & 0.98 \\
$n$ & 804 & 3286 & 803 & 3282 \\
\hline Noncitizen/foreign-born/Hispanic/low-skilled & & \\
$\beta_{3}$ & -27.38 & -23.01 & -15.54 & -14.97 \\
$S . e$. & $(5.80)$ & $(5.85)$ & $(6.17)$ & $(6.85)$ \\
$R^{2}$ & 0.97 & 0.97 & 0.97 & 0.96 \\
$n$ & 789 & 3147 & 787 & 3113 \\
\hline Noncitizen/foreign-born/Hispanic & & & \\
$\beta_{3}$ & -25.27 & -22.19 & -14.45 & -14.82 \\
$S . e$. & $(5.95)$ & $(6.14)$ & $(6.41)$ & $(7.08)$ \\
$R^{2}$ & 0.98 & 0.97 & 0.97 & 0.97 \\
$n$ & 793 & 3196 & 787 & 3162 \\
\hline Noncitizen/foreign-born & & & \\
$\beta_{3}$ & -13.24 & -12.30 & -10.42 & -9.97 \\
$s . e$. & $(4.09)$ & $(3.58)$ & $(4.33)$ & $(4.19)$ \\
$R^{2}$ & 0.98 & 0.98 & 0.98 & 0.98 \\
$n$ & 804 & 3286 & 804 & 3283 \\
\hline Control & Region & All & Region & All \\
\hline Note & & & & \\
\hline
\end{tabular}

Notes: Estimates correspond to those found in Tables 1.4 and 1.5, excluding Oklahoma's effective date of November 2007 from the treatment group. In addition, I report only population and employment levels, not proportions. Panel A displays estimates associated with population, while Panel B displays estimates associated with employment.

Table 1.9. Difference-in-differences estimates of the impact of state omnibus immigration laws on population and employment (no Oklahoma): natives

\begin{tabular}{lllll}
\hline & \multicolumn{2}{c}{ A. Population } & \multicolumn{2}{c}{ B. Employment } \\
& $(1)$ & $(2)$ & $(1)$ & $(2)$ \\
\hline \multicolumn{2}{l}{ Native/Hispanic/low-skilled } & & & \\
$\beta_{3}$ & -14.53 & -11.49 & -11.27 & -10.71 \\
S.e. & $(7.88)$ & $(7.47)$ & $(10.17)$ & $(9.89)$ \\
$R^{2}$ & 0.95 & 0.96 & 0.94 & 0.95 \\
$n$ & 774 & 3223 & 761 & 3173 \\
\hline
\end{tabular}

Native/white, non-Hispanic/low-skilled 


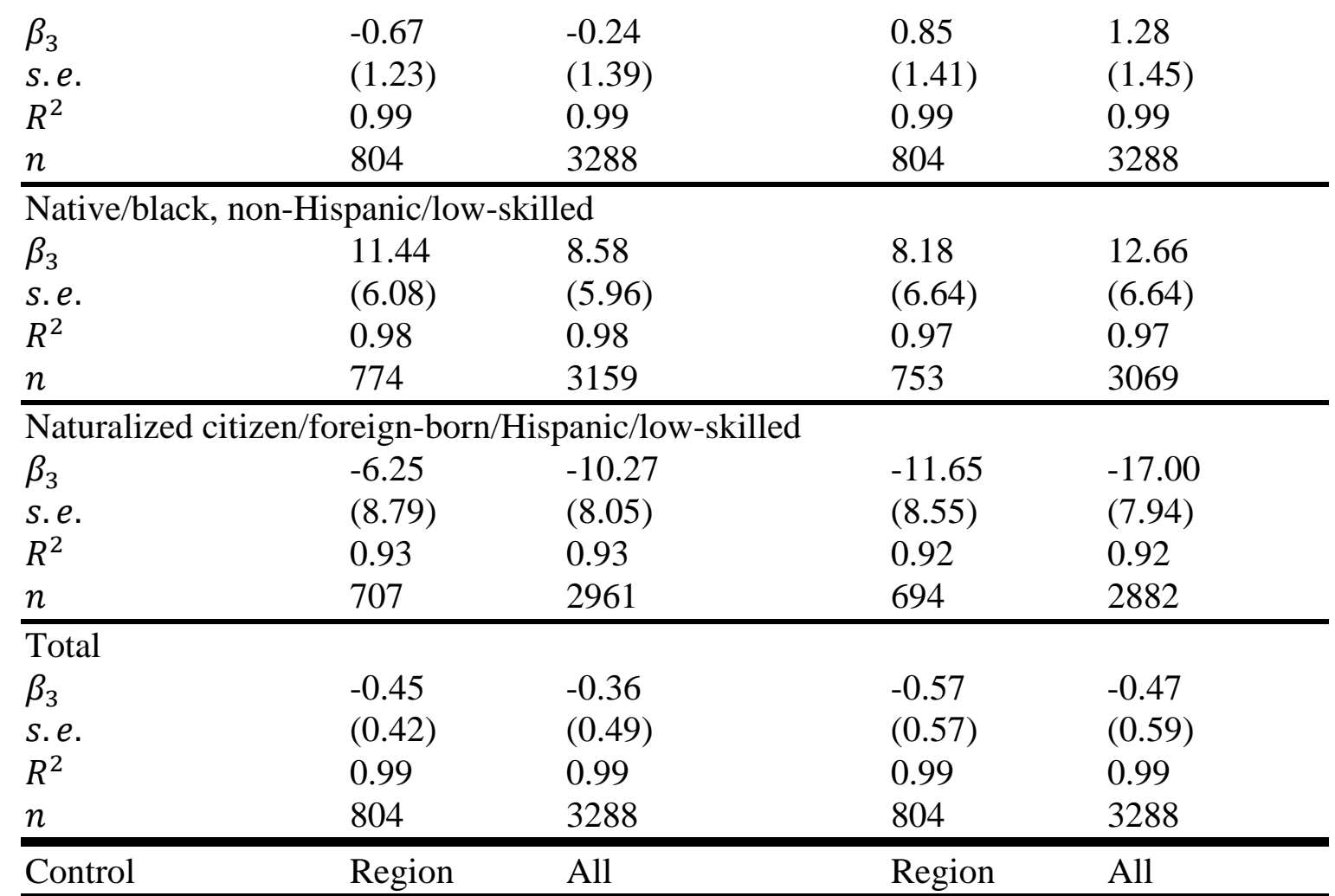

Notes: Estimates correspond to those found in Tables 1.6 and 1.7, excluding Oklahoma's effective date of November 2007 from the treatment group. In addition, I report only population and employment levels, not proportions. Panel A displays estimates associated with population, while Panel B displays estimates associated with employment.

Table 1.10. Difference-in-differences estimates of the impact of state omnibus immigration laws on population and employment: immigrants

A. Six-month implementation period B. Two-year implementation period Population Employment Population Employment
(1)
(2)
(3)
(4)
(1)
(2)
(3)
(4)

\begin{tabular}{|c|c|c|c|c|c|c|}
\hline \multicolumn{7}{|c|}{ Noncitizen/foreign-born/arrived to U.S. after 1982/Hispanic/low-skilled } \\
\hline$\beta_{3}$ & $-24.75-19.29$ & $-10.09-14.60$ & -7.11 & -6.38 & -4.63 & -2.38 \\
\hline s.e. & (9.54) (10.57) & $(8.81)(10.38)$ & (5.08) & (5.23) & (5.95) & $(6.1$ \\
\hline$R^{2}$ & 0.97 & 0.96 & 0.96 & 0.96 & 0.95 & 0.96 \\
\hline
\end{tabular}




\begin{tabular}{|c|c|c|c|c|c|c|c|c|}
\hline$n$ & 454 & 1846 & 457 & 1817 & 1751 & 7216 & 1737 & 7117 \\
\hline \multicolumn{9}{|c|}{ Noncitizen/foreign-born/arrived to U.S. after 1982/Hispanic } \\
\hline$\beta_{3}$ & -21.97 & -16.54 & -12.10 & -16.89 & -8.26 & -8.96 & -6.51 & -5.75 \\
\hline s.e. & $(9.38)$ & $(10.70)$ & $(8.93)$ & $(10.12)$ & $(5.11)$ & $(5.08)$ & $(6.05)$ & (6.13) \\
\hline$R^{2}$ & 0.98 & 0.97 & 0.97 & 0.96 & 0.97 & 0.97 & 0.95 & 0.96 \\
\hline$n$ & 459 & 1883 & 459 & 1854 & 1765 & 7392 & 1747 & 7285 \\
\hline \multicolumn{9}{|c|}{ Noncitizen/foreign-born/arrived to U.S. after 1982} \\
\hline$\beta_{3}$ & -10.90 & -8.71 & -9.55 & -11.24 & -5.07 & -4.17 & -2.50 & -2.41 \\
\hline s.e. & $(6.25)$ & $(5.86)$ & $(6.46)$ & $(5.89)$ & $(3.15)$ & $(3.04)$ & $(3.59)$ & $(3.55)$ \\
\hline$R^{2}$ & 0.98 & 0.98 & 0.98 & 0.98 & 0.98 & 0.98 & 0.98 & 0.98 \\
\hline$n$ & 462 & 1936 & 462 & 1934 & 1801 & 7629 & 1795 & 7617 \\
\hline \multicolumn{9}{|c|}{ Noncitizen/foreign-born/Hispanic/low-skilled } \\
\hline$\beta_{3}$ & -22.28 & -17.71 & -10.85 & -14.74 & -3.60 & -2.84 & -1.10 & -0.86 \\
\hline s.e. & $(8.54)$ & $(9.05)$ & $(8.40)$ & $(9.21)$ & $(4.56)$ & $(4.65)$ & $(5.24)$ & $(5.41)$ \\
\hline$R^{2}$ & 0.97 & 0.97 & 0.97 & 0.96 & 0.96 & 0.97 & 0.95 & 0.96 \\
\hline$n$ & 455 & 1860 & 460 & 1839 & 1760 & 7263 & 1747 & 7178 \\
\hline \multicolumn{9}{|c|}{ Noncitizen/foreign-born/Hispanic } \\
\hline$\beta_{3}$ & -19.32 & -14.56 & -11.59 & -15.53 & -5.27 & -5.77 & -3.43 & -2.62 \\
\hline s.e. & $(8.24)$ & $(9.23)$ & $(8.20)$ & $(9.15)$ & $(4.52)$ & $(4.47)$ & $(5.35)$ & $(5.40)$ \\
\hline$R^{2}$ & 0.98 & 0.97 & 0.97 & 0.97 & 0.97 & 0.97 & 0.96 & 0.96 \\
\hline$n$ & 459 & 1886 & 460 & 1863 & 1768 & 7405 & 1751 & 7314 \\
\hline \multicolumn{9}{|c|}{ Noncitizen/foreign-born } \\
\hline$\beta_{3}$ & -8.60 & -7.51 & -8.10 & -10.36 & -1.98 & -1.34 & 0.42 & 0.31 \\
\hline s.e. & $(5.57)$ & $(5.19)$ & $(5.76)$ & $(5.49)$ & $(2.84)$ & $(2.71)$ & (3.27) & $(3.21)$ \\
\hline$R^{2}$ & 0.98 & 0.98 & 0.98 & 0.98 & 0.98 & 0.98 & 0.98 & 0.98 \\
\hline$n$ & 462 & 1936 & 462 & 1934 & 1801 & 7629 & 1798 & 7623 \\
\hline Control & Region & All & Region & All & Region & n All & Region & All \\
\hline
\end{tabular}

Table 1.11. Difference-in-differences estimates of the impact of state omnibus immigration laws on population and employment: natives

A. Six-month implementation period B. Two-year implementation period Population Employment Population Employment
(1)
(2)
(3) (4)
(1) (2)
(3)
(4)

\begin{tabular}{lllllllll}
\hline \multicolumn{2}{l}{ Native/Hispanic/low-skilled } & & & & & & \\
$\beta_{3}$ & -24.60 & -21.30 & -2.45 & -12.28 & -4.75 & -2.56 & -7.98 & -5.70 \\
S.e. & $(10.57)$ & $(9.87)$ & $(13.58)$ & $(13.32)$ & $(6.20)$ & $(5.91)$ & $(7.12)$ & $(6.73)$ \\
$R^{2}$ & 0.96 & 0.96 & 0.96 & 0.96 & 0.95 & 0.96 & 0.94 & 0.95
\end{tabular}




\begin{tabular}{|c|c|c|c|c|c|c|c|c|}
\hline$n$ & 446 & 1900 & 449 & 1875 & 1729 & 7463 & 1701 & 7354 \\
\hline \multicolumn{9}{|c|}{ Native/white, non-Hispanic/low-skilled } \\
\hline$\beta_{3}$ & -3.23 & -2.89 & -2.72 & -0.30 & -0.59 & -0.43 & -1.55 & -1.40 \\
\hline s.e. & $(1.37)$ & $(1.61)$ & $(2.27)$ & $(2.02)$ & $(0.90)$ & $(0.89)$ & $(1.07)$ & $(1.04)$ \\
\hline$R^{2}$ & 0.99 & 0.99 & 0.99 & 0.99 & 0.99 & 0.99 & 0.99 & 0.99 \\
\hline$n$ & 462 & 1938 & 462 & 1938 & 1801 & 7632 & 1799 & 7632 \\
\hline \multicolumn{9}{|c|}{ Native/black, non-Hispanic/low-skilled } \\
\hline$\beta_{3}$ & 5.10 & 4.16 & 13.17 & 15.59 & 0.47 & -1.75 & 0.33 & -0.05 \\
\hline s.e. & (7.81) & (7.27) & (10.62) & $(8.54)$ & $(4.20)$ & $(4.08)$ & (4.73) & (4.68) \\
\hline$R^{2}$ & 0.98 & 0.98 & 0.97 & 0.97 & 0.98 & 0.98 & 0.97 & 0.97 \\
\hline$n$ & 448 & 1858 & 433 & 1818 & 1743 & 7329 & 1696 & 7104 \\
\hline \multicolumn{9}{|c|}{ Naturalized citizen/foreign-born/Hispanic/low-skilled } \\
\hline$\beta_{3}$ & 6.51 & 6.64 & -1.05 & -3.15 & 8.55 & 4.03 & -1.81 & 5.97 \\
\hline S.e. & $(11.49$ & $(11.63)$ & $(12.22)$ & $(12.40)$ & $(6.20)$ & $(5.83)$ & $(3.05)$ & $(6.38)$ \\
\hline$R^{2}$ & 0.93 & 0.93 & 0.94 & 0.92 & 0.92 & 0.93 & 0.91 & 0.92 \\
\hline$n$ & 411 & 1735 & 417 & 1691 & 1589 & 6818 & 1565 & 6618 \\
\hline \multicolumn{9}{|l|}{$\overline{\text { Total }}$} \\
\hline$\beta_{3}$ & -0.65 & -0.54 & -0.45 & -1.04 & 0.06 & 0.09 & 0.26 & 0.12 \\
\hline s.e. & $(0.43)$ & $(0.58)$ & $(0.62)$ & $(0.50)$ & $(0.28)$ & $(0.34)$ & $(0.43)$ & $(0.44)$ \\
\hline$R^{2}$ & 0.99 & 0.99 & 0.99 & 0.99 & 0.99 & 0.99 & 0.99 & 0.99 \\
\hline$n$ & 462 & 1938 & 462 & 1938 & 1801 & 7632 & 1799 & 7632 \\
\hline Control & Regiol & All & Region & All & Regio & All & Regior & All \\
\hline
\end{tabular}

Notes: Estimates correspond to those found in Tables 1.6 and 1.7, however resulting from either six-month or two-year total implementation periods. Panel A displays estimates associated with the six-month window, while Panel B displays estimates associated with the two-year window. I report only population and employment levels, not proportions.

Table 1.12. Rankings one year before implementation

\begin{tabular}{lllll}
\hline State & \multicolumn{2}{l}{ Population } & \multicolumn{3}{l}{ Employment } \\
\hline Georgia & $3 / 12$ & $9 / 49$ & $3 / 12$ & $8 / 49$ \\
Oklahoma & $7 / 10$ & $27 / 49$ & $8 / 10$ & $28 / 49$ \\
Missouri & $6 / 11$ & $27 / 44$ & $8 / 11$ & $25 / 44$ \\
South Carolina & $9 / 11$ & $26 / 44$ & $8 / 11$ & $25 / 44$ \\
Utah & $3 / 10$ & $4 / 44$ & $3 / 10$ & $4 / 44$ \\
Nebraska & $4 / 11$ & $21 / 44$ & $7 / 11$ & $30 / 44$ \\
Arizona & $3 / 10$ & $4 / 44$ & $3 / 10$ & $5 / 44$
\end{tabular}


Notes: Population (employment) is measured as the proportion of low-skilled, Hispanic immigrants arriving to the US after 1982 among the total population (employed) in each state (ages 20 to 60), with a ranking of one designating the highest proportion.

Table 1.13. Estimates of the impact of the narrowest demographic on state immigration legislation implementation

\begin{tabular}{|c|c|c|c|c|c|}
\hline & $\begin{array}{r}\text { A. Perce } \\
\text { narrowest }\end{array}$ & $\begin{array}{l}\text { of } \\
\text { graphic }\end{array}$ & $\begin{array}{l}\text { B. Change in p } \\
\text { narrowest }\end{array}$ & $\begin{array}{l}\text { entage of } \\
\text { nographi }\end{array}$ & $\begin{array}{l}\text { fic } \\
\text { ic }\end{array}$ \\
\hline & Population & Employment & Population & Employ & yment \\
\hline & $(1)$ & (3) & $(1)$ & (3) & (4) \\
\hline Coefficient & 0.51 & 0.34 & -0.24 & -0.22 & -0.04 \\
\hline $\begin{array}{l}\text { S.e. } \\
\text { 95\% bounds: }\end{array}$ & $(0.40) \quad(0.10)$ & $(0.44) \quad(0.11)$ & $(0.31) \quad(0.08)$ & $(0.34)$ & $(0.08)$ \\
\hline Lower & $-0.27 \quad-0.04$ & $-0.53 \quad-0.12$ & $-0.85-0.19$ & -0.89 & 0.20 \\
\hline Upper & 1.28 & 0.29 & $\begin{array}{ll}0.37 & 0.10 \\
\end{array}$ & 0.45 & 0.12 \\
\hline Control & Region All & Region All & Region All & Region & All \\
\hline $\begin{array}{l}\text { Notes: Each est } \\
\text { legislation imple } \\
\text { for Panel A: a o } \\
\text { (employment) is } \\
\text { legislation imple } \\
\text { increase in the ch } \\
\text { is associated w } \\
\text { implementation. }\end{array}$ & $\begin{array}{l}\text { e is from an } \\
\text { tation as the d } \\
\text { nit increase in } \\
\text { sociated with } \\
\text { tation. Report } \\
\text { e in the percent } \\
\text { a } 100 \% * \text { coef }\end{array}$ & $\begin{array}{l}\text { ression with th } \\
\text { variable. Report } \\
\text { ntage of the na } \\
\text { coefficient incre } \\
\text { tes have the fol } \\
\text { narrowest demo } \\
\text { crease in the }\end{array}$ & $\begin{array}{l}\text { amy variable fo } \\
\text { timates have the } \\
\text { st demographic } \\
n \text { the probabili } \\
\text { g interpretation } \\
\text { hic in total state } \\
\text { bility of state }\end{array}$ & $\begin{array}{l}\text { iibus im } \\
\text { ving inte } \\
\text { al state p } \\
\text { state im } \\
\text { anel B: a } \\
\text { tion (emp }\end{array}$ & $\begin{array}{l}\text { migration } \\
\text { rpretation } \\
\text { population } \\
\text { migration } \\
\text { a one unit } \\
\text { ployment) }\end{array}$ \\
\hline
\end{tabular}

Figure 1.1. Population before and after implementation: narrowest demographic 

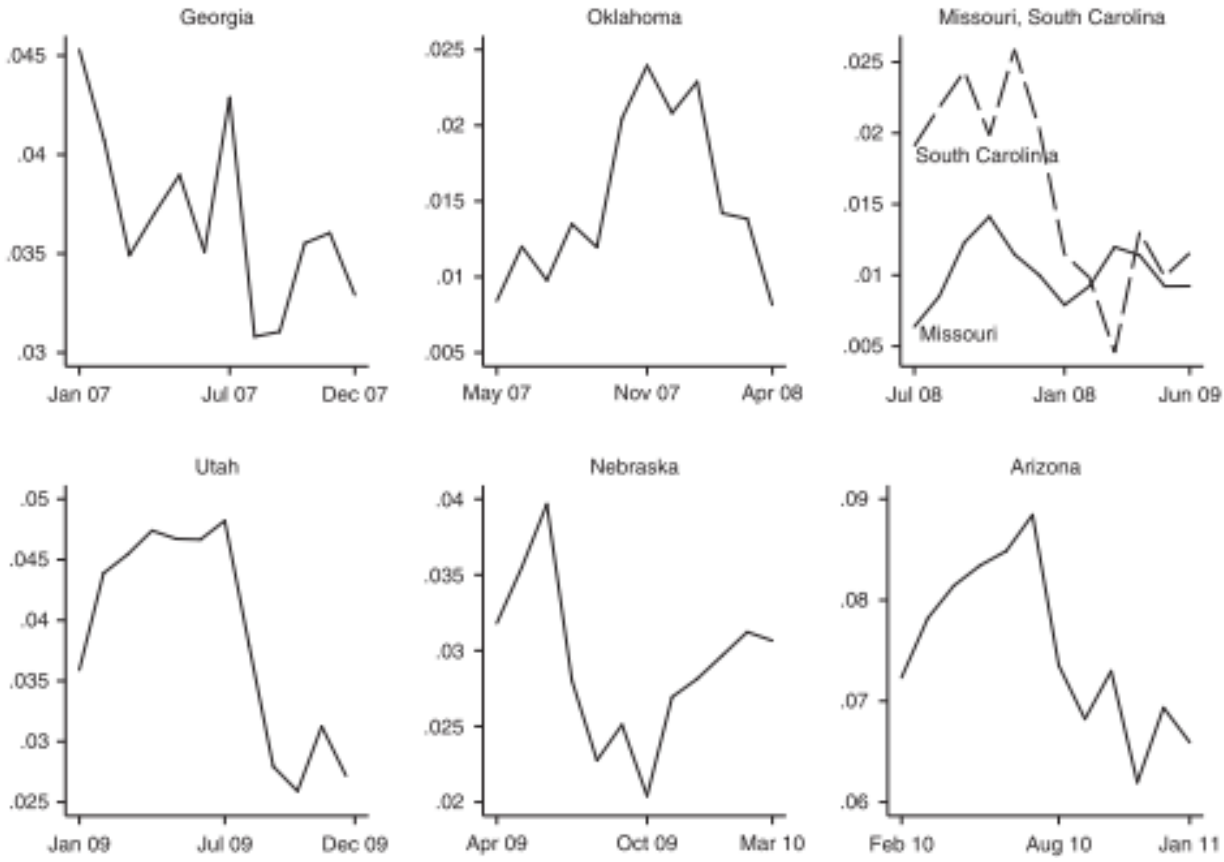

Notes: Population is measured as the proportion of low-skilled, Hispanic immigrants arriving to the US after 1982 in the total state population (ages 20 to 60). Proportions displayed are measured during the one-year implementation period, consisting of six months before and after the implementation date of state omnibus immigration legislation. Implementation month-year combinations are indicated in the middle of the $\mathrm{x}$-axis of each panel.

Figure 1.2. Employment before and after implementation: narrowest demographic 

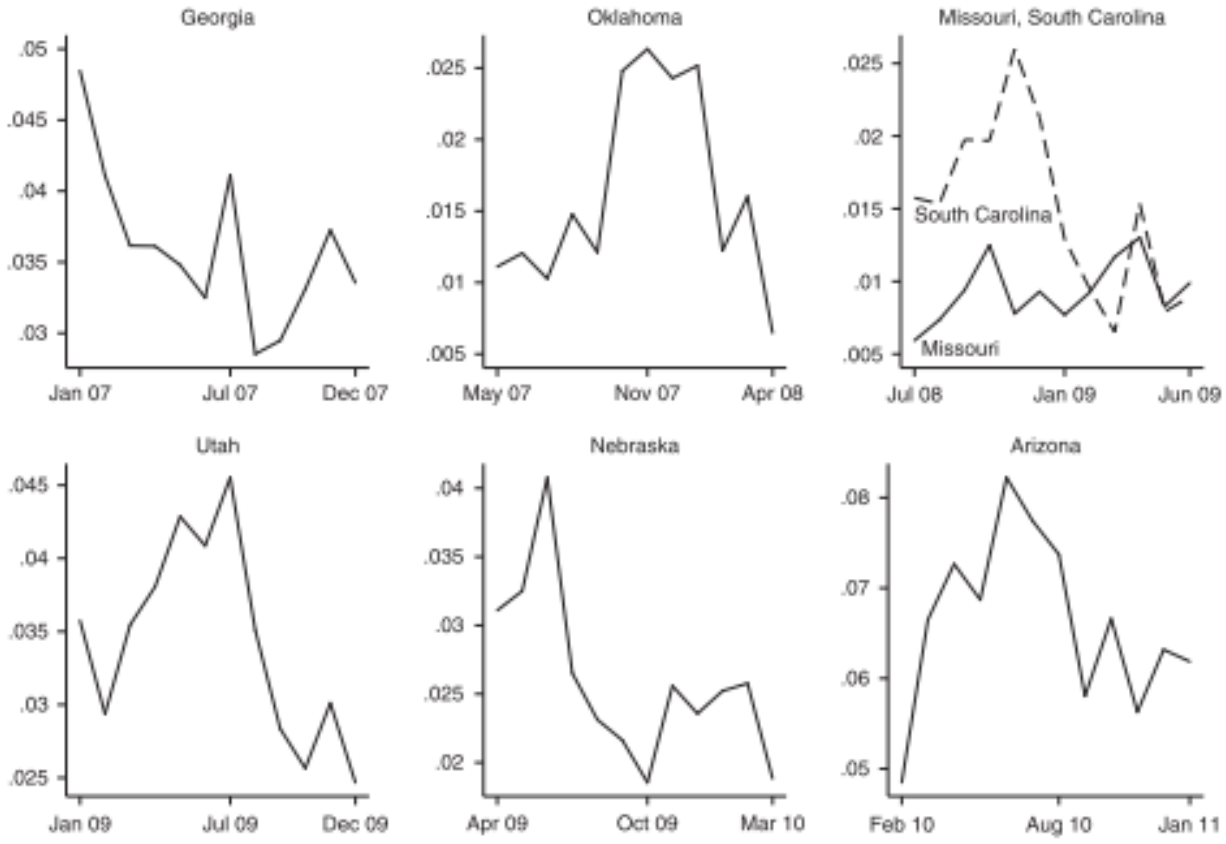

Notes: Employment is measured as the proportion of low-skilled, Hispanic immigrants arriving to the US after 1982 among the total employed in each state (ages 20 to 60). Proportions displayed are measured during the one-year implementation period, consisting of six months before and after the implementation date of state omnibus immigration legislation. Implementation month-year combinations are indicated in the middle of the $\mathrm{x}$-axis of each panel.

Figure 1.3. Population at implementation: narrowest demographic 

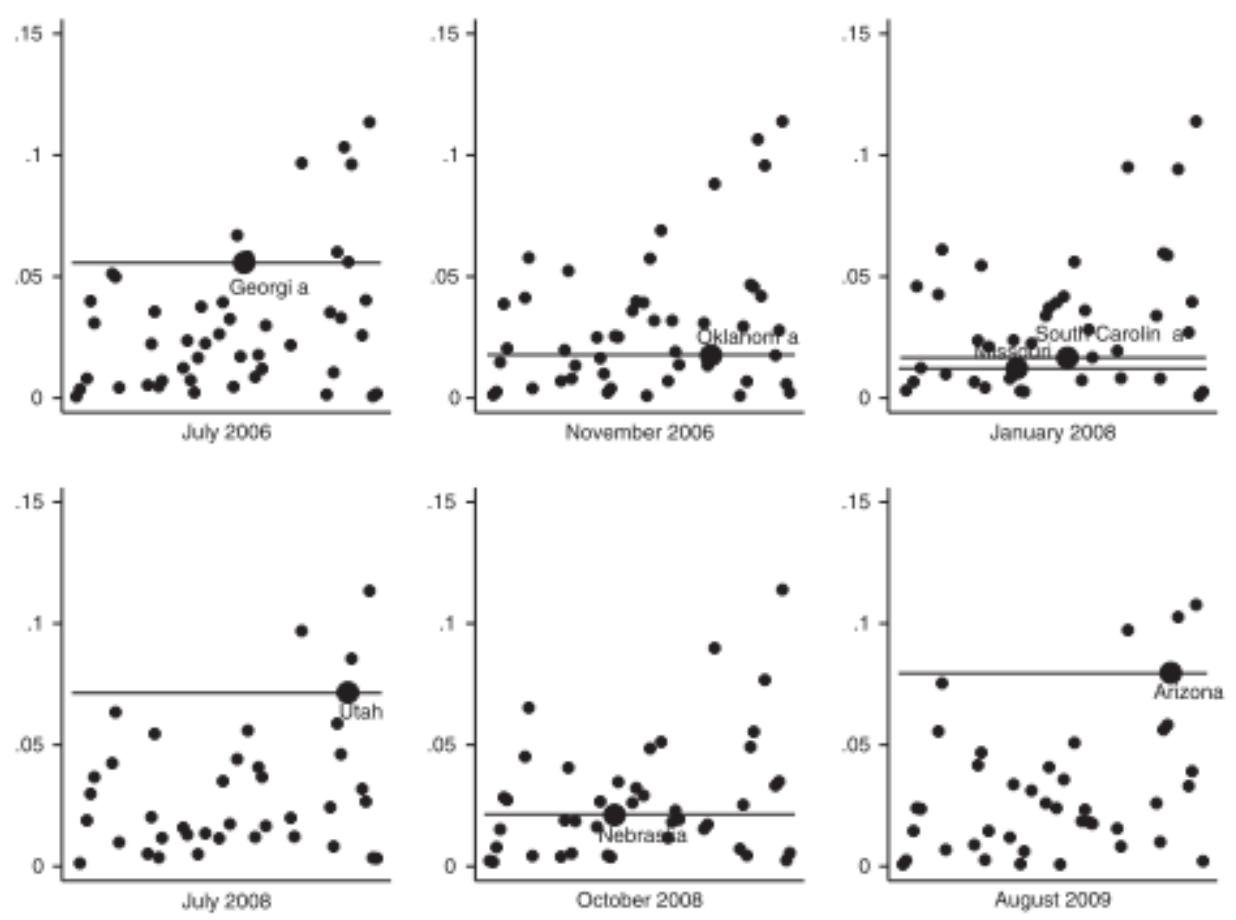

Notes: Population is measured as the proportion of low-skilled, Hispanic immigrants arriving to the US after 1982 in the total state population (ages 20 to 60). Proportions displayed are measured one year before implementation of omnibus immigration law for each state implementing legislation, as well as the corresponding non-implementing states for each given implementation period.

Figure 1.4. Employment at implementation: narrowest demographic 

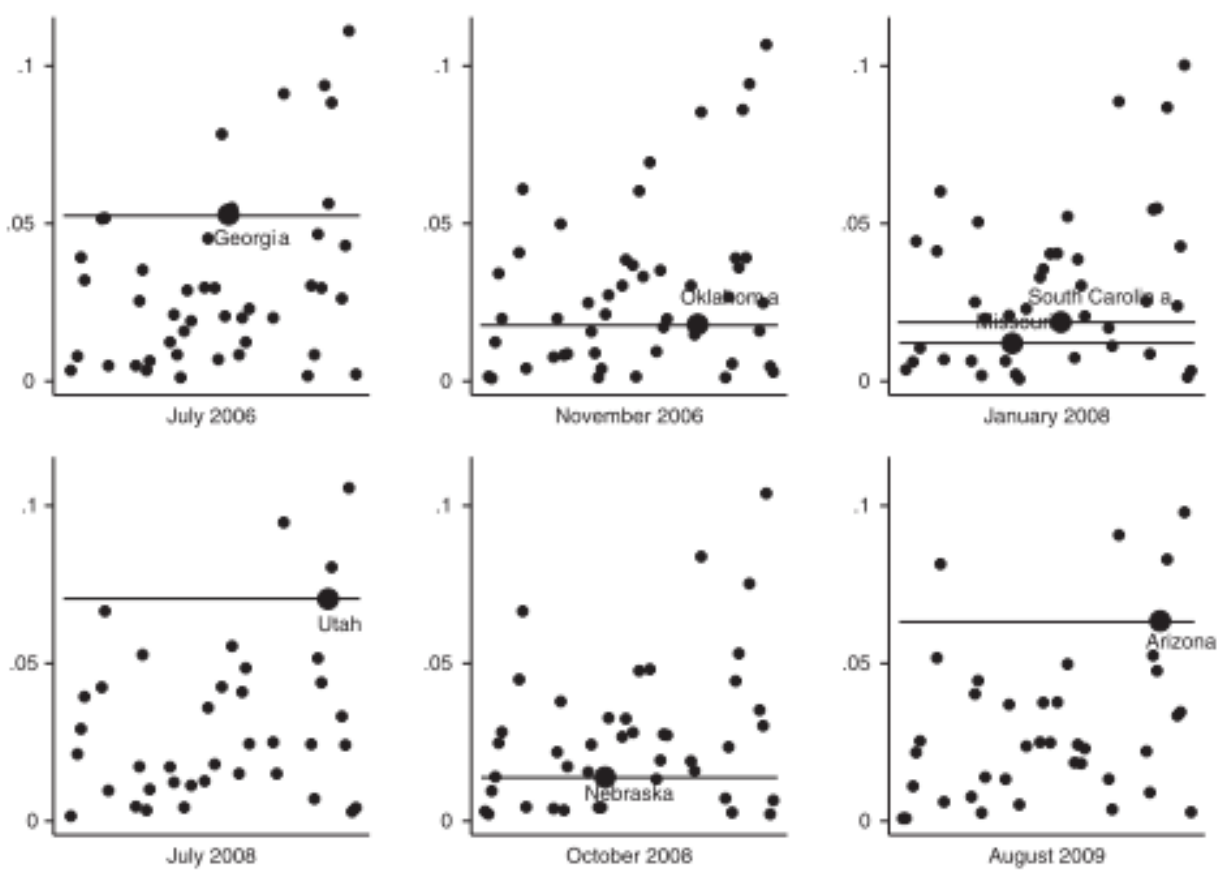

Notes: Employment is measured as the proportion of low-skilled, Hispanic immigrants arriving to the US after 1982 among the total employed in each state (ages 20 to 60). Proportions displayed are measured one year before implementation of omnibus immigration law for each state implementing legislation, as well as the corresponding non-implementing states for each given implementation period.

\section{CHAPTER II}




\section{GEOGRAPHIC PROXIMITY AND THE PRO-TRADE EFFECT OF MIGRATION: STATE-LEVEL EVIDENCE FROM MEXICAN MIGRANTS IN THE UNITED STATES}

\section{Introduction}

Recent studies emphasize the link between migration and trade, both through theoretical models and empirical evidence. From the seminal works of Gould (1994) and Head and Ries (1998) to more recent articles such as Aleksynska and Peri (2011), Kugler and Rapoport (2011) and Hatzigeorgiou and Lodefalk (2011), all point to the same general conclusion: migrants do indeed promote international trade between the destination and origin countries. The robust positive relationship between human and goods mobility across studies is especially noteworthy given the variety of approaches employed and the number of countries studied. While most studies use a standard gravity model augmented with a migration variable as well as various controls for bilateral trade costs, specific methods vary, including pooled cross section or panel data OLS with fixed effects, 2SLS and generalized propensity scores. The US and Canada garner the majority of attention in terms of country-specific studies, focusing on migrants to the destination country and the subsequent trade from the destination country to all other countries; however, studies have also focused on the UK, Spain, Denmark and Bolivia, among others. ${ }^{19}$ Furthermore, the geographic unit under examination varies, many measuring links at the country-country level while others narrow the focus to state-country connections. ${ }^{20}$

\footnotetext{
${ }^{19}$ For example, see Girma and Yu (2002), Peri and Requeña (2010), White (2007), and Erlich and Canavire Bacarreza (2006), respectively.

${ }^{20}$ In order to maintain consistency, I always refer to the geographic unit in terms of destination-origin. For example, I classify a study examining the connection between migrants living in the United States and their
} 
As to the channels through which migrants enhance trade, the consensus points to a preference channel normally associated with increased imports for the migrantdestination country, and an information channel associated with increased imports and exports for the migrant-destination country. Migrants may bring preferences for specific products with them to the destination region, leading to increased imports from the corresponding origin regions; on the other hand, migrants familiar with language, tastes, customs, or the workings of business and law in both the place of origin and destination may pass this information on to firms, thereby lowering the cost associated with entering or increasing presence in a particular foreign market, potentially increasing both imports and exports.

Two natural questions arise from this consistent body of evidence on the pro-trade effect of migration: (1) How localized are the preferences and information that migrants embody and potentially transmit to firms? and (2) How does geographic proximity matter for migration's pro-trade effect? While several previous studies focus on the state level for the given destination country, to my knowledge no studies examine the migration-trade link at the relatively localized state-state level, neither for one migration variable nor considering classification by geographic proximity of migrants.

By employing the state-state level in the following analysis, this finer geographic disaggregation allows for (1) more precise measurement of migrant networks and other potential determinants of trade, including the distance and mass variables fundamental to any gravity model, and (2) differentiation between different states of residence and origin

connection with exports from the U.S. (entire country) to countries of migrants' origins as "countrycountry.” 
for migrants and the associated trade. The former more accurately depicts the variation in the covariates theorized to drive the migration-trade nexus, in turn permitting more precise estimates. ${ }^{21}$ The latter crucially allows for the possibility that networks of migrants of similar state origin matter for the pro-trade effect of migration, above and beyond the traditionally-examined networks of compatriots. In other words, not only does a Mexican migrant now provide different information to the potential U.S. export market than a Canadian migrant, a Veracruzano (from the Gulf coast state of Veracruz, Mexico) also provides different knowledge than a Jalicense (from the Pacific coast state of Jalisco, Mexico).

As far as geographic proximity, Herander and Saavedra (2005) and Artal-Tur et al. (2012) (hereafter HS and APR, respectively) both point to the importance of distance between the networks of migrants that ultimately supply the pro-trade effect of migration as a determinant of the extra associated trade. Further understanding the importance of geographic proximity clearly sheds light on the influence of migrants, specifically as to whether an increase in exports can be expected given an increase in migrants of a particular state in states adjacent to the state under examination. While both previous studies show that geographic proximity certainly matters (the pro-trade effect is stronger as distance between migrant networks decreases), they lack the finer geographic disaggregation on the side of migrant origin (export destination).

My approach examines empirically the migration-trade nexus at the state-state level, first intentionally ignoring geographic proximity and then classifying migrants into

\footnotetext{
${ }^{21}$ For example, Yilmazkuday (2013) finds that the estimated coefficient for distance in the gravity-type models of determinants of international trade suffers from greater bias if not considering production location within countries (at the state or local level).
} 
three categories based on distance between migrant networks. Using data linking migrants' states of origin with current states of residence, I determine how localized the information that migrants transmit to exporting firms actually is and how geographic proximity matters in this process of transmission. Specifically, this method maps Mexican migrants' Mexican state of origin to current state of residence in the United States, using data on matrícula consular (consulate registration) holders available from the Mexican government. ${ }^{22}$ Use of a standard gravity model augmented to include migration as an additional explanatory variable for exports from U.S. to Mexican states, as well as an extension relying on the generalized propensity scores (GPS) method, allow for estimation of the pro-trade effect that migrants have at the state-state level for the first time, both overall and classified by geographic proximity.

I find that just as in previous studies at the country-country and state-country levels, migrants indeed have a statistically significant pro-trade effect at the state-state level, promoting exports from U.S. states of residence to Mexican states of origin. The initial specification reveals an elasticity of exports to migration of 0.11 , while the GPS extension signals a diminishing yet positive marginal contribution over nearly the entire range of actual measured migrant stocks. Classified by geographic proximity, the preferred specification reveals the expected partial pro-trade effects otherwise disguised by the single estimate of migration's pro-trade effect, corresponding to contributions of \$1984 (in-state) and \$538 (neighboring-state) to annual exports between respective U.S. and Mexican states

\footnotetext{
${ }^{22}$ The matrícula consular is an identification card made available by the Mexican government to citizens residing abroad starting in 1871 . The card must be renewed every five years, giving the holder access to opening a bank account, obtaining a driver's license, and other services, depending on the specific country and state of residence. I define state of origin as the last state of permanent residence before migration occurred from Mexico to the U.S..
} 
associated with each average additional migrant. Given these results, geographic proximity appears to matter just as in HS and APR, with the in-state effect surpassing the neighboringstate effect in magnitude; however, in contrast with previous results, I find that the neighboring-state contribution of migrants is indeed of statistical significance. All results vary minimally in magnitude and significance across several checks for robustness, providing clear evidence as to the additional benefit of migration that manifests itself through its nexus with international trade, highlighted at the relatively localized state-state level for the first time and classified by geographic proximity of migrant networks.

The rest of this chapter continues with a discussion of several aspects unique to the U.S.-Mexico relationship with relevance to the migration-trade nexus. I highlight my initial empirical strategy and data sources, followed by a discussion of the main results. After various checks for robustness and the details on the GPS extension, I briefly conclude.

\section{US-Mexico relationship}

The U.S.-Mexico relationship provides an especially interesting and appropriate setting conducive to examining the pro-trade effect of migration for several reasons. First and foremost is the fact that data is actually available, permitting the analysis at the statestate level for the first time. Detailed exports data from U.S. to Mexican states are available for all years since 1994, coinciding with the implementation of the North American Free Trade Agreement. Perhaps most noteworthy is the availability of the matrícula consular data, uniquely allowing for the connection of Mexican state of origin to U.S. state of residence for each migrant registered during the period examined.

Additionally, as both the U.S. and Mexico are relatively large, heterogeneous countries, separation by state origin and destination is theoretically worthwhile; there is 
clearly wide differentiation within countries as to preferences and the knowledge and information that residents hold about markets, customs, and tastes, all important factors for the theorized channels through which the pro-trade effect operates. For example, an emigrant leaving the southeastern state of Chiapas to reside in a given U.S. state undoubtedly has much different information than an emigrant leaving the northern state of Sonora, arguably similar to the level of differentiation existing across migrants of varying nationalities signaled by the previous literature.

Although this differentiation, depending on Mexican state of origin and U.S. state of residence, points unequivocally to the theorized pro-trade effect of migration at the statestate level, several aspects of the U.S.-Mexico relationship signal that this effect could potentially be minimized relative to the entire range of possible pro-trade effects across all countries (and the respective sub-national divisions). First, both trade and migration between the U.S. and Mexico are relatively well-established, neither phenomenon being particularly new in its existence. HS, among others, find that the existence of a previous large migrant stock reduces any pro-trade effect of new migrants. However, the "newness” of migration from and to particular Mexican and U.S. states, respectively, could potentially offset the fact that the Mexico-U.S. migration is not novel at the country-country level. ${ }^{23}$ Second, Mexican migration levels to the U.S. are relatively high, especially relevant if beyond a certain level of migration, further migrants may not marginally stimulate trade between places of residence and origin. The mean state-state count of matrículas

${ }^{23}$ Card and Lewis (2007) examines the choice of U.S. states of destination for Mexican migrants, analyzing changing trends during the 1990s. Hatzigeorgiou and Lodefalk (2011) also highlights the importance of new migrants in updating information. 
consulares for my sample is 3038 , with the maximum of 270,201 corresponding to those Michoacanos registered in California. Previous evidence is divided on the existence of an "exhaustion point" of the pro-trade effect of migration, one of the possibilities explored in the GPS extension of Section 6; Serrano and Requeña (2013, hereafter SR) finds that every migrant makes a positive marginal contribution to exports at the province-country level, while Egger et al. (2012, hereafter EVN) finds evidence of an exhaustion point for imports at the country-country level of approximately 4,000 migrants, above which additional migrants provide zero stimulus for imports. Finally, a majority of the Mexican migrant population in the U.S. is relatively low-skilled and may not participate in any form of business network. These general characteristics are of potential importance given recent findings that being high-skilled and having access to business networks makes migrants particularly effective in their promotion of trade. ${ }^{24}$

Given the outlined aspects of the U.S.-Mexico relationship, any pro-trade effect found at the state-state level between states of these neighbor countries can be hypothesized to fall at the lower end of the spectrum of potential worldwide effects across all countries. In turn, while any claims of external validity should clearly be met with due skepticism, the presence of a U.S.-Mexico link between migration and trade would appear to suggest an even stronger potential of the general existence of a pro-trade effect of migration at the state-state level.

\section{Empirical Strategy and Data}

\footnotetext{
${ }^{24}$ See Felbermayr and Jung (2009) and Aleksynska and Peri (2011) for these respective emphases.
} 
In estimating the effect of migration on international trade between states in the United States and in Mexico, I first employ a standard gravity model, the most common empirical strategy for studies examining not only factors affecting trade, but also the potential pro-trade stimulus provided by migration. Putting aside geographic proximity for a moment, I start with the standard model augmented with migration between respective state pairs as an additional explanatory variable; the resulting specification serves as a useful benchmark for the analysis to follow.

$$
\ln T_{j}^{i}=\alpha+u_{i}+m_{j}+\phi \ln M i g_{j}^{i}+\beta_{1} \ln Y_{i} Y_{j}+\beta_{2} \ln D i s t_{j}^{i}+\beta_{3} A d j_{j}^{i}+e_{j}^{i}
$$

Incorporating the potential importance of geographic proximity, I then further augment the gravity model to include two additional measures of Mexican migration to the United States.

$$
\begin{aligned}
\ln T_{j}^{i}=\alpha+v_{i} & +\mu_{j}+\gamma_{1} \ln M i g_{j}^{i}+\gamma_{2} \ln M i g_{j}^{a d j}+\gamma_{3} \ln M i g_{j}^{r e s t}+\delta_{1} \ln Y_{i} Y_{j}+\delta_{2} \ln D i s t_{j}^{i} \\
& +\delta_{3} A d j_{j}^{i}+\varepsilon_{j}^{i}
\end{aligned}
$$

The variable $T_{j}^{i}$ measures exports from U.S. state $i$ to Mexican state $j$ in terms of yearly total value, dependent on migration, size of market (income), distance, and adjacency. $M i g_{j}^{i}$ captures the stock of matrícula consular holders in each U.S. state $i$ from each Mexican state of origin $j, M i g_{j}^{a d j}$ measures the stock of matrícula consular holders in the states adjacent to each U.S. state $i$ from each Mexican state of origin $j$, while $M i g_{j}^{r e s t}$ reflects the stock of matrícula consular holders in the rest of U.S. states (exclusive of state $i$ and adjacent states) from each Mexican state of origin $j$; $Y_{i}$ and $Y_{j}$ are the gross state products of U.S. state $i$ and Mexican state $j$, respectively, Dist $t_{j}^{i}$ represents the distance by land from U.S. state $i$ capital to Mexican state $j$ capital, while $A d j_{j}^{i}$ is a dummy variable 
taking the value of 1 for adjacent states and that of 0 for states not sharing a border. Given the $\log$ transformations, the coefficients of interest, $\gamma_{1}, \gamma_{2}$, and $\gamma_{3}$ thus pinpoint the percentage increase (decrease) in yearly exports flowing from a U.S. state to a Mexican state associated with a $1 \%$ increase in the stock of migrants originating from the corresponding Mexican state and registered in the corresponding U.S. state (i.e., in-state), in the neighbors of the corresponding U.S. state (i.e., neighboring-state), and in the remainder of all other U.S. states (i.e., other-state), respectively. Additionally, $u_{i}, m_{j}, v_{i}$, and $\mu_{j}$ are U.S. and Mexican state fixed effects, controlling for the multilateral resistance terms as recommended by Anderson and Van Wincoop (2003). Other variables commonly employed as controls for bilateral trade costs in previous migration-augmented gravity models, such as trade agreements, language, colonial ties, legal system, currency, and cultural distance, are not relevant in the current setting since these variables are generally not differentiated within a single country, this being true in the case of the United States and Mexico. ${ }^{25}$ If migration indeed is conducive to trade, an expectation of $\phi>0, \gamma_{1}>0$, $\gamma_{2}>0$, and $\gamma_{3}>0$ holds, the latter two inequalities depending on how far-reaching migrant networks' influence is; if geographic proximity matters for the migration-trade nexus, the clear expectation is $\gamma_{1}>\gamma_{2}>\gamma_{3}$.

Before detailing the data, the use of migrant stocks as the measure of migration merits a brief discussion. While much of the literature relies on migrant flows and subsequent panel data, I follow Felbermayr and Toubal (2007) and Vézina (2012) in the

\footnotetext{
${ }^{25}$ There is a limited amount of heterogeneity for these potential variables, for example with the presence of a number of languages in Mexico, however in the sample at hand this differentiation is so minimal that it does not justify inclusion in the regression equation as an additional control variable.
} 
use of stocks and the resulting cross-sectional data; several reasons exist pointing to migrant stocks as the appropriate choice for measuring migration when examining the migration-trade nexus. ${ }^{26}$ First, the hypothesized information channel operates with an indeterminate amount of lag; it is apparent that some time is needed for migrants to transmit information to exporting firms, however the exact amount of time is unclear, leaving the pinpointing of the appropriate lag for a panel estimation next to impossible. Second, any potential yearly data noise is minimized by employing migration stocks rather than flows. Finally, one may argue that migrant flows capture the most relevant information for the migration-trade link, as each year's migrants hold the most updated information corresponding to the associated year's exports. However, given that my migrant stock is the accumulation of those migrants registering for the matrícula consular between 2006 and 2010, any worries of the embodied information being outdated are easily dispelled, as it is difficult to imagine this type of information rendered useless in the passage of a maximum of only five years. In fact, stocks appropriately capture the notion that exporting firms likely build on existing information accumulated from migrants over the years (even if the information is updated gradually) and that trade relationships may persist even if there is yearly variation in migration flows (Vézina, 2012).

Values of state-state exports are obtained from the U.S. Bureau of Transportation statistics; these statistics cover all exports from the U.S. to Mexico at the state-state level, except for those transported by air or water, providing $90 \%$ coverage of total exports

\footnotetext{
${ }^{26}$ I discuss below how the use of cross-sectional data limits direct treatment of the questions of potential endogeneity and direction of causality in the migration-trade context.
} 
between the two nations. ${ }^{27}$ Given this coverage, the non-contiguous U.S. states of Alaska and Hawaii are excluded from the analysis. ${ }^{28}$ For the preferred specification, trade is measured by the state-state values from the year 2010. As original export data are listed with current dollars as the unit, I use the U.S. CPI-U series to convert all values to 2011 U.S. dollars. Statistics on the number of matrículas consulares issued are calculated given the information provided by the Insitituto de los Mexicanos en el Exterior (IME). Since holders of the card must specify last state of Mexican residence as well as current state of U.S. residence during the application process, these statistics uniquely allow for the construction of the necessary state-state migration data. As the identification cards have a renewal period of five years, I sum the available data from 2006 to 2010 in constructing the stock of Mexican migrants for each state-state combination. ${ }^{29}$ I consult the U.S. Bureau of Economic Analysis (BEA) and the Mexican Instituto Nacional de Estadística y Geografía (INEGI) statistics for the respective gross state products corresponding to 2010, while distance between capital cities is calculated using the shortest route by land expressed in number of miles. ${ }^{30}$ The original data for Mexican gross state products are listed with the unit of 2003 pesos, therefore I initially convert the values to 2003 U.S. dollars using the

\footnotetext{
${ }^{27}$ While imports data would clearly provide for useful comparison, unfortunately state-state data is not presently available.

${ }^{28}$ Modifications to the trade value calculation used to check for sensitivity of the results to these changes are detailed below.

${ }^{29}$ As of final revisions, data from 2010 is the most recent available.

${ }^{30}$ This differs from the standard measure used by similar studies, that of great circle distance, due to the fact that the trade data (and a majority of Mexico-U.S. migration) is by land. However, if great circle distance is indeed employed as the measure of distance, results change only minimally, with a slight increase in the magnitude of the distance coefficient.
} 
average of monthly historical peso-dollar exchange rates from 2003. Finally, just as with the U.S. gross state products originally reported with the unit of 2005 U.S. dollars, I again use the CPI-U series to convert all values to 2011 dollars in order to maintain uniformity with the export values.

As the matrícula consular data do not completely cover the population of Mexican origin in the U.S. and could possibly present problems of selection, I closely examine the distribution of Mexican migrants across the U.S. states of residence in attempting to determine whether these data sufficiently represent the actual distribution of residents of Mexican origin across the U.S. states. As there is no justifiable reason to expect that Mexican state of origin affects selection into obtaining a matrícula consular, ${ }^{31}$ if the distribution of the data is sufficiently close to the actual distribution of Mexican migrants (irrespective of Mexican state origin) across U.S. states, the use of the matrícula consular data can be said to provide a certain level of representativeness for the state-state distribution, thereby minimizing any bias arising from selection problems. I thus can use the 2010 U.S. Census as a benchmark for comparison; I contrast the matrícula consular data with that of the Census, in which the number of residents in each U.S. state claiming Mexican origin is detailed.

Figure 2.1 details the distribution of Mexican migrants in the U.S. for both the matrícula consular data and the Census data; the data are expressed as the number of Mexican migrants in each state divided by the total stock of migrants from each respective

\footnotetext{
${ }^{31}$ A natural assumption may be that education level is associated with legal migration status, thereby making it more likely for individuals to obtain a matrícula consular if the state of origin corresponds to a low-education Mexican state on average. However, this assumption does not appear to be correct; see below for a related brief discussion.
} 
source. ${ }^{32}$ In fact, the matrícula consular data performs well in representing the actual distribution of Mexican migrants across U.S. states, with most states' difference coordinates close to zero. Only two states, Texas and Illinois, suffer from differences greater than 3\%, while 43 of 48 states' differences in percentages are less than $1 \%$.

Additionally, one may expect that the number of highly-educated migrants is underrepresented in the matrícula consular data, because of the fact that there is no clear incentive for a documented U.S. resident to hold the identification card. The consideration is especially important given the previously mentioned studies emphasizing the extra relevance of highly-educated migrants in promoting trade above and beyond the average migrant contribution. Taking the average education level in Mexican states from INEGI statistics, dispersed over a range of 6.7 to 10.5 years of schooling with a mean of 8.6, a first check of the data indeed shows a negative correlation between Mexican state average education level and the percentage of origin state population registered with the matrícula consular. ${ }^{33}$

However, the correlation gives no information as to the key question of how education level actually relates to legal migration status, and in turn to the matrícula consular. It is not clear that the expectation of underrepresentation is reasonable, given that the correlation between legal migration status and education level is anything but definitive for Mexican migrants in the U.S. Passel and Cohn (2009) determines that $47 \%$ of unauthorized migrants ages 25 to 64 in the U.S. have completed high school or less, while

\footnotetext{
${ }^{32}$ This fraction with an upper limit of 1 is then multiplied by 100 , resulting in the numbers expressed on the y-axis of Figure 1.

${ }^{33}$ See Figure 2.2 for a scatter plot of this correlated data.
} 
Caponi and Plesca (2012) argues that documented Mexican migrants in the U.S. are actually more likely to have a lower education level than undocumented migrants. Comparing the matrícula consular data with other representative data as to education level presents two problems. The IME only reports state-state statistics including education level for 2006 and 2007, thereby providing a smaller sample in representing the overall stock of migrants; in addition, the best data for comparison, that of the U.S. Current Population Survey, is known to undercount undocumented migrants. Because of these difficulties and lack of available data, I do not empirically address the issue of state-state distribution by education level. Additional data availability would clearly allow for future exploration of this further rich level of detail.

Table A.1 details the number of matrículas consulares registered from 2006 to 2010, classified by both US state of residence and Mexican state of origin, while Table A.2 focuses on the state-state makeup of Mexican migration to the three top U.S. destination states, California, Texas, and Illinois, and the corresponding exports to Mexico. Table A.2 and Figure A.1 provide an initial idea of the simple correlation between state-state migration and exports. Without any controls for bilateral trade costs or state fixed effects, the best-fit line displayed in Figure A.1 exhibits a slope of 0.44, providing preliminary evidence of a potential positive relationship between migration and exports at the statestate level. Table 2.1 shows the mean, standard deviation, maximum and minimum for variables in both the base and alternative samples.

\section{Results and Discussion}


Column 1 of Table 2.2 displays the results of the OLS regression employing the benchmark gravity equation. Including Mexico City, the base sample consists of 1536 observations, a result of all trading pairs of 48 U.S. and 32 Mexican states. The coefficient estimate of migrants' effect on state-state exports is indeed significantly positive; holding all other factors constant, an increase of $1 \%$ in the number of state-state migrants is associated with a $0.11 \%$ increase in state-state exports, with $p<0.01$. Distance, as expected, is significantly negative, reflecting a $1.60 \%$ decrease in state-state exports associated with a $1 \%$ increase in distance between the respective capitals of U.S. and Mexican states. Holding other factors constant, states that are adjacent enjoy more than double the trade of nonadjacent states, while a $1 \%$ increase in combined economy size is associated with a $0.94 \%$ increase in state-state exports. All coefficient estimates have the expected positive (negative) relationship with state-state exports, and are highly significant.

While these initial results are the first to confirm the existence of the pro-trade effect of migration at the more localized state-state level, given the crucial findings of HS and APR and the main hypothesis of this paper, it is not surprising that Columns 2 to 5 unmask key complexities disguised by the estimates in Column 1. Focusing on the preferred specification in Column 5, the geographic proximity of migrants clearly appears to matter for the promotion of international trade. The coefficients of both in-state and neighboringstate migration are statistically significant, remaining so even after adding all relevant control variables. As expected, the elasticity with respect to in-state migration at 0.07 is lower than that of Column 1, due to the addition of neighboring-state migration. An increase of $1 \%$ in neighboring-state migration is associated with a $0.08 \%$ increase in state- 
state exports, while increased migration in the rest of U.S. states is associated with a small, yet statistically insignificant negative effect on state-state exports. The remaining independent variables' coefficients and levels of significance are similar to those of Column 1, with attenuation in magnitude only for the distance estimate. Interpreting the coefficients of interest, Column 1 appears to capture the overall pro-trade effect of migration, masking the actual importance of geographic proximity. Column 5 sheds light on this importance; in-state migration indeed promotes trade between U.S. states of residence and Mexican states of origin, however neighboring-state migration also has an essential role in this expansion of trade.

The OLS estimates in turn permit a simple calculation of the magnitude of the protrade effect of migration, highlighting the quantitative importance of this effect, as well as allowing for a comparison of the relative sizes of the benefit from in-state, neighboringstate, and other-state migration. This exercise carries extra importance given the fact that at first glance the estimates in Column 5 appear to point to a counterintuitive result, that in-state migration is associated with a smaller pro-trade effect than that of neighboringstate migration. Starting with results from Column 1, given a $10 \%$ increase in average immigration from a particular Mexican state to a particular U.S. state, the average migrant stock increases from 3038 to 3342. Employing the estimated coefficient of approximately 0.11 , this $10 \%$ increase in migration results in an increase in average state-state exports, settling on the new value of exports equal to $\$ 82,923,424$. This translates into $\$ 3061$ extra state-state exports per year associated with the average extra migrant. ${ }^{34}$

\footnotetext{
${ }^{34}$ This figure can be alternatively calculated by multiplying the elasticity of exports to migration by the ratio of average state-state exports to average migrant stock.
} 
When different geographic proximities are separated in Column 5, I can now break down the distinct components of the pro-trade effect of migration. Once again assuming a $10 \%$ increase in average in-state migration, the average in-state migrant stock increases 3038 to 3342 . Using the estimated coefficient of approximately 0.07 results in an increase in average state-state exports of $\$ 602,653$, translating into $\$ 1984$ extra state-state exports per year associated with the average extra in-state migrant. At the same time, these extra 304 migrants are neighboring-state migrants for an average of 4.39 states (the average number of adjacent states for all U.S. states). Given the average neighboring-state migrant stock of 12,409 , this increase is equivalent to a $2.45 \%$ increase. In turn, relying on the percentage increase and the estimate from Column 5 of approximately 0.08 , average statestate exports increase by $\$ 538$ per neighboring-state migrant. Finally, the extra 304 migrants are equivalent to a $0.23 \%$ increase in migration in the rest of the average of 42.61 states. Given the Column 5 estimate of approximately -0.02 , this increase results in a decrease of $\$ 13$ per average migrant. ${ }^{35}$ Finally, collecting all calculations of separate components in order to compile an overall effect results in \$3792 of extra state-state exports associated with the average extra immigrant from Mexico residing in the U.S.; it is crucial to emphasize that this contribution to exports by the single average extra migrant is actually spread across U.S. states, the three terms of Footnote 18 corresponding to state $i$, neighbors of state $i$, and the rest of U.S. states, respectively. ${ }^{36}$ The compiled result is similar in magnitude to that of Column 1, however the separation of distinct geographic

\footnotetext{
${ }^{35}$ Trade diversion could be a simple explanation for this negative effect, however I intentionally do not explore this further given the small magnitude and lack of significance of the $M i g_{j}^{\text {rest }}$ estimate.

${ }^{36}$ The calculation is as follows: $\$ 1984+\$ 538(4.39)-\$ 13(42.61)$.
} 
proximities allows for the differentiation between the relatively larger in-state contribution of the average extra immigrant, the smaller neighboring-state contribution, and the minuscule other-state reduction of state-state exports.

In comparing the estimates and magnitude of the pro-trade effect of migration to those of the literature, I rely on HS and APR, as well as previous state-country and countrycountry studies, as this paper is the first to examine the state-state level. While doing so provides a framework within which viewing my results is feasible, given the novel statestate level and the emphasis on distinct geographic proximities, any comparison must be accompanied by a disclaimer highlighting these differences in sampling and geographic disaggregation of data. Table 2.3 provides an update of Table 2.1 from Peri and Requeña (2010) in order to include estimates from more recent studies, and those of this paper, as well as a comparison of extra annual exports generated per extra migrant. The elasticity of exports to in-state migration estimated as 0.07 echoes the positive, significant estimates from both HS and APR, however my significantly positive estimate for neighboring-state migration contrasts with that of HS (positive, but insignificant) and APR (not significantly different from zero).

Additionally, the finding of $\$ 3792$ extra yearly exports generated by each extra migrant is similar to those of $\$ 2608$ and $\$ 2717$, detailed in White (2007) and Felbermayr and Jung (2009), respectively. While these estimates differ most dramatically from that of $\$ 24,895$ found by Aleksynska and Peri (2011), it is worthwhile to signal that these numbers are not necessarily incompatible. As Aleksynska and Peri (2011) points out, factors such as average number of migrants in the sample and the specific measure of migrant stock contribute to these differentiated estimates. My measure based on the matrícula consular 
includes some migrants who may not be economically active, and does not classify migrants based on education level, which most likely further attenuates estimates as mentioned in Kugler and Rapoport (2011).

\section{Robustness and Sensitivity Checks}

Among concerns of econometrics, it would be ideal to establish the direction of causality given the potential issue of endogeneity. While migrants contribute to trade through the aforementioned channels, there is also the possibility that trade could encourage migration, especially if increased trade is associated with higher incomes that act to attract migrants. However, as pointed out in Felbermayr and Toubal (2012), income and therefore cross-country (state) wage differentials may be affected by aggregate trade, but it is not clear how bilateral trade affects these wage differentials. While I am unable to test for the direction of causality given the cross-sectional data that I employ, I rely on the established literature that has gone to great lengths in demonstrating that rather than trade encouraging migration, migration actually encourages trade. Furthermore, given that my data is at the state-state level, a central concern as to validity expressed in Hanson (2010) is dispelled. Specifically, the state-state data eliminates the concern of omitted variables related to bilateral economic policies driving both international trade and migration since these very economic policies are generally set at the national level, not exhibiting variation within countries.

As a first check for robustness of the obtained results, I outline a new set of estimates in Table 2.4, now excluding Mexico City from the sample given the fact that it is not clear whether Mexico City should be included as a 32nd Mexican "state;" the revised sample contains 1488 state-state observations. The magnitude and significance of the pro- 
trade effect of migration change minimally, the exclusion of Mexico City slightly decreasing the magnitude of the in-state coefficient to just under 0.07 and that of neighboring states to nearly 0.08 . An additional concern arises from the comparison of the matrícula consular data and the U.S. Census data highlighted in Section 3. Although a high level of representativeness is present, Texas and Illinois clearly are outliers in this respect, reflecting a difference of $9.11 \%$ and $3.24 \%$ between the data sets, respectively. Especially given the fact that both Texas and Illinois are two of the main destination states in the U.S. for Mexican migrants, it is important to consider migration's pro-trade effect excluding the two outliers from the sample as an additional test of robustness. Table 2.4 also highlights the coefficient estimates generated excluding Texas and Illinois, using a sample of 1472 observations resulting from the combination of 46 U.S. and 32 Mexican states. Compared to the results presented in Table 2.2, in-state migration's effect on state-state exports is slightly greater, while neighboring-state migration's estimate is slightly lower, both minimally less significant.

Selection of state-state exports from the year 2010 as the measure for the dependent variable could be driving the obtained results; if estimates of migration's pro-trade effect on trade differ greatly across the use of varied individual years of trade data as alternative dependent variables, this would clearly be cause for concern. However, the estimates in fact vary only minimally when using exports data from 2008 and 2009 in lieu of 2010, as reported in Table 2.5. Migration's pro-trade effect remains significant and similar in magnitude across all alternative regressions accounted for, with the in-state estimate bottoming out at 0.0731 and peaking at 0.0935 . Using the same simple method of calculation as in the previous section, these figures correspond to an extra \$1819 and \$2053 
of annual exports, respectively, associated with the average extra in-state migrant, not considering the neighboring- and other-state contributions.

An additional concern is that trade and migration could be determined jointly, leaving forwarding the measure of exports as a clear strategy to alleviate this potential problem. ${ }^{37}$ I regress exports for periods $t+1, t+2$, and $t+3$, respectively, with the preferred sample and specification, using all possible corresponding measures of migrant stock (matrícula consular stock) to eliminate any possibility of joint determination. This strategy results in six further regressions; Table 2.6 reports estimation results along with the corresponding exports and migration measures employed in each additional regression. The estimation of migration's pro-trade effect is consistent across these varied measures, both in magnitude and significance, with the elasticity of state-state exports to in-state migration ranging from 0.0789 to 0.1013 .

Finally, I check the consistency of the estimates reported in Column 1 of Table 2.2 given an alternative measure of migration. Specifically, I now use the share of all Mexican migrants residing in U.S. state $i$ who originate from Mexican state $j$ as the proxy for migration rather than migrant stocks. As outlined in Rauch and Trindade (2002) and echoed in Parsons and Vézina (2013), in the context of U.S.-Mexico state-state trade, a higher level of Mexican migrants from state $j$ in U.S. state $i$ increases the number of potential connections between exporting firms from U.S. state $i$ and state $j$ Mexican consumers while a higher migrant share increases the probability of a U.S. state $i$ exporting firm sending products to the associated Mexican state $j$. Table 7 highlights the estimated

\footnotetext{
${ }^{37}$ As Aleksynska and Peri (2011) mentions, since the migration measure is a stock accumulated over years, it is probable that it is determined before trade flows; however I forward exports to assure that joint determination is not a factor.
} 
coefficients given the use of the alternative measure, clearly confirming the significant positive association of migration with trade across the varied samples.

\section{Extension: An Application of Generalized Propensity Scores}

The evidence from EVN and SR points to the existence of nonlinearities in the migration-trade relationship, EVN finding an exhaustion point beyond which further migration no longer makes a positive marginal contribution to international trade. In the extension of the initial OLS examination, I apply generalized propensity scores to a continuous treatment (migrant stock levels), flexibly permitting the existence of nonlinearities. The GPS estimation provides the advantage of describing the pro-trade effect in detail over the entire spectrum of observed migrant stocks as the resulting estimated dose-response function reflects the expected outcome (exports) associated with each and every observed treatment (migrant stocks) under examination, not just the general elasticity of exports to migration. The use of this methodology is particularly attractive given the importance of addressing three central questions of interest in the migration-trade link: (1) is there a minimum level of migration required to generate positive returns (measured in terms of marginal exports), (2) is there a level of migration corresponding to a saturation point, beyond which positive marginal exports are completely exhausted, and (3) is there a certain migrant stock size that maximizes the pro-trade effect of migration.

\section{Method}

As the GPS uses the comparison of those observations demonstrating a certain level of homogeneity across observable characteristics, the method permits correction of the potential bias caused by selection into varying levels of treatment intensity, also allowing

for the estimation of the trade outcomes associated with each of these different levels of 
treatment intensity. Propensity score methods have been applied to binary treatments (Heckman et al., 1997), multiple treatments (Imbens, 2000), and most recently, continuous treatments (Hirano and Imbens, 2004). As I consider differing levels of state-state migrant stocks as widely varying doses across the spectrum of a continuous treatment in a quasiexperimental setting, I rely on the methodology outlined in the latter of the propensity score applications.

Observing treatment doses $T_{i}$, the vector of observable covariates $X_{i}$, and the outcome variable $Y_{i}=Y_{i}\left(T_{i}\right)$ associated with the received treatment for all state-state pairs $i=1, \ldots, N$, the goal of GPS estimation is ultimately pinpointing the dose-response function, $\mu(\tau)=E\left[Y_{i}(\tau)\right]$, interpreted as the average outcome associated with the specific treatment intensity $\tau$. This clearly highlights one advantage of GPS estimation; it allows for the estimation of the average outcome associated with each and every observed treatment intensity of the independent variable of focus.

The central assumption from Hirano and Imbens (2004) is that of weak unconfoundedness for continuous treatments, defined as $Y(\tau) \perp T \mid X$ for all $\tau \in T$, i.e., for all possible realizations of treatment intensity, the outcome variable must reflect conditional independence. Therefore, any difference in treatment intensities is independent of the corresponding outcomes, after controlling for the observable covariates $X$. Assuming $g(\tau, x)$ to be the conditional density of the treatment given the set of covariates, i.e., $g(\tau, x)=f_{T \mid X}(\tau \mid x)$, the GPS is in turn defined as $G=g(T, X)$.

Just as in other applications of the propensity score, the GPS is characterized by a balancing property in which the probability that $T=\tau$ within strata of the GPS is independent of the set of covariates $X$. In turn, removing potential bias requires two steps: 
first, the estimation of the conditional expectation of the outcome given the treatment and the GPS, $\beta(\tau, g)=E[Y \mid T=\tau, G=g]$; second, the estimation of the dose-response function as the average of the conditional expectation over the GPS at a particular treatment intensity, ${ }^{38} \mu(\tau)=E[\beta(\tau, g(\tau, X))]$.

\section{Estimation of the effect of migration on exports}

After logarithmic transformation, the treatment variable of state-state migrant stocks is approximately normal, with skewness of -0.14 and kurtosis of 2.52 , so I assume a normal distribution in estimating the conditional distribution of migration given the vector of chosen covariates: $\ln T_{i} \mid X_{i} \sim N\left(\beta_{0}+X_{i} \beta_{1}, \sigma^{2}\right) . \beta_{1}$ is a column vector, while $X_{i}$ is a row vector consisting of a variety of observable push and pull determinants of treatment intensity. Population and gross state product (GSP) enter $X_{i}$ as logarithmic transformations, as squares of those logarithmic transformations, and as growth variables for both Mexican states of origin and U.S. states of destination, intentionally allowing for a flexible, nonlinear relationship between these measures of market size and migrant stocks. ${ }^{39}$ Furthermore, I include the standard geographic variables of adjacency and distance as covariates; the former as a binary variable taking the value of 1 for adjacent state-state pairs and 0 for non-adjacent state-state pairs, and the latter as a logarithmic transformation of the distance in miles by land from the respective Mexican state capital to the respective U.S.

\footnotetext{
${ }^{38}$ See Hirano and Imbens (2004) for the proof that these two steps actually remove bias.

${ }^{39}$ In other specifications, I also included cubic terms of both population and GSP for Mexican and U.S. states, however, while explanatory power $\left(R^{2}\right)$ increased slightly, none of the additional coefficients exhibited high statistical significance. Estimated coefficients did not change in any significant way compared to those reported.
} 
state capital. ${ }^{40}$ In addition, I insert unemployment rates and Gini coefficients for both Mexican states of origin and U.S. states of destination into the $X_{i}$ vector, controlling for scarcity of employment availability and income inequality, respectively. ${ }^{41}$ Both measures are frequently included as push and pull factors in the determinants of migration (Clark et al., 2007; Grogger and Hanson, 2011; Mayda, 2010). I estimate the equation using scaled ordinary least squares, highlighting the results in Table $2.8 .^{42}$

In general, the selected covariates are highly statistically significant, all independent variables being significant at the $2 \%$ level or lower except for the first- and second-order "Mex. GSP” variables and “Adjacency;” furthermore, the majority of the variation in treatment intensity (size of migrant stocks) is indeed explained by the covariates making up the vector $X_{i}$, the $R^{2}$ signaling this portion as $76 \%$.

With the OLS estimation in hand, I construct the GPS as:

$$
\widehat{G}_{l}=\frac{1}{\sqrt{2 \pi \widehat{\sigma}^{2}}} \exp \left(\frac{1}{2 \widehat{\sigma}^{2}}\left(T_{i}-\widehat{\beta_{0}}-X_{i} \widehat{\beta_{1}}\right)^{2}\right)
$$

After generating the propensity scores, the balancing property must be tested in order to verify that the GPS indeed improves the balance of covariates, thereby providing confirmation of the first step necessary for bias removal. I follow the group and block method suggested by Hirano and Imbens (2004) in carrying out the balance check.

\footnotetext{
${ }^{40}$ This differs from the standard circle distance used by much of the gravity literature, however I choose this measure given that the trade data captures only trade by land and a majority of Mexico-U.S. migration is by land, as well.

${ }^{41}$ I take U.S. population data, U.S. unemployment rates, and U.S. Gini coefficients from the BEA, Census Bureau and Bureau of Labor Statistics, respectively; data for Mexican states come from the INEGI.

${ }^{42}$ Both exports and migration are expressed as logarithms of the respective variable plus one. Results are equivalent to those obtained by maximum likelihood estimation, the method suggested for this initial estimation in Bia and Mattie (2008).
} 
First, dividing the observations into four treatment intensity groups allows for the comparison of covariates across these quartiles of the migrant stock distribution before balancing on the GPS. The four groups of treatment intensity contain 373, 371, 372 and 372 state-state observations, respectively. ${ }^{43}$ As no GPS adjustment has yet occurred, the left section of Table 2.10 clearly reflects the great disparities across groups in the covariates of vector $X_{i}$, showing an average t-statistic of 5.46 and $66 \%$ of observable covariate comparisons across treatment intensity groups being statistically different at the $5 \%$ level. If left alone, these disparities lead to obvious concerns of biased inference due to selection into treatment intensity groups determined by observable characteristics.

Second, dividing the observations into blocks or strata according to the GPS allows for comparison of covariates across the treatment intensity quartiles, but now balanced on the GPS. I evaluate the GPS for all observations $i=1, \ldots, N$ using the OLS estimates at the median level of treatment intensity $T_{m j}$ for each of the four quartiles $j \in\{1,2,3,4\}$, then dividing the propensity scores into ten blocks based on the resulting GPS estimate deciles for each of the four corresponding $T_{m j}$. Just as before adjustment, I carry out two-tailed ttests in order to measure the balance of covariates present comparing across groups, weighting the t-statistics by the respective number of observations in each block. However, having blocked on the GPS estimates allows me to now compare observations that have similar observable characteristics and hence the same predicted treatment intensity (same GPS block), but also have differing levels of actual treatment intensity (different migrant

\footnotetext{
${ }^{43}$ The Appendix details robustness checks using the GPS method with two alternative samples.
} 
stock quartiles). ${ }^{44}$ The middle section of Table 2.11 highlights the vast improvement in balancing the covariates achieved by employing the GPS; 79\% of all covariate comparisons across treatment intensity groups exhibit no statistical differences at the $5 \%$ level, with average t-statistics displayed of 1.22 .

Some studies employing propensity score estimation additionally rely on the common support condition in order to improve comparability of observations. The use of the common support simply results in the exclusion of any observations in the given sample that do not demonstrate a certain level of similarity in the observable covariates. Given the group and block method, this translates into comparing the GPS calculations for $\widehat{G}_{k}\left(T_{m j}, X_{k}\right)$ with those of $\widehat{G}_{l}\left(T_{m j}, X_{l}\right)$, where $k \in j$ and $l \in j$. In turn, the only observations used in the remainder of the estimation process are those $l$ observations where:

$$
\min \left\{\widehat{G}_{k}\left(T_{m j}, X_{k}\right)\right\} \leq \widehat{G}_{l}\left(T_{m j}, X_{l}\right) \leq \max \left\{\widehat{G}_{k}\left(T_{m j}, X_{k}\right)\right\} \text { for all } j \in\{1,2,3,4\} \text {. }
$$

A potential dilemma arises as only 390 of the 1488 state-state observations in fact meet the common support condition stated above. Faced with this large loss of information, a decision must be made among three standard solutions: (1) estimate the outcome variable only within the common support, thereby maximizing the reduction in bias, but also reducing the range over which exports can be predicted given the observed migration levels; (2) estimate the outcome variable inside and outside the common support, thereby maximizing the reduction in bias, maximizing the range over which exports can be predicted given the observed migration levels, but also reducing the preciseness of the estimated outcomes; or (3) estimate the outcome variable with all available observations,

\footnotetext{
${ }^{44}$ The group and block method is highlighted in Tables 2.9 and 2.10, for the entire sample and for the modified common support, respectively.
} 
thereby accepting a non-maximized reduction in potential bias, maximizing the range over which exports can be predicted given the observed migration levels, and maximizing the preciseness of the estimated outcomes. As exhibited in Table 11, much of the potential bias is indeed reduced simply by balancing on the GPS, without any consideration for the common support; $79 \%$ of all covariate comparisons across treatment intensity groups exhibit no statistical differences at the $5 \%$ level. On the other hand, using only the common support observations would greatly reduce the range of observed migrant stocks from 0227,032 (all observations) to 2-5,878 (common support observations), corresponding to a dramatic reduction in means and medians of migrant stocks from 2406 to 347 and 210.5 to 134, respectively. Further evidence as to why the common support condition may be too stringent for our purposes is provided by the simple comparison of covariate means between those state-state combinations inside and outside the common support region. Table 2.12 highlights these comparisons, and perhaps surprisingly, the two groups do not appear to differ dramatically in the means of the covariates.

This fact points to the observation that exclusion from the common support region is mostly attributed to a lack of similarity of generalized propensity scores corresponding to just one of the four median treatment GPS group calculations, not a general lack of comparability of observables for the excluded observations across all four median treatment GPS groups. As the common support equation above highlights, the condition for inclusion in the common support region indeed depends on overlap of the GPS in each and every group $j$, therefore if any state-state observation has even only one exception to this rule, it is automatically excluded by the common support condition. Given the combination of the evidence mentioned, there is no clear best option of the three standard 
solutions, although accepting the trade-off of a non-maximized reduction in bias in exchange for a maximized amount of information, range over which exports can be predicted, and preciseness in estimation may be the most attractive.

However, following Lechner (2008) in exploring alternative solutions to the common support problem, I continue the estimation process by pursuing a fourth option, one that I argue permits researchers confronted with similar common support dilemmas a certain amount of flexibility that is extremely useful in modifying the common support condition according to the particular needs of the research undertaken. By slightly relaxing the stringent condition requiring presence of observations in the common support region for all four groups $j$, we can still assure a maximized reduction of bias, while not trading off as much coverage and preciseness in terms of estimated outcomes. If indeed the standard common support condition means the researcher must sacrifice a large portion of information as in this paper, this condition relaxation provides a second-best option that can be applied to the data. My modified common support rule simply relaxes equation the equation above, now proposing that the only observations used in the remainder of the estimation process are those $l$ observations where:

$$
\min \left\{\widehat{G}_{k}\left(T_{m j}, X_{k}\right)\right\} \leq \widehat{G}_{l}\left(T_{m j}, X_{l}\right) \leq \max \left\{\widehat{G}_{k}\left(T_{m j}, X_{k}\right)\right\} \text { for at least two } j \in\{1,2,3,4\} \text {. }
$$

That is, state-state observations are included as long as the corresponding GPS median-treatment scores for at least two of the four treatment quartiles fall within the common support region of the particular quartile. Guaranteeing a high level of comparability, while relaxing the condition from the original common support equation results in a new sample that does not suffer from the great loss of information previously seen; only 59 state-state observations are excluded by the modified common support, 
resulting in the preferred sample of 1429 observations, and the range of migrant stocks over which exports can be estimated is not reduced at all. ${ }^{45}$ Additionally, the balancing of covariates is greatly improved through the use of the modified common support condition. The right section of Table 2.11 highlights this dramatic improvement; 71 of 72 covariate comparison groups show no statistical differences at the $5 \%$ level, with an average tstatistic of 0.71 . In turn, the high level of comparability of observations vindicates the use of the modified common support for this data set, as the guarantee of comparability of observations and the resulting reduction in bias is the very reason for adhering to a common support condition in the first place.

Moving on with only those observations meeting the modified common support condition, as a first step in estimating the dose-response function, I estimate the conditional expectation of exports given treatment intensity and the corresponding GPS:

$$
E\left[Y_{i} \mid T_{i}, G_{i}\right]=\alpha_{0}+\alpha_{1} T_{i}+\alpha_{2} T_{i}^{2}+\alpha_{3} T_{i}^{3}+\alpha_{4} \widehat{G}_{i}+\alpha_{5} \widehat{G}_{i} T_{i} .
$$

$T_{i}$ values are the actual observed migrant stocks, while $\hat{G}_{i}$ values are those estimates calculated from above. The resulting OLS estimates in Table 2.13 have no direct economic interpretation (Hirano and Imbens, 2004), however the individual and joint statistical

\footnotetext{
${ }^{45}$ The Appendix lists the 59 state-state observations excluded from the $n=1488$ sample, as well as those observations excluded from the $n=1536$ and $n=1426$ samples. It is important to point out that the selection of at least two $j \mathrm{~s}$ and four treatment quartiles is the result of trying several variations of the modified common support condition; however, rather than being a drawback of the approach, this actually provides the researcher with the advantage of flexibility while assuring comparability of observations. In other words, it is easy to consider modified conditions that differ along the two mentioned dimensions, the number of $j s$ and the number of original treatment groups, for example conditions requiring three of four quartiles, four of five quintiles, etc. Much as the Akaike Information Criterion (AIC) provides a means for model selection through trade-off between goodness of fit and model complexity, selection of the appropriate modified common support condition is dictated by the desired trade-off of the amount of balancing and bias reduction sacrificed compared to the amount of estimation power gained under each modification.
} 
significance of the GPS coefficients is noteworthy. ${ }^{46}$ This significance signals that selection is important, confirming that the inclusion of the GPS terms and the GPS estimation process in general are indeed worthwhile in achieving some level of selection bias removal.

Finally, I estimate the dose-response function, capturing the average potential outcome at each and every treatment intensity $\tau$ :

$$
\widehat{E\left[Y_{\tau}\right]}=\frac{1}{N} \sum_{i=1}^{N}\left[\widehat{\alpha_{0}}+\widehat{\alpha_{1}} \tau+\widehat{\alpha_{2}} \tau^{2}+\widehat{\alpha_{3}} \tau^{3}+\widehat{\alpha_{4}} \widehat{G}\left(\tau, X_{i}\right)+\widehat{\alpha_{5}} \hat{G}\left(\tau, X_{i}\right) \tau\right]
$$

I report both the dose-response function and its derivative, the treatment effect function, in Figure 2.3.

\section{Marginal Contributions}

By calculating the marginal exports associated with each treatment level, $\operatorname{MarExp} p=\left(E_{p+1}-E_{p}\right) /\left(M_{p+1}-M_{p}\right)$, where $E$ and $M$ represent estimated exports and actual migrant stocks (backed out from the respective logarithmic transformations) and $p$ denotes the ordinal value of treatment intensities employed in the estimation of the doseresponse function, the nonlinearities present in the exports-migration relationship are now clearly on visual display in Figures 2.4 and 2.5.

Considering the benchmark OLS state-state estimate of \$3061 extra exports per extra migrant, this average contribution of migration to exports is clearly weighted by the first migrants from one respective Mexican state to the state of U.S. residence. The first migrant makes a marginal contribution of $\$ 76,297$ to exports, the hundredth contributes $\$ 3613$, and the thousandth contributes $\$ 175$. Marginal contributions of $\$ 8371$, $\$ 1879$, and

\footnotetext{
${ }^{46}$ I choose to include the GPS only linearly as higher-order GPS terms (squared and cubed) do not add extra information and are not statistically significant.
} 
$\$ 166$, respectively, correspond to the 25th, 50th, and 75th percentiles $(M=33,216$, and 1026) of the actual state-state migrant stock distribution for the $n=1429$ sample. Marginal exports of $\$ 3061$ matches to a migrant stock of 123 , representing the 42nd percentile of the actual state-state migrant stock distribution. Interestingly, at the level of 2276 migrants, the marginal contribution is temporarily “exhausted," and dips below zero until 3761 migrants; this negative marginal contribution to exports bottoms out at $-\$ 4.53$. However, only 66 of the 1429 (less than 5\%) state-state migrant stocks fall in this exhaustion zone range. Total exports quickly recovers from the short-lived negative effect of migration, reaching the pre-exhaustion zone level of exports as early as 4900 migrants, with subsequent migrants all making increasingly positive marginal contributions to statestate exports.

The estimated dose-response and treatment-effect functions provide clear answers to the three central questions of interest posed at the beginning of this paper. First, there is no minimum level of migration (other than one) necessary to generate positive returns in terms of exports; in fact, an individual migrant has the largest pro-trade effect when there are few migrants of the corresponding state-state classification. Second, while there does exist a small range of migrant stocks over which the marginal contribution of migrants turns slightly negative, this negative contribution is extremely temporary, as further migration returns the marginal contribution increasingly positive over the remaining range of state-state migrant stocks. Finally, because the marginal contribution remains positive beyond the exhaustion zone, the pro-trade effect of migration can only be maximized by the maximum level of observed state-state migration.

\section{Conclusion}


Migrants indeed create a significant force in promoting extra trade from U.S. states of residence to Mexican states of origin. This finding is empirically consistent not only in statistical significance, but also in magnitude across the varied methods, specifications, and samples employed in this paper. Without consideration of potential nonlinearities and differing geographic proximities in an augmented gravity model, the elasticity of state-state exports to migration is 0.11 , translating into $\$ 3061$ extra annual exports per average extra migrant for a particular US-Mexico state-state combination, holding other factors constant. On the other hand, the application of generalized propensity scores permits the potential of nonlinearities in the migration-trade relationship, results pointing to a diminishing yet positive marginal contribution of migration to exports as migrant stock size increases over most of the range of measured migrant stocks. These results contribute the first evidence of the pro-trade effect of migration at the state-state level, a relatively localized level capable of measuring more accurately the potential determinants of trade and differentiating between migrant networks of varied state origin.

Additionally, this paper unmasks the importance of distinct geographic proximities that the use of one migration variable disguises. Through the examination of not only instate migration, but also neighboring-state and other-state migration, geographic proximity is revealed to indeed be a relevant factor in determining the pro-trade effect of migration, with networks suffering lower amounts of spatial separation making larger contributions to trade. Both in-state and neighboring-state migration make significantly positive contributions to state-state exports, with estimated elasticities of 0.07 and 0.08 , respectively, resulting in partial contributions to average state-state exports of \$1984 and \$538. Combining the three contributions from migration of separate geographic 
proximities gives an overall addition to state-state exports of $\$ 3792$ by the average extra migrant.

Empirical studies employing data sets from countries other than the U.S. and Mexico, or classifying data by characteristics such as migrants' education level and participation in business networks, offer clear avenues for further research at the state-state level, just as they already have at the country-country level. In addition, sorting trade and migration data by industry, as well as decomposing the pro-trade effect into the intensive and extensive contributions, provides promising extensions. Furthermore, the very existence of the exhaustion zone gives another direction for related research specifically focusing on the GPS method: first, in verifying that a similar shape of the dose-response and treatment-effect functions obtains using other data, and second, in hypothesizing why it is that the exhaustion zone may exist yet does not extend to the larger migrant stocks providing positive contributions to state-state exports. Finally, the results not only shed light on how localized migration's nexus with trade may be and how geographic proximity matters, they inevitably connect to the ongoing debate in a host of countries as to the economic costs and benefits of migration. Without a doubt, the pro-trade effect of both instate and neighboring-state migration cannot be ignored in any careful analysis of the costs and benefits of migration.

\section{Appendix: GPS robustness checks}

As a first check on the robustness of the results, I carry out the GPS estimation adding the 48 observations corresponding to Mexico City into the sample, resulting in a starting sample size of 1536 state-state relationships, later reduced to 1478 after exclusion 
according to the modified common support condition. If results are indeed robust to the inclusion of the extra Mexico City observations, the potential worries created by this sampling decision are minimized. Tables A.3 to A.7 and Figure A.2 display the key results for the larger sample, exhibiting only minor changes from the tables and figures highlighted previously. The GPS estimation allows for an improvement in balance from only 36\% before the GPS to 76\% after the GPS adjustment of covariate comparisons reflecting a lack of statistically significant differences at the $5 \%$ level; all but 3 covariate comparisons show lack of statistical significance after both GPS adjustment and exclusion based on the modified common support condition. The dose-response function in Figure A.2 is shifted slightly downward from that displayed in Figure 2.3, due to a slight decrease across the board in marginal exports associated with the marginal migrant. The inclusion of the Mexico City observations results in a dose-response function that corresponds to marginal contributions of $\$ 8118, \$ 1678$, and $\$ 158$, respectively, for the 25th, 50th, and 75th percentiles ( $M=34,228$, and 1094) of the actual state-state migrant stock distribution for the $n=1478$ sample. Furthermore, the downward shift results in an increased range for the exhaustion zone, now made up of migrant stocks between 2033 and 4465, corresponding to $7.4 \%$ of state-state migrant stocks.

Additionally, as detailed above, concerns may exist as to how well the matrícula consular data represents the actual state-state migrant distribution. In turn, I conduct a second check for robustness further excluding all observations associated with Texas and Illinois, the two outlier states from the original sample, resulting in a revised sample of 1426 state-state observations, then reduced to 1380 by exclusion following the modified common support condition. The corresponding dose-response and treatment effect 
functions are highlighted in Figure A.3, once again reflecting slight changes in outcomes from the original generalized propensity scores estimation. The GPS adjustment and use of the modified common support condition again provide a balancing of covariates, improving the percentage lacking statistically significant differences at the $5 \%$ level from $33 \%$ to $87 \%$ to $94 \%$ of all possible covariate comparisons. Without the observations corresponding to Texas and Illinois, the dose-response and treatment effect functions again shift slightly downward, resulting in an exhaustion zone bordered by migrant stocks of 964 and 8845 , corresponding to $21.0 \%$ of state-state migrant stocks. Marginal contributions of $\$ 9238$, \$1641, and $\$ 18$, respectively, correspond to the 25th, 50th, and 75th percentiles ( $M=31,201$, and 914$)$ of the actual state-state migrant stock distribution for the $n=$ 1380 sample.

Table 2.1. Descriptive statistics - base and alternative samples

\begin{tabular}{llll}
\hline Variable & $\begin{array}{l}\text { Base sample } \\
n=1536\end{array}$ & $\begin{array}{l}\text { Base sample minus } \\
\text { Mexico City } n=1488\end{array}$ & $\begin{array}{l}\text { Base sample minus Texas } \\
\text { and Illinois } n=1472\end{array}$ \\
\hline Exports (in USD) 81,993,616 & $73,115,405$ & $50,679,743$ \\
$(674,482,678)$ & $(658,528,331)$ & $(385,569,081)$ \\
& $0 / 17,900,000,000$ & $0 / 17,900,000,00$ & $0 / 11,800,000,000$
\end{tabular}




\begin{tabular}{|c|c|c|c|}
\hline Migration $_{j}^{i}$ & $\begin{array}{l}3038 \\
(13,771) \\
0 / 270,201\end{array}$ & $\begin{array}{l}2938 \\
(13,634) \\
0 / 270,201\end{array}$ & $\begin{array}{l}2377 \\
(12,786) \\
0 / 270,201\end{array}$ \\
\hline Migration $_{j}^{a d j}$ & 12,409 & 12,070 & 12,770 \\
\hline & $\begin{array}{l}(27,591) \\
1 / 308,918\end{array}$ & $\begin{array}{l}(27,410) \\
1 / 308,918\end{array}$ & $\begin{array}{l}(28,108) \\
1 / 308,918\end{array}$ \\
\hline Migration $_{j}^{r e s t}$ & 130,355 & 126,016 & 130,655 \\
\hline & $\begin{array}{l}(124,937) \\
858 / 525,394\end{array}$ & $\begin{array}{l}(124,375) \\
858 / 525,394\end{array}$ & $\begin{array}{l}(125,141) \\
858 / 525,394\end{array}$ \\
\hline Distance (miles) & $\begin{array}{l}2078 \\
(597) \\
239 / 3681\end{array}$ & $\begin{array}{l}2077 \\
(600) \\
239 / 3681\end{array}$ & $\begin{array}{l}2106 \\
(585) \\
239 / 3681\end{array}$ \\
\hline Adjacency & $\begin{array}{l}0.006 \\
(0.076) \\
0 / 1\end{array}$ & $\begin{array}{l}0.006 \\
(0.077) \\
0 / 1\end{array}$ & $\begin{array}{l}0.004 \\
(0.059) \\
0 / 1\end{array}$ \\
\hline $\begin{array}{l}\text { US GSP } \\
\text { (bn. USD) }\end{array}$ & $\begin{array}{l}34,392.30 \\
(39,909.53) \\
2,950 / 218,967\end{array}$ & $\begin{array}{l}34,392.30 \\
(39,909.53) \\
2,950 / 218,967\end{array}$ & $\begin{array}{l}31,232.81 \\
(37,118.12) \\
2,950 / 218,967\end{array}$ \\
\hline $\begin{array}{l}\text { Mex. GSP } \\
\text { (bn. USD) }\end{array}$ & $\begin{array}{l}2,969.87 \\
(3,197.62) \\
529 / 17,097\end{array}$ & $\begin{array}{l}2,514.13 \\
(1,975.85) \\
529 / 9,235\end{array}$ & $\begin{array}{l}2,969.87 \\
(3,197.62) \\
529 / 17,097\end{array}$ \\
\hline
\end{tabular}

Notes: For each variable, means are listed first, standard deviations are reported in parentheses, while minimum/maximum pairs are reported in italics.

Table 2.2. Coefficient estimates using gravity equation (OLS, state fixed effects) Dependent variable: US-Mexico state-state exports

\begin{tabular}{llllll}
\hline Independent variable & $(1)$ & $(2)$ & $(3)$ & $(4)$ & $(5)$ \\
\hline Migration $_{j}^{i}$ & $0.1134^{* * *}$ & $0.2403^{* * *}$ & $0.1635^{* * *}$ & $0.1372^{* * *}$ & $0.0735^{*}$ \\
& $(0.0382)$ & $(0.0348)$ & $(0.0420)$ & $(0.0426)$ & $(0.0432)$
\end{tabular}




\begin{tabular}{|c|c|c|c|c|c|}
\hline \multicolumn{3}{|l|}{ Migration $_{j}^{a d j}$} & $\begin{array}{l}0.1354 * * * \\
(0.0418)\end{array}$ & $\begin{array}{l}0.0742 \\
(0.0457)\end{array}$ & $\begin{array}{c}0.0814 * \\
(0.0457)\end{array}$ \\
\hline \multicolumn{3}{|l|}{ Migration $_{j}^{r e s t}$} & & $-0.5031 * *$ & $*-0.0215$ \\
\hline & & & & $(0.1539)$ & $(0.1685)$ \\
\hline Distance & $\begin{array}{l}-1.5952^{* * *} \\
(0.2913)\end{array}$ & & & & $\begin{array}{c}-1.4335^{* * *} \\
(0.3177)\end{array}$ \\
\hline Adjacency & $\begin{array}{l}1.2308^{* * *} \\
(0.4449)\end{array}$ & & & & $\begin{array}{l}1.3146^{* * * *} \\
(0.4531)\end{array}$ \\
\hline Economy size & $\begin{array}{l}0.9365^{* * *} \\
(0.1391)\end{array}$ & & & & $\begin{array}{l}1.1589^{* * *} \\
(0.1607)\end{array}$ \\
\hline$R^{2}$ & 0.8192 & 0.8116 & 0.8130 & 0.8143 & 0.8197 \\
\hline$n$ & 1536 & 1536 & 1536 & 1536 & 1536 \\
\hline
\end{tabular}

Notes: Each estimate is from a separate OLS regression with the logarithm of U.S.-Mexico state-state exports in U.S. dollars plus one as the dependent variable, employing the base sample of the 48 contiguous U.S. states and 32 Mexican states. All regressions include state fixed effects, controlling for any existing systematic differences across states that may affect all states' outcomes. Column (1) displays estimates for coefficients corresponding to the initial OLS equation. Column (2) reports estimates using only Migration ${ }_{j}^{i}$ as an explanatory variable, column (3) adds Migration $_{j}^{a d j}$ as an explanatory variable, column (4) adds Migration $_{j}^{\text {rest }}$ and column (5) displays the preferred specification, adding the remainder of the relevant controls. Heteroskedasticity-consistent robust standard errors are reported in parentheses. ${ }^{* * *},{ }^{* *}$ and $* *$ denote statistical significance at the $1 \%, 5 \%$ and $10 \%$ levels, respectively. 
Table 2.3. Comparison of estimates for the elasticity of exports to migration

\begin{tabular}{|c|c|c|c|c|}
\hline Authors & $\begin{array}{l}\text { Elasticity of exports } \\
\text { to migration }\end{array}$ & $\begin{array}{l}\text { Extra annual exports } \\
\text { generated per extra migrant }\end{array}$ & Sample & Specification-Method \\
\hline My estimates & $\begin{array}{l}0.07 \text { (in-state) } \\
0.08 \text { (neighboring-state) }\end{array}$ & $\begin{array}{l}\text { \$1984 (in-state) } \\
\text { \$3792 (across all states) }\end{array}$ & $\begin{array}{l}48 \text { US states, } 32 \\
\text { Mexican states, } \\
2006-10\end{array}$ & $\begin{array}{l}\text { Pooled cross section, OLS with } \\
\text { state-state trading partner fixed } \\
\text { effects }\end{array}$ \\
\hline $\begin{array}{l}\text { Aleksynska } \\
\text { \& Peri (2011) }\end{array}$ & 0.25 & $\$ 24,895$ & $\begin{array}{l}\text { CEPII “square” } \\
\text { gravity data set, } \\
5230 \text { observations }\end{array}$ & $\begin{array}{l}\text { Pooled cross section, OLS with } \\
\text { country-country fixed effects }\end{array}$ \\
\hline APR (2012) & $\begin{array}{l}0.02,0.08,0.07 \\
\text { (in-state) } \\
0.00,0.02,-0.04 \\
\text { (neighboring-state) }\end{array}$ & $\begin{array}{l}--- \\
---\end{array}$ & $\begin{array}{l}\text { Italy, Portugal, and } \\
\text { Spain, “about } 100 \\
\text { countries,” 2002-10 }\end{array}$ & $\begin{array}{l}\text { Panel, OLS with country-time } \\
\text { and trading partner pairs } \\
\text { fixed effects }\end{array}$ \\
\hline $\begin{array}{l}\text { Felbermayr \& } \\
\text { Jung (2009) }\end{array}$ & 0.11 & $\$ 2717$ & $\begin{array}{l}21 \text { “North" countries } \\
\text { and } 114 \text { "South” } \\
\text { countries, } 1988-2000\end{array}$ & $\begin{array}{l}\text { Pooled cross section, OLS, } \\
\text { differenced with country-country } \\
\text { fixed effects }\end{array}$ \\
\hline HS (2005) & $\begin{array}{l}0.16 \text { (in-state) } \\
0.07 \text { (other-state) }\end{array}$ & --- & $\begin{array}{l}50 \text { US states, } 36 \\
\text { countries, } 1993-96\end{array}$ & $\begin{array}{l}\text { Pooled cross section, Tobit and } \\
\text { LAD }\end{array}$ \\
\hline $\begin{array}{l}\text { Peri \& Requeña } \\
\text { (2010) }\end{array}$ & $0.05-0.11$ & --- & $\begin{array}{l}50 \text { Spanish } \\
\text { provinces, } 77 \\
\text { countries, 1993- } \\
2008\end{array}$ & $\begin{array}{l}\text { Panel, OLS, 2SLS with country- } \\
\text { time and trading partner pairs } \\
\text { fixed effects }\end{array}$ \\
\hline White (2007) & 0.11 & $\$ 2608$ & $\begin{array}{l}\text { US, } 73 \text { countries, } \\
1980-2001\end{array}$ & $\begin{array}{l}\text { Pooled cross section, OLS with } \\
\text { country-country fixed effects }\end{array}$ \\
\hline
\end{tabular}


Table 2.4. Coefficient estimates using gravity equation, alternate samples (OLS, state fixed effects)

\begin{tabular}{|c|c|c|c|c|c|c|c|c|}
\hline \multicolumn{9}{|c|}{ Dependent variable: US-Mexico state-state exports, $n=1488$ and $n=1472$} \\
\hline Independent variable & & (1) & $(2$ & & (3) & & (4) & \\
\hline \multirow[t]{2}{*}{ Migration $_{j}^{i}$} & $0.2394 * * *$ & $0.2349 * * *$ & $0.1616^{* * *}$ & $0.1554 * * *$ & $0.1351 * * *$ & $0.1355 * * *$ & 0.0671 & $0.0764 *$ \\
\hline & $(0.0354)$ & $(0.0360)$ & $(0.0428)$ & $(0.0436)$ & $(0.0434)$ & $(0.0440)$ & $(0.0440)$ & $(0.0444)$ \\
\hline \multirow[t]{2}{*}{$\operatorname{Migration}_{j}^{a d j}$} & & & $0.1382 * * *$ & $0.1371^{* * *}$ & 0.0748 & 0.0725 & $0.0782 *$ & 0.0767 \\
\hline & & & $(0.0431)$ & $(0.0429)$ & $(0.0472)$ & $(0.0475)$ & $(0.0471)$ & $(0.0476)$ \\
\hline \multirow[t]{2}{*}{ Migration $_{j}^{r e s t}$} & & & & & $-0.5101 * *$ & $*-0.5032 * *$ & $*-0.0058$ & -0.0653 \\
\hline & & & & & (0.1565) & $(0.1608)$ & $(0.1713)$ & $(0.1730)$ \\
\hline \multirow[t]{2}{*}{ Distance } & & & & & & & $-1.5813^{* * *}$ & $*-1.3860 * * *$ \\
\hline & & & & & & & $(0.3289)$ & $(0.3351)$ \\
\hline \multirow[t]{2}{*}{ Adjacency } & & & & & & & $1.2479 * * *$ & $1.7183^{* * *}$ \\
\hline & & & & & & & $(0.4603)$ & $(0.6060)$ \\
\hline \multirow[t]{2}{*}{ Economy size } & & & & & & & $0.9927 * * *$ & $1.1865^{* * *}$ \\
\hline & & & & & & & $(0.1510)$ & $(0.1656)$ \\
\hline$R^{2}$ & 0.7989 & 0.7965 & 0.8004 & 0.7980 & 0.8019 & 0.7994 & 0.8081 & 0.8052 \\
\hline$n$ & 1488 & 1472 & 1488 & 1472 & 1488 & 1472 & 1488 & 1472 \\
\hline
\end{tabular}

Notes: Each estimate is from a separate OLS regression with the logarithm of US-Mexico state-state exports in US dollars plus one as the dependent variable, employing the two alternative samples. All regressions include state fixed effects, controlling for any existing systematic differences across states that may affect all states' outcomes. Column (1) reports estimates using only Migration ${ }_{j}^{i}$ as an explanatory variable, column (2) adds Migration ${ }_{j}^{a d j}$ as an explanatory variable, column (3) adds Migration ${ }_{j}^{\text {rest }}$ and column (4) displays the preferred specification, adding the remainder of the relevant controls. Heteroskedasticityconsistent robust standard errors are reported in parentheses. ${ }^{* * *},{ }^{* *}$ and ${ }^{* *}$ denote statistical significance at the $1 \%, 5 \%$ and $10 \%$ levels, respectively. 
Table 2.5. $\gamma_{1}$ and $\gamma_{2}$ with alternative (in)dependent variables (OLS, state fixed effects)

\begin{tabular}{|c|c|c|c|}
\hline (In)dependent variable & $\begin{array}{l}\text { Base sample } \\
n=1536\end{array}$ & $\begin{array}{l}\text { Base sample minus Mexico } \\
\text { City } n=1488\end{array}$ & $\begin{array}{l}\text { Base sample minus Texas } \\
\text { and Illinois } n=1472\end{array}$ \\
\hline \multicolumn{4}{|c|}{ U.S.-Mexico state-state exports 2008} \\
\hline Migration $_{j}^{i}$ 2006-08 & $0.0929 * *(0.0445)$ & $0.0849 *(0.0454)$ & $0.0935 * *(0.0455)$ \\
\hline Migration $_{j}^{a d j} 2006-08$ & $0.0859 * \quad(0.0484)$ & $0.0850 * \quad(0.0498)$ & $0.0875 * \quad(0.0489)$ \\
\hline$R^{2}$ & 0.8028 & 0.7908 & 0.7991 \\
\hline \multicolumn{4}{|c|}{ U.S.-Mexico state-state exports 2009} \\
\hline Migration $_{j}^{i}$ 2006-09 & $0.0803^{*} \quad(0.0461)$ & $(0.0470)$ & $0.0824 * \quad(0.0470)$ \\
\hline Migration $_{j}^{a d j}$ 2006-09 & $(0.0492)$ & $(0.0508)$ & $(0.0499)$ \\
\hline$R^{2}$ & 0.7940 & 0.7806 & 0.7898 \\
\hline
\end{tabular}


Table 2.6. Coefficient estimates using forwarded exports (OLS, state fixed effects)

\begin{tabular}{|c|c|c|c|c|c|c|}
\hline $\mathrm{J}$ & $(1)$ & $(2)$ & $(3)$ & $(4)$ & $(5)$ & $(6)$ \\
\hline Exports measure & 2008 & 2009 & 2010 & 2009 & 2010 & 2010 \\
\hline Migration measure & 2006-07 & 2006-07 & 2006-07 & 2006-08 & 2006-08 & 2006-09 \\
\hline$\overline{\text { Migration }_{j}^{i}}$ & $\begin{array}{l}0.1006 * * \\
(0.0444)\end{array}$ & $\begin{array}{l}0.1013 * * \\
(0.0446)\end{array}$ & $\begin{array}{l}0.0980 * * \\
(0.0446)\end{array}$ & $\begin{array}{l}0.0922 * * \\
(0.0447)\end{array}$ & $\begin{array}{l}0.0926 * * \\
(0.0447)\end{array}$ & $\begin{array}{l}0.0789 * \\
(0.0461)\end{array}$ \\
\hline Migration $_{j}^{a d j}$ & $\begin{array}{l}0.0824 * \\
(0.0484)\end{array}$ & $\begin{array}{l}0.0473 \\
(0.0486)\end{array}$ & $\begin{array}{l}0.0533 \\
(0.0486)\end{array}$ & $\begin{array}{l}0.0514 \\
(0.0486)\end{array}$ & $\begin{array}{l}0.0558 \\
(0.0486)\end{array}$ & $\begin{array}{l}0.0599 \\
(0.0492)\end{array}$ \\
\hline Migration $_{j}^{\text {rest }}$ & $\begin{array}{l}-0.0938 \\
(0.1809)\end{array}$ & $\begin{array}{l}-0.1593 \\
(0.1818)\end{array}$ & $\begin{array}{l}-0.0024 \\
(0.1816)\end{array}$ & $\begin{array}{l}-0.1614 \\
(0.1818)\end{array}$ & $\begin{array}{l}-0.0035 \\
(0.1816)\end{array}$ & $\begin{array}{l}-0.0083 \\
(0.1817)\end{array}$ \\
\hline Distance & $\begin{array}{l}-1.4556^{* * *} \\
(0.3390)\end{array}$ & $\begin{array}{l}-1.3079 * * * \\
(0.3405)\end{array}$ & $\begin{array}{l}-1.6222^{* * *} \\
(0.3402)\end{array}$ & $\begin{array}{l}-1.3146^{* * *} \\
(0.3410)\end{array}$ & $\begin{array}{l}-1.6242^{* * *} \\
(0.3407)\end{array}$ & $\begin{array}{l}-1.6367 * * * \\
(0.3417)\end{array}$ \\
\hline Adjacency & $\begin{array}{l}1.3076 * * * \\
(0.4866)\end{array}$ & $\begin{array}{l}1.2909 * * * \\
(0.4889)\end{array}$ & $\begin{array}{l}0.9733 * * * \\
(0.4884)\end{array}$ & $\begin{array}{l}1.2990 * * * \\
(0.4889)\end{array}$ & $\begin{array}{l}0.9791 * * * \\
(0.4884)\end{array}$ & $\begin{array}{l}0.9907 * * * \\
(0.4886)\end{array}$ \\
\hline Economy size & $\begin{array}{l}1.3044 * * * \\
(0.1723)\end{array}$ & $\begin{array}{l}1.2673 * * * \\
(0.1731)\end{array}$ & $\begin{array}{l}1.1223 * * * \\
(0.1729)\end{array}$ & $\begin{array}{l}1.2717 * * * \\
(0.1733)\end{array}$ & $\begin{array}{l}1.1242 * * * \\
(0.1731)\end{array}$ & $\begin{array}{l}1.1360 * * * \\
(0.1731)\end{array}$ \\
\hline$R^{2}$ & 0.8029 & 0.7943 & 0.8076 & 0.7941 & 0.8075 & 0.8073 \\
\hline$n$ & 1536 & 1536 & 1536 & 1536 & 1536 & 1536 \\
\hline
\end{tabular}

Notes: Each estimate is from a separate OLS regression with the logarithm of U.S.-Mexico state-state exports in U.S. dollars plus one as the dependent variable, employing the base sample of the 48 contiguous U.S. states and 32 Mexican states. All regressions include state fixed effects, controlling for any existing systematic differences across states that may affect all states' outcomes. Heteroskedasticity-consistent robust standard errors are reported in parentheses. $* * *$, ** and ** denote statistical significance at the $1 \%, 5 \%$ and $10 \%$ levels, respectively. 
Table 2.7. Coefficient estimates using gravity equation, migrant share (OLS, state fixed effects)

\begin{tabular}{llll}
\hline & Base sample & $\begin{array}{l}\text { Base sample minus } \\
\text { Mexico City } \\
n=1488\end{array}$ & $\begin{array}{l}\text { Base sample minus } \\
\text { Texas and Illinois } \\
n=1472\end{array}$ \\
Independent variable & $n=1536$ & $0.1172^{*}$ & $0.1397^{* *}$ \\
\hline Migrant share ${ }_{j}^{i}$ & $0.1251^{* *}$ & $(0.0631)$ & $(0.0636)$ \\
Distance & $(0.0620)$ & $-1.8887^{* * *}$ & $-1.7565^{* * *}$ \\
& $-1.7645^{* * *}$ & $(0.2893)$ & $(0.2958)$ \\
Adjacency & $(0.2815)$ & $1.1264^{* *}$ & $1.5184^{* *}$ \\
& $1.2013^{* * *}$ & $(0.4529)$ & $(0.5984)$ \\
Economy size & $(0.4461)$ & $0.6784^{* * *}$ & $1.1359^{* * *}$ \\
& $1.2147^{* * *}$ & $(0.1718)$ & $(0.1204)$ \\
$R^{2}$ & $(0.0907)$ & 0.8072 & 0.8041 \\
$n$ & 0.8186 & 1488 & 1472 \\
\hline
\end{tabular}

Notes: Each column of estimates is from a separate OLS regression with the logarithm of U.S.-Mexico statestate exports in U.S. dollars plus one as the dependent variable, employing the two alternative samples. All regressions include state fixed effects, controlling for any existing systematic differences across states that may affect all states' outcomes. Heteroskedasticity-consistent robust standard errors are reported in parentheses. ${ }^{* *},{ }^{* *}$ and ${ }^{* *}$ denote statistical significance at the $1 \%, 5 \%$ and $10 \%$ levels, respectively.

Table 2.8. OLS estimation, dependent variable: $\ln$ (migrants) 


\begin{tabular}{|c|c|c|}
\hline Covariates and statistics & Estimated coefficient & Standard error \\
\hline$\overline{\text { U.S. population }}$ & $20.31 * * *$ & 4.18 \\
\hline U.S. population ${ }^{2}$ & $-0.75 * * *$ & 0.14 \\
\hline Mex. Population & $-5.82 * *$ & 2.28 \\
\hline Mex. population ${ }^{2}$ & $0.26 * * *$ & 0.08 \\
\hline U.S. GSP & $-7.46^{* *}$ & 3.14 \\
\hline U.S. GSP ${ }^{2}$ & $0.48^{* * *}$ & 0.14 \\
\hline Mex. GSP & -0.03 & 1.75 \\
\hline Mex. GSP ${ }^{2}$ & -0.05 & 0.09 \\
\hline U.S. pop. growth & $6.58^{* * *}$ & 0.44 \\
\hline Mex. pop. growth & $-7.12 * * *$ & 0.75 \\
\hline U.S. GSP growth & $2.11^{* * *}$ & 0.68 \\
\hline Mex. GSP growth & $2.05^{* * *}$ & 0.46 \\
\hline Adjacency & -0.17 & 0.46 \\
\hline Distance & $-3.00 * * *$ & 0.14 \\
\hline U.S. unemployment & $0.32 * * *$ & 0.04 \\
\hline Mex. unemployment & $0.10^{* *}$ & 0.04 \\
\hline U.S. Gini & $-33.60 * * *$ & 2.38 \\
\hline Mex. Gini & $19.86 * * *$ & 13.32 \\
\hline Constant & $-47.89 * * *$ & 16.70 \\
\hline Obervations & 1488 & \\
\hline$R^{2}$ & 0.76 & \\
\hline
\end{tabular}


Table 2.9. Groups and blocks, full sample $n=1488$

\begin{tabular}{lllllllll}
\hline Block & Group 1 & Control 1 & Group 2 & Control 2 & Group 3 & Control 3 & Group 4 & Control 4 \\
\hline 1 & 37 & 958 & 37 & 651 & 37 & 565 & 37 & 738 \\
2 & 37 & 64 & 37 & 108 & 37 & 122 & 37 & 123 \\
3 & 38 & 30 & 37 & 65 & 37 & 76 & 37 & 100 \\
4 & 37 & 17 & 37 & 81 & 38 & 72 & 37 & 47 \\
5 & 38 & 18 & 37 & 54 & 37 & 71 & 37 & 25 \\
6 & 37 & 14 & 38 & 22 & 37 & 73 & 37 & 15 \\
7 & 37 & 5 & 37 & 30 & 38 & 44 & 37 & 13 \\
8 & 38 & 2 & 37 & 52 & 37 & 29 & 37 & 13 \\
9 & 37 & 4 & 37 & 31 & 37 & 41 & 37 & 14 \\
10 & 37 & 3 & 37 & 23 & 37 & 23 & & \\
\hline
\end{tabular}

Table 2.10. Groups and blocks, modified common support sample $n=1429$

\begin{tabular}{lllllllll}
\hline Block & Group 1 & Control 1 & Group 2 & Control 2 & Group 3 & Control 3 & Group 4 & Control 4 \\
\hline 1 & 33 & 944 & 37 & 592 & 37 & 506 & 35 & 742 \\
2 & 34 & 62 & 37 & 108 & 37 & 122 & 35 & 132 \\
3 & 33 & 29 & 37 & 65 & 37 & 76 & 35 & 78 \\
4 & 34 & 26 & 37 & 81 & 38 & 72 & 35 & 28 \\
5 & 34 & 12 & 37 & 54 & 37 & 71 & 35 & 27 \\
6 & 33 & 10 & 38 & 22 & 37 & 73 & 35 & 19 \\
7 & 34 & 3 & 37 & 30 & 38 & 44 & 35 & 14 \\
8 & 33 & 1 & 37 & 52 & 37 & 29 & 35 & 12 \\
9 & 34 & 5 & 37 & 31 & 37 & 41 & 35 & 14 \\
10 & 33 & 2 & 37 & 23 & 37 & 23 & & 35 \\
\hline
\end{tabular}


Table 2.11. Three-stage balancing comparison of covariates

\begin{tabular}{|c|c|c|c|c|c|c|c|c|c|c|c|c|}
\hline \multirow{2}{*}{$\frac{\text { T-stats }}{\text { Covariate }}$} & \multicolumn{4}{|c|}{$\begin{array}{l}\text { Before balancing on GPS } \\
n=1488\end{array}$} & \multicolumn{4}{|c|}{$\begin{array}{l}\text { After balancing on GPS } \\
\text { (weighted t-stats) } n=1488\end{array}$} & \multicolumn{4}{|c|}{$\begin{array}{l}\text { Common support after balancing } \\
\text { GPS (weighted t-stats) } n=1429\end{array}$} \\
\hline & Q1 & Q2 & Q3 & Q4 & Q1 & Q2 & Q3 & Q4 & Q1 & Q2 & Q3 & Q4 \\
\hline U.S. population & 21.72 & 0.12 & -4.04 & -16.19 & 2.52 & -0.41 & 0.41 & -3.49 & 0.83 & -0.30 & 0.42 & -1.28 \\
\hline U.S. population ${ }^{2}$ & 21.19 & 0.29 & -3.69 & -16.37 & 2.39 & -0.40 & 0.46 & -3.62 & 0.79 & -0.30 & 0.47 & -1.26 \\
\hline Mex. Population & 9.45 & 4.74 & -2.67 & -11.69 & 2.25 & 1.41 & -0.25 & -2.65 & 1.21 & 1.58 & -0.18 & -1.82 \\
\hline Mex. population ${ }^{2}$ & 9.30 & 4.80 & -2.55 & -11.74 & 2.17 & 1.42 & -0.23 & -2.69 & 1.17 & 1.57 & -0.16 & -1.86 \\
\hline U.S. GSP & 21.39 & 0.18 & -3.63 & -16.50 & 2.30 & -0.42 & 0.41 & -3.65 & 0.61 & -0.30 & 0.44 & -1.35 \\
\hline U.S. GSP ${ }^{2}$ & 20.56 & 0.41 & -3.15 & -16.66 & 2.11 & -0.41 & 0.50 & -3.79 & 0.56 & -0.31 & 0.51 & -1.33 \\
\hline Mex. GSP & 5.40 & 0.08 & -0.39 & -5.07 & 1.35 & 0.32 & 0.39 & -1.10 & 0.96 & 0.36 & 0.41 & -0.36 \\
\hline Mex. GSP ${ }^{2}$ & 5.26 & 0.08 & -0.31 & -5.02 & 1.31 & 0.30 & 0.40 & -1.13 & 0.94 & 0.34 & 0.42 & -0.38 \\
\hline U.S. pop. growth & 6.52 & 2.16 & -2.22 & -6.46 & 1.51 & 0.81 & -0.23 & -2.11 & 1.27 & 0.72 & -0.28 & -1.43 \\
\hline Mex. pop. growth & -2.87 & 2.67 & 0.27 & -0.07 & 0.36 & 0.57 & 0.21 & -0.28 & 0.51 & 0.55 & 0.19 & -0.50 \\
\hline U.S. GSP growth & -6.76 & -0.12 & 3.64 & 3.18 & 0.10 & 0.77 & 0.92 & -0.08 & 0.04 & 0.66 & 0.85 & 0.24 \\
\hline Mex. GSP growth & -0.88 & 1.08 & 1.61 & -1.78 & -0.29 & 0.44 & 0.81 & 0.31 & -0.32 & 0.45 & 0.82 & 0.15 \\
\hline Adjacency & 1.74 & 1.73 & 0.96 & -4.48 & -0.59 & 0.73 & 0.41 & -6.25 & 0.26 & 0.34 & -0.32 & 0.13 \\
\hline Distance & -14.03 & -0.47 & 7.18 & 6.72 & -4.75 & -0.47 & 1.62 & 4.14 & -4.18 & -0.45 & 1.95 & 0.20 \\
\hline U.S. unemployment & 10.04 & -0.10 & -2.43 & -7.26 & 1.03 & 0.32 & 0.55 & -0.17 & 0.42 & 0.42 & 0.60 & -0.34 \\
\hline Mex. unemployment & 1.31 & -1.67 & -3.10 & 3.41 & 1.62 & 0.01 & -0.70 & 0.55 & 1.51 & 0.00 & -0.66 & 0.91 \\
\hline U.S. Gini & 9.40 & -1.23 & -1.75 & -6.19 & 1.37 & -0.31 & 0.47 & -1.91 & 0.32 & -0.26 & 0.46 & -0.59 \\
\hline Mex. Gini & 6.18 & 4.31 & -3.50 & -7.00 & 1.46 & 0.40 & -0.87 & -0.68 & 1.16 & 0.53 & -0.83 & -0.52 \\
\hline Avg. absolute t-stat & \multicolumn{4}{|c|}{$5.46,24 / 72<|1.96|$} & \multicolumn{4}{|c|}{$1.22,57 / 72<|1.96|$} & \multicolumn{4}{|c|}{$0.71,71 / 72<|1.96|$} \\
\hline
\end{tabular}


Table 2.12. Covariate means: included vs. excluded observations

\begin{tabular}{lll}
\hline Covariates & $\begin{array}{l}\text { Mean of common } \\
\text { support observations }\end{array}$ & $\begin{array}{l}\text { Mean of excluded } \\
\text { observations }\end{array}$ \\
\hline U.S. population & 15.14 & 15.18 \\
Mex. population & 14.50 & 14.70 \\
U.S. GSP & 12.04 & 12.09 \\
Mex. GSP & 9.77 & 9.86 \\
U.S. pop. growth & 0.03 & 0.03 \\
Mex. pop. growth & 0.09 & 0.09 \\
U.S. GSP growth & 0.07 & 0.07 \\
Mex. GSP growth & 0.08 & 0.08 \\
Adjacency & 0.00 & 0.01 \\
Distance & 7.58 & 7.60 \\
U.S. unemployment & 5.66 & 5.67 \\
Mex. unemployment & 3.82 & 3.77 \\
U.S. Gini & 0.45 & 0.45 \\
Mex. Gini & 0.47 & 0.48 \\
\hline
\end{tabular}

Table 2.13. OLS estimation, dependent variable: $\ln$ (exports)

\begin{tabular}{|c|c|c|}
\hline Covariates and statistics & Estimated coefficient & Standard error \\
\hline Migrant stock & $1.06 * * *$ & 0.30 \\
\hline Migrant stock ${ }^{2}$ & $-0.18 * * *$ & 0.07 \\
\hline Migrant stock ${ }^{3}$ & $0.01 * * *$ & 0.00 \\
\hline GPS & $-5.65 * * *$ & 2.54 \\
\hline Migrant stock $\times$ GPS & $1.54 * * *$ & 0.44 \\
\hline Constant & $11.75 * * *$ & 0.60 \\
\hline Observations & 1429 & \\
\hline$R^{2}$ & 0.11 & \\
\hline
\end{tabular}


Table A.1. Matrículas consulares registered 2006 to 2010, U.S. states of residence and Mexican states of origin: total of 4,659,656

\begin{tabular}{lll}
\hline & U.S. & \\
\hline Alabama 27,442 & Oklahoma 19,867 & Mexico \\
Arizona 187,032 & Oregon 74,103 & Baja California 45,183 \\
Arkansas 19,711 & Pennsylvania 23,555 & Baja California Sur 3,178 \\
California 1,682,667 & Rhode Island 931 & Campeche 7,017 \\
Colorado 105,125 & South Carolina 38,551 & Chiapas 62,697 \\
Connecticut 10,645 & South Dakota 723 & Chihuahua 120,933 \\
Delaware 6,637 & Tennessee 38,736 & Coahuila 56,687 \\
Florida 107,392 & Texas 779,636 & Colima 32,326 \\
Georgia 150,704 & Utah 55,330 & Durango 126,923 \\
Idaho 16,340 & Vermont 345 & Guanajuato 377,674 \\
Illinois 387,377 & Virginia 24,492 & Guerrero 371,279 \\
Indiana 69,247 & Washington 64,436 & Hidalgo 131,280 \\
Iowa 15,953 & West Virginia 663 & Jalisco 425,607 \\
Kansas 21,981 & Wisconsin 43,532 & Mexico 238,343 \\
Kentucky 14,428 & Wyoming 3,563 & Mexico City 293,920 \\
Louisiana 8,074 & & Michoacan 525,514 \\
Maine 232 & & Morelos 105,732 \\
Maryland 20,729 & & Nayarit 72,227 \\
Massachusetts 2,629 & & Nuevo Leon 77,824 \\
Michigan 22,417 & & Oaxaca 283,295 \\
Minnesota 38,019 & & Puebla 307,606 \\
Missouri 17,103 & & Queretaro 58,608 \\
Mississippi 5,600 & & Quintana Roo 3,470 \\
Montana 164 & San Luis Potosi 155,069 \\
Nebraska 22,291 & Sinaloa 91,019 \\
New Hampshire 854 & Sonora 49,074 \\
New Jersey 73,881 & & Tabasco 72,502 \\
New Mexico 53,212 & & Tamaulipas 98,290 \\
New York 133,625 & & Veracruz 205,799 \\
Nevada 108,310 & & Yucatan 17,837 \\
North Carolina 142,813 & & \\
North Dakota 54 & & \\
Ohio 18,505 & & \\
\hline
\end{tabular}


Table A.2. Migration and trade in top U.S. states of Mexican migrant residence

\begin{tabular}{|c|c|c|c|c|}
\hline \multicolumn{3}{|c|}{ California } & \multicolumn{2}{|l|}{ Texas } \\
\hline Rank & Migration & Exports & Migration & Exports \\
\hline 1 & Michoacan & Baja California & Guanajuato & Chihuahua \\
\hline 2 & Jalisco & Mexico & San Luis Potosi & Tamaulipas \\
\hline 3 & Guerrero & Chihuahua & Tamaulipas & Mexico City \\
\hline 4 & Оахаса & Mexico City & Nuevo Leon & Mexico \\
\hline 5 & Mexico City & Jalisco & Michoacan & Coahuila \\
\hline 6 & Guanajuato & Sonora & Guerrero & Nuevo Leon \\
\hline 7 & Puebla & Nuevo Leon & Zacatecas & Guanajuato \\
\hline 8 & Mexico & Sinaloa & Mexico & Jalisco \\
\hline 9 & Zacatecas & Tamaulipas & Coahuila & Queretaro \\
\hline 10 & Sinaloa & Puebla & Mexico City & Aguascalientes \\
\hline 11 & Nayarit & Baja California Sur & Veracruz & San Luis Potosi \\
\hline 12 & Veracruz & Queretaro & Jalisco & Veracruz \\
\hline 13 & Morelos & Guanajuato & Durango & Hidalgo \\
\hline 14 & Hidalgo & Coahuila & Chihuahua & Sonora \\
\hline 15 & Baja California & Aguascalientes & Hidalgo & Durango \\
\hline 16 & Durango & Durango & Puebla & Tabasco \\
\hline 17 & Tabasco & San Luis Potosi & Queretaro & Baja California \\
\hline 18 & Colima & Quintana Roo & Оахаса & Puebla \\
\hline 19 & Queretaro & Tlaxcala & Morelos & Michoacan \\
\hline 20 & Chiapas & Veracruz & Aguascalientes & Sinaloa \\
\hline 21 & Sonora & Hidalgo & Tabasco & Morelos \\
\hline 22 & Yucatan & Michoacan & Chiapas & Quintana Roo \\
\hline 23 & Aguascalientes & Morelos & Tlaxcala & Campeche \\
\hline 24 & Tlaxcala & Yucatan & Sinaloa & Colima \\
\hline 25 & Chihuahua & Campeche & Colima & Zacatecas \\
\hline 26 & San Luis Potosi & Nayarit & Nayarit & Tlaxcala \\
\hline 27 & Coahuila & Tabasco & Campeche & Yucatan \\
\hline 28 & Tamaulipas & Zacatecas & Baja California & Оахаса \\
\hline 29 & Nuevo Leon & Chiapas & Yucatan & Chiapas \\
\hline 30 & Campeche & Colima & Sonora & Baja California \\
\hline 31 & Quintana Roo & Оахаса & Quintana Roo & Guerrero \\
\hline 32 & Baja California Sur & Guerrero & Baja California Sur & Nayarit \\
\hline
\end{tabular}


Table A.3. OLS estimation, dependent variable: $\ln$ (migrants)

\begin{tabular}{|c|c|c|}
\hline Covariates and statistics & Estimated coefficient & Standard error \\
\hline U.S. population & $19.33 * * *$ & 4.09 \\
\hline U.S. population ${ }^{2}$ & $-0.72 * * *$ & 0.14 \\
\hline Mex. Population & -2.73 & 1.89 \\
\hline Mex. population ${ }^{2}$ & $0.15^{* *}$ & 0.06 \\
\hline U.S. GSP & $-6.61^{* *}$ & 3.08 \\
\hline U.S. GSP ${ }^{2}$ & $0.44^{* * *}$ & 0.13 \\
\hline Mex. GSP & $-3.39 * * *$ & 1.05 \\
\hline Mex. GSP ${ }^{2}$ & $0.12 * *$ & 0.05 \\
\hline U.S. pop. growth & $6.62 * * *$ & 0.43 \\
\hline Mex. pop. growth & $-6.84 * * *$ & 0.74 \\
\hline U.S. GSP growth & $2.20 * * *$ & 0.66 \\
\hline Mex. GSP growth & $1.94^{* * *}$ & 0.45 \\
\hline Adjacency & -0.08 & 0.45 \\
\hline Distance & $-2.92 * * *$ & 0.14 \\
\hline U.S. unemployment & $0.32 * * *$ & 0.04 \\
\hline Mex. unemployment & $0.10 * *$ & 0.04 \\
\hline U.S. Gini & $-33.02 * * *$ & 2.33 \\
\hline Mex. Gini & $20.95 * * *$ & 1.41 \\
\hline Constant & $-53.49 * * *$ & 16.32 \\
\hline Obervations & 1536 & \\
\hline$R^{2}$ & 0.77 & \\
\hline
\end{tabular}


Table A.4. Groups and blocks, full sample $n=1536$

\begin{tabular}{lllllllll}
\hline Block & Group 1 & Control 1 & Group 2 & Control 2 & Group 3 & Control 3 & Group 4 & Control 4 \\
\hline 1 & 38 & 994 & 38 & 643 & 38 & 592 & 38 & 777 \\
2 & 39 & 57 & 38 & 130 & 38 & 126 & 38 & 114 \\
3 & 39 & 38 & 39 & 68 & 39 & 65 & 39 & 107 \\
4 & 38 & 17 & 38 & 88 & 38 & 83 & 39 & 44 \\
5 & 39 & 16 & 38 & 46 & 38 & 66 & 38 & 29 \\
6 & 39 & 15 & 39 & 43 & 39 & 62 & 38 & 26 \\
7 & 38 & 5 & 38 & 26 & 38 & 45 & 39 & 19 \\
8 & 39 & 2 & 39 & 52 & 39 & 50 & 39 & 11 \\
9 & 39 & 3 & 38 & 29 & 38 & 38 & 38 & 9 \\
10 & 38 & 3 & 38 & 28 & 38 & 26 & 38 & 16 \\
\hline
\end{tabular}

Table A.5. Groups and blocks, modified common support sample $n=1478$

\begin{tabular}{lllllllll}
\hline Block & Group 1 & Control 1 & Group 2 & Control 2 & Group 3 & Control 3 & Group 4 & Control 4 \\
\hline 1 & 34 & 977 & 38 & 593 & 38 & 534 & 36 & 772 \\
2 & 35 & 58 & 38 & 124 & 38 & 126 & 37 & 144 \\
3 & 35 & 35 & 38 & 67 & 39 & 65 & 36 & 57 \\
4 & 36 & 26 & 39 & 89 & 38 & 83 & 37 & 40 \\
5 & 35 & 10 & 38 & 45 & 38 & 66 & 36 & 27 \\
6 & 35 & 10 & 38 & 43 & 39 & 62 & 36 & 27 \\
7 & 34 & 6 & 39 & 26 & 38 & 45 & 37 & 12 \\
8 & 36 & 1 & 38 & 52 & 39 & 50 & 36 & \\
9 & 34 & 3 & 38 & 29 & 38 & 38 & 36 & 16 \\
10 & 35 & 3 & 38 & 28 & 38 & 26 & 36 & \\
\hline
\end{tabular}


Table A.6. Three-stage balancing comparison of covariates

\begin{tabular}{|c|c|c|c|c|c|c|c|c|c|c|c|c|}
\hline \multirow{2}{*}{$\frac{\text { T-stats }}{\text { Covariate }}$} & \multicolumn{4}{|c|}{$\begin{array}{l}\text { Before balancing on GPS } \\
n=1536\end{array}$} & \multicolumn{4}{|c|}{$\begin{array}{l}\text { After balancing on GPS } \\
\text { (weighted t-stats) } n=1536\end{array}$} & \multicolumn{4}{|c|}{$\begin{array}{l}\text { Common support after balancing } \\
\text { GPS (weighted t-stats) } n=1478\end{array}$} \\
\hline & Q1 & $\mathrm{Q} 2$ & Q3 & Q4 & Q1 & $\mathrm{Q} 2$ & Q3 & Q4 & Q1 & Q2 & Q3 & $\mathrm{Q} 4$ \\
\hline U.S. population & 22.15 & 0.02 & -3.60 & -17.21 & 2.66 & -0.27 & 0.59 & -4.05 & 1.07 & -0.35 & 0.61 & -1.93 \\
\hline U.S. population ${ }^{2}$ & 21.63 & 0.19 & -3.26 & -17.41 & 2.52 & -0.25 & 0.64 & -4.19 & 1.03 & -0.34 & 0.65 & -1.93 \\
\hline Mex. Population & 9.84 & 5.53 & -3.38 & -12.23 & 2.22 & 1.48 & -0.57 & -2.45 & 1.24 & 1.62 & -0.48 & -1.61 \\
\hline Mex. population ${ }^{2}$ & 9.70 & 5.61 & -3.27 & -12.29 & 2.15 & 1.50 & -0.57 & -2.48 & 1.20 & 1.64 & -0.48 & -1.63 \\
\hline U.S. GSP & 21.90 & 0.05 & -3.18 & -17.56 & 2.39 & -0.26 & 0.60 & -4.19 & 0.80 & -0.35 & 0.63 & -2.01 \\
\hline U.S. GSP ${ }^{2}$ & 21.05 & 0.29 & -2.70 & -17.75 & 2.20 & -0.23 & 0.68 & -4.35 & 0.74 & -0.33 & 0.70 & -2.01 \\
\hline Mex. GSP & 5.73 & 1.71 & -1.03 & -6.44 & 1.08 & 0.70 & 0.09 & -1.13 & 0.69 & 0.75 & 0.13 & -0.37 \\
\hline Mex. GSP ${ }^{2}$ & 5.59 & 1.80 & -0.97 & -6.46 & 0.97 & 0.70 & 0.09 & -1.15 & 0.66 & 0.76 & 0.12 & -0.40 \\
\hline U.S. pop. growth & 6.26 & 2.13 & -2.08 & -6.32 & 1.40 & 0.83 & 0.03 & -2.02 & 1.19 & 0.75 & -0.05 & -0.88 \\
\hline Mex. pop. growth & -3.03 & 1.47 & 0.75 & 0.81 & 0.35 & -0.20 & 0.40 & -0.27 & 0.53 & -0.21 & 0.37 & -0.31 \\
\hline U.S. GSP growth & -6.91 & 0.47 & 2.87 & 3.50 & 0.05 & -0.93 & 0.86 & -0.04 & 0.09 & 0.78 & 0.78 & 0.37 \\
\hline Mex. GSP growth & -0.93 & 0.62 & 1.71 & -1.39 & -0.41 & 0.55 & 0.85 & 0.24 & -0.45 & 0.81 & 0.85 & 0.12 \\
\hline Adjacency & 1.74 & 1.73 & 0.96 & -4.47 & 0.59 & 0.72 & 0.37 & -6.34 & 0.32 & 0.42 & -0.04 & 0.00 \\
\hline Distance & -14.13 & 0.04 & 7.17 & 6.31 & -4.54 & 0.09 & 1.37 & 4.16 & -3.92 & 0.25 & 1.64 & 0.02 \\
\hline U.S. unemployment & 10.37 & -0.25 & -2.23 & -7.71 & 1.13 & 0.31 & 0.36 & -0.50 & 0.58 & 0.36 & 0.43 & -0.47 \\
\hline Mex. unemployment & 1.81 & -0.63 & -2.72 & 1.54 & 1.28 & 0.10 & -0.55 & 0.21 & 1.15 & 0.12 & -0.51 & 0.52 \\
\hline U.S. Gini & 9.74 & -1.24 & -1.56 & -6.77 & 1.34 & 0.08 & 0.84 & -2.51 & 0.45 & -0.13 & 0.82 & -0.99 \\
\hline Mex. Gini & 6.48 & 4.38 & -4.11 & -6.76 & 1.83 & 0.48 & -1.00 & -0.42 & 1.50 & 0.68 & -0.93 & -0.65 \\
\hline Avg. absolute t-stat & \multicolumn{4}{|c|}{$5.66,26 / 72<|1.96|$} & \multicolumn{4}{|c|}{$1.28,55 / 72<|1.96|$} & \multicolumn{4}{|c|}{$0.76,69 / 72<|1.96|$} \\
\hline
\end{tabular}


Table A.7. OLS estimation, dependent variable: $\ln$ (exports)

\begin{tabular}{lll}
\hline Covariates and statistics & Estimated coefficient & Standard error \\
\hline Migrant stock & $1.11^{* * *}$ & 0.30 \\
Migrant stock $^{2}$ & $-0.21^{* * *}$ & 0.07 \\
Migrant stock & & 0.00 \\
GPS & $0.01^{* * *}$ & 2.50 \\
Migrant stock $\times$ GPS & $-7.58^{* * *}$ & 0.43 \\
Constant & $1.91^{* * *}$ & 0.59 \\
\hline Observations & $12.03^{* * *}$ & \\
$R^{2}$ & & \\
\hline
\end{tabular}

Table A.8. OLS estimation, dependent variable: $\ln$ (migrants)

\begin{tabular}{lll}
\hline Covariates and statistics & Estimated coefficient & Standard error \\
\hline U.S. population & $20.11^{* * *}$ & 4.16 \\
U.S. population ${ }^{2}$ & $-0.75^{* * *}$ & 0.14 \\
Mex. Population & $-6.46^{* * *}$ & 2.31 \\
Mex. population ${ }^{2}$ & $0.28^{* * *}$ & 0.08 \\
U.S. GSP & $-8.01^{* * *}$ & 3.15 \\
U.S. GSP & $0.50^{* * *}$ & 0.14 \\
Mex. GSP & 0.11 & 1.77 \\
Mex. GSP & -0.06 & 0.09 \\
U.S. pop. growth & $6.71^{* * *}$ & 0.44 \\
Mex. pop. growth & $-7.11^{* * *}$ & 0.76 \\
U.S. GSP growth & $2.14^{* * *}$ & 0.67 \\
Mex. GSP growth & $2.04^{* * *}$ & 0.46 \\
Adjacency & -0.13 & 0.60 \\
Distance & $-3.15^{* * *}$ & 0.15 \\
U.S. unemployment & $0.320^{* *}$ & 0.04 \\
Mex. unemployment & $0.08^{* *}$ & 0.04 \\
U.S. Gini & $-33.48^{* * *}$ & 2.36 \\
Mex. Gini & $20.35^{* * *}$ & 1.51 \\
Constant & $-38.22^{* * *}$ & 16.78 \\
\hline Obervations & & \\
$R^{2}$ & & \\
\hline
\end{tabular}


Table A.9. Groups and blocks, full sample $n=1426$

\begin{tabular}{lllllllll}
\hline Block & Group 1 & Control 1 & Group 2 & Control 2 & Group 3 & Control 3 & Group 4 & Control 4 \\
\hline 1 & 36 & 910 & 35 & 614 & 35 & 521 & 35 & 752 \\
2 & 36 & 51 & 35 & 90 & 36 & 125 & 36 & 97 \\
3 & 37 & 33 & 35 & 65 & 36 & 72 & 36 & 90 \\
4 & 36 & 17 & 35 & 63 & 36 & 55 & 35 & 42 \\
5 & 37 & 22 & 35 & 64 & 35 & 82 & 36 & 29 \\
6 & 36 & 15 & 35 & 34 & 36 & 61 & 36 & 25 \\
7 & 36 & 6 & 35 & 39 & 36 & 41 & 35 & 22 \\
8 & 37 & 2 & 35 & 35 & 36 & 47 & 36 & 7 \\
9 & 36 & 5 & 35 & 41 & 36 & 24 & 36 & 35 \\
10 & 36 & 2 & 35 & 31 & 35 & 41 & & 0 \\
\hline
\end{tabular}

Table A.10. Groups and blocks, modified common support sample $\boldsymbol{n}=\mathbf{1 3 8 0}$

\begin{tabular}{lllllllll}
\hline Block & Group 1 & Control 1 & Group 2 & Control 2 & Group 3 & Control 3 & Group 4 & Control 4 \\
\hline 1 & 32 & 904 & 34 & 576 & 35 & 475 & 34 & 709 \\
2 & 33 & 59 & 35 & 88 & 36 & 125 & 35 & 102 \\
3 & 33 & 28 & 36 & 61 & 36 & 72 & 35 & 88 \\
4 & 33 & 22 & 34 & 65 & 36 & 55 & 35 & 42 \\
5 & 33 & 16 & 36 & 61 & 35 & 82 & 35 & 31 \\
6 & 32 & 12 & 35 & 34 & 36 & 61 & 35 & 18 \\
7 & 33 & 4 & 35 & 40 & 36 & 41 & 35 & 8 \\
8 & 33 & 1 & 34 & 34 & 36 & 47 & 35 & \\
9 & 33 & 5 & 36 & 41 & 36 & 24 & 41 & 34 \\
10 & 32 & 2 & 34 & 31 & 35 & & & \\
\hline
\end{tabular}


Table A.11. Three-stage balancing comparison of covariates

\begin{tabular}{|c|c|c|c|c|c|c|c|c|c|c|c|c|}
\hline \multirow{2}{*}{$\frac{\text { T-stats }}{\text { Covariate }}$} & \multicolumn{4}{|c|}{$\begin{array}{l}\text { Before balancing on GPS } \\
n=1426\end{array}$} & \multicolumn{4}{|c|}{$\begin{array}{l}\text { After balancing on GPS } \\
\text { (weighted t-stats) } n=1426\end{array}$} & \multicolumn{4}{|c|}{$\begin{array}{l}\text { Common support after balancing } \\
\text { GPS (weighted t-stats) } n=1380\end{array}$} \\
\hline & Q1 & Q2 & Q3 & Q4 & Q1 & Q2 & Q3 & $\mathrm{Q4}$ & Q1 & Q2 & Q3 & Q4 \\
\hline U.S. population & 21.14 & -1.16 & -3.86 & -14.54 & 2.61 & -0.87 & 0.29 & -1.03 & 1.13 & -0.85 & 0.40 & -0.79 \\
\hline U.S. population ${ }^{2}$ & 20.62 & -1.06 & -3.53 & -14.63 & 2.47 & -0.89 & 0.35 & -1.18 & 1.08 & -0.88 & 0.45 & -0.75 \\
\hline Mex. Population & 9.27 & 5.13 & -2.88 & -11.78 & 2.32 & 1.27 & -0.40 & -2.64 & 1.28 & 1.45 & -0.30 & -2.57 \\
\hline Mex. population ${ }^{2}$ & 9.12 & 5.20 & -2.74 & -11.85 & 2.24 & 1.29 & -0.37 & -2.71 & 1.23 & 1.46 & -0.27 & -2.63 \\
\hline U.S. GSP & 20.79 & -1.21 & -3.43 & -14.71 & 2.39 & -0.90 & 0.30 & -1.08 & 0.90 & -0.88 & 0.42 & -0.85 \\
\hline U.S. GSP ${ }^{2}$ & 19.97 & -1.06 & -2.97 & -14.76 & 2.21 & -0.92 & 0.38 & -1.02 & 0.84 & -0.91 & 0.49 & -0.79 \\
\hline Mex. GSP & 5.36 & 0.88 & -1.11 & -5.14 & 1.48 & 0.42 & 0.22 & -1.36 & 1.16 & 0.51 & 0.25 & -1.38 \\
\hline Mex. GSP ${ }^{2}$ & 5.21 & 0.89 & -1.03 & -5.10 & 1.44 & 0.43 & 0.24 & -1.40 & 1.14 & 0.51 & 0.26 & -1.42 \\
\hline U.S. pop. growth & 6.00 & 2.59 & -2.42 & -6.20 & 1.16 & 1.09 & -0.11 & -1.44 & 1.12 & 1.04 & -0.12 & -1.32 \\
\hline Mex. pop. growth & -2.92 & 2.34 & 0.80 & -0.19 & 0.45 & 0.73 & 0.38 & -0.50 & 0.65 & 0.79 & 0.36 & -0.53 \\
\hline U.S. GSP growth & -6.90 & 0.02 & 3.11 & 3.73 & -0.49 & 1.06 & 1.03 & 0.21 & -0.25 & 0.97 & 0.98 & 0.21 \\
\hline Mex. GSP growth & -1.10 & 1.21 & 1.72 & -1.82 & -0.36 & 0.58 & 0.93 & 0.60 & -0.37 & 0.81 & 0.91 & 0.52 \\
\hline Adjacency & 1.31 & 1.28 & 0.26 & -2.85 & 0.45 & 0.48 & 0.26 & 0.13 & 0.27 & 0.31 & -0.28 & 0.12 \\
\hline Distance & -13.11 & -0.71 & 9.23 & 4.23 & -4.46 & -0.75 & 1.98 & 0.69 & -3.92 & -0.79 & 2.08 & 0.34 \\
\hline U.S. unemployment & 10.08 & -0.28 & -1.65 & -8.05 & 1.47 & 0.04 & 0.67 & -0.38 & 0.88 & 0.05 & 0.69 & -0.28 \\
\hline Mex. unemployment & 1.19 & -1.28 & -3.53 & 3.62 & 1.68 & 0.02 & -0.53 & 0.11 & 1.67 & 0.07 & -0.52 & 0.13 \\
\hline U.S. Gini & 8.55 & -1.58 & -2.07 & -4.79 & 1.31 & -0.41 & 0.45 & -0.52 & 0.59 & -0.40 & 0.53 & -0.36 \\
\hline Mex. Gini & 6.08 & 3.95 & -3.18 & -6.89 & 1.31 & -0.06 & -1.24 & 0.34 & 0.76 & 0.14 & -1.14 & 0.27 \\
\hline Avg. absolute t-stat & \multicolumn{4}{|c|}{$5.35,24 / 72<|1.96|$} & \multicolumn{4}{|c|}{$0.97,63 / 72<|1.96|$} & \multicolumn{4}{|c|}{$0.80,68 / 72<|1.96|$} \\
\hline
\end{tabular}


Table A.12. OLS estimation, dependent variable: $\ln$ (exports)

\begin{tabular}{|c|c|c|}
\hline Covariates and statistics & Estimated coefficient & Standard error \\
\hline Migrant stock & $1.17^{* * *}$ & 0.31 \\
\hline Migrant stock $^{2}$ & $-0.20 * * *$ & 0.07 \\
\hline Migrant stock $^{3}$ & $0.01 * * *$ & 0.00 \\
\hline GPS & $-6.26 * * *$ & 2.61 \\
\hline Migrant stock $\times$ GPS & $1.60^{* * *}$ & 0.46 \\
\hline Constant & $11.70 * * *$ & 0.62 \\
\hline Observations & 1380 & \\
\hline$R^{2}$ & 0.10 & \\
\hline
\end{tabular}


Table A.13. State-state observations excluded by modified common support condition, starting sample of $n=1488$

\begin{tabular}{|c|c|c|c|c|c|}
\hline$\overline{\text { Arizona }}$ & Baja California & South Dakota & Baja California Sur & $\overline{\text { Texas }}$ & Zacatecas \\
\hline Arizona & Sonora & South Dakota & Colima & Vermont & Aguascalientes \\
\hline California & Baja California & South Dakota & Campeche & Vermont & Baja California \\
\hline California & Veracruz & South Dakota & Quintana Roo & Vermont & Baja California Sur \\
\hline Maine & Baja California Sur & Texas & Chihuahua & Vermont & Colima \\
\hline Maine & Colima & Texas & Chiapas & Vermont & Campeche \\
\hline Maine & Quintana Roo & Texas & Coahuila & Vermont & Coahuila \\
\hline Montana & Colima & Texas & Durango & Vermont & Morelos \\
\hline Montana & Campeche & Texas & Guerrero & Vermont & Nayarit \\
\hline Montana & Quintana Roo & Texas & Guanajuato & Vermont & Nuevo Leon \\
\hline North Dakota & Baja California Sur & Texas & Hidalgo & Vermont & Quintana Roo \\
\hline North Dakota & Colima & Texas & Jalisco & Vermont & Queretaro \\
\hline North Dakota & Campeche & Texas & Michoacan & Vermont & Sinaloa \\
\hline North Dakota & Morelos & Texas & Mexico & Vermont & Sonora \\
\hline North Dakota & Quintana Roo & Texas & Nuevo Leon & Vermont & Tlaxcala \\
\hline North Dakota & Yucatan & Texas & Oахаса & Vermont & Tamaulipas \\
\hline New Hampshire & Baja California Sur & Texas & Puebla & Vermont & Yucatan \\
\hline Rhode Island & Baja California Sur & Texas & San Luis Potosi & \multirow{2}{*}{\multicolumn{2}{|c|}{ West Virginia Baja California Sur }} \\
\hline Rhode Island & Colima & Texas & Tamaulipas & \multirow{2}{*}{\multicolumn{2}{|c|}{ West Virginia Quintana Roo }} \\
\hline Rhode Island & Quintana Roo & Texas & Veracruz & & \\
\hline
\end{tabular}


Table A.14. State-state observations excluded by modified common support condition, starting sample of $n=1536$

\begin{tabular}{|c|c|c|c|c|c|}
\hline Arizona & Baja California & South Dakota & Baja California Sur & Texas & Zacatecas \\
\hline California & Baja California & South Dakota & Colima & Vermont & Aguascalientes \\
\hline Maine & Baja California Sur & South Dakota & Campeche & Vermont & Baja California \\
\hline Maine & Colima & South Dakota & Quintana Roo & Vermont & Baja California Sur \\
\hline Maine & Quintana Roo & Texas & Chihuahua & Vermont & Colima \\
\hline Montana & Colima & Texas & Chiapas & Vermont & Campeche \\
\hline Montana & Campeche & Texas & Durango & Vermont & Coahuila \\
\hline Montana & Quintana Roo & Texas & Guerrero & Vermont & Morelos \\
\hline North Dakota & Baja California Sur & Texas & Guanajuato & Vermont & Quintana Roo \\
\hline North Dakota & Colima & Texas & Hidalgo & Vermont & Queretaro \\
\hline North Dakota & Campeche & Texas & Jalisco & Vermont & Sinaloa \\
\hline North Dakota & Morelos & Texas & Michoacan & Vermont & Sonora \\
\hline North Dakota & Quintana Roo & Texas & Mexico & Vermont & Tabasco \\
\hline North Dakota & Yucatan & Texas & Mexico City & Vermont & Tlaxcala \\
\hline New Hampshire & Baja California Sur & Texas & Nuevo Leon & Vermont & Tamaulipas \\
\hline New Hampshire & Baja California Sur & Texas & Оахаса & Vermont & Yucatan \\
\hline New Hampshire & Quintana Roo & Texas & San Luis Potosi & Vermont & Tamaulipas \\
\hline Rhode Island & Baja California Sur & Texas & Tamaulipas & West Virgi & Baja California Sur \\
\hline Rhode Island & Colima & Texas & Veracruz & West Virgi & Quintana Roo \\
\hline Rhode Island & Quintana Roo & & & & \\
\hline
\end{tabular}


Table A.15. State-state observations excluded by modified common support condition, starting sample of $n=1426$

\begin{tabular}{llllll}
\hline Arizona & Baja California & North Dakota & Colima & Vermont & Colima \\
Arizona & Chihuahua & North Dakota & Campeche & Vermont & Campeche \\
Arizona & Sonora & North Dakota & Quintana Roo & Vermont & Coahuila \\
California & Baja California & New Hampshire & Baja California Sur & Vermont & Morelos \\
California & Chihuahua & New Hampshire & Quintana Roo & Vermont & Nuevo Leon \\
California & Mexico & Rhode Island & Baja California Sur & Vermont & Quintana Roo \\
California & Oaxaca & Rhode Island & Colima & Vermont & Queretaro \\
California & Puebla & Rhode Island & Quintana Roo & Vermont & Sinaloa \\
California & Veracruz & South Dakota & Baja California Sur & Vermont & Sonora \\
Maine & Baja California Sur & South Dakota & Campeche & Vermont & Tabasco \\
Maine & Colima & South Dakota & Colima & Vermont & Tlaxcala \\
Maine & Quintana Roo & South Dakota & Quintana Roo & Vermont & Tamaulipas \\
Montana & Colima & Vermont & Aguascalientes & Vermont & Yucatan \\
Montana & Campeche & Vermont & Baja California & West Virginia Baja California Sur \\
Montana & Quintana Roo & Vermont & Baja California Sur & West Virginia Quintana Roo \\
North Dakota & Baja California Sur & & & &
\end{tabular}


Figure 2.1. Percentage distribution of matrículas consulares vs. U.S. Census

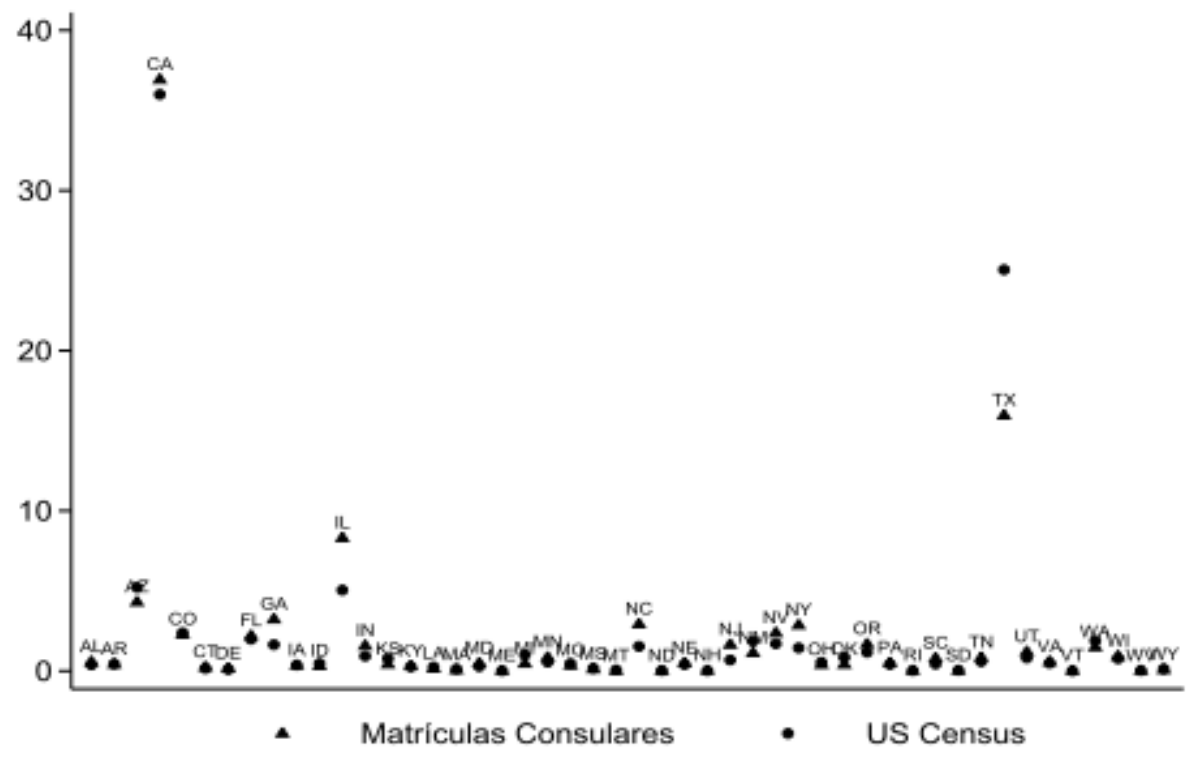

Figure 2.2. Mexican states average education and migration

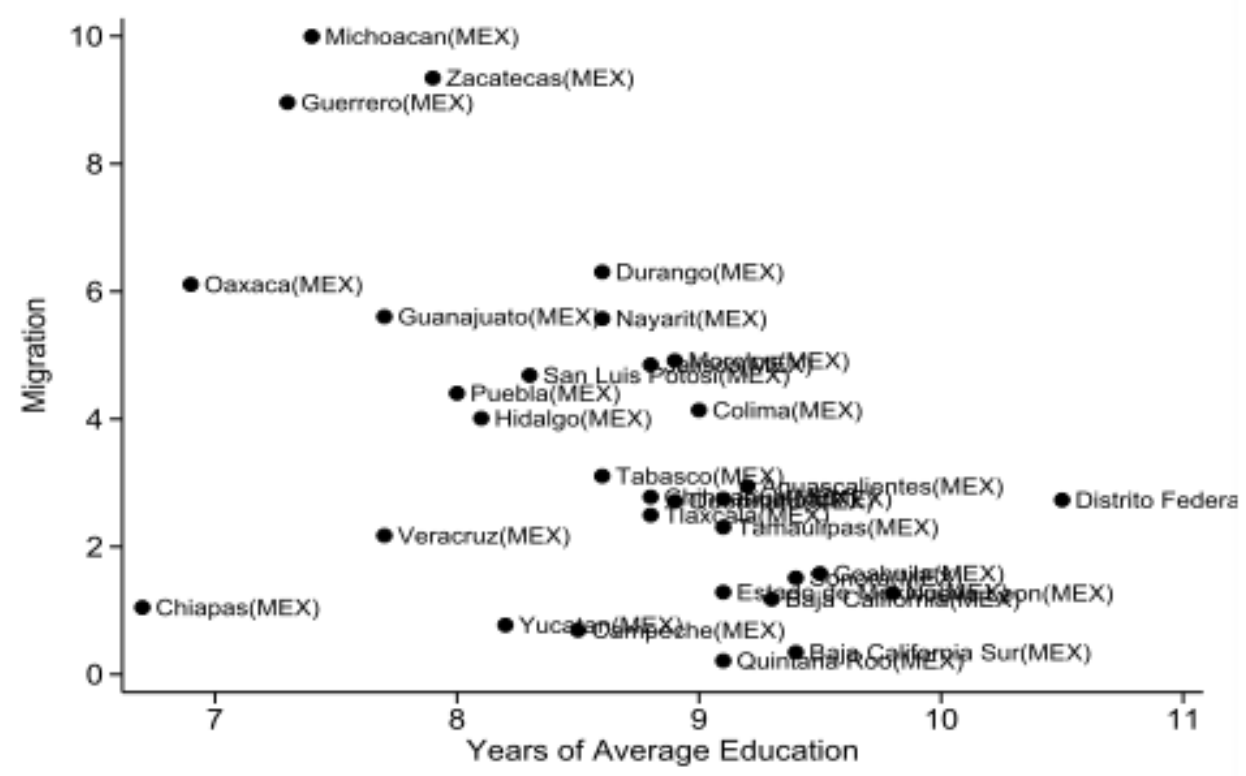


Figure 2.3. Dose response function and treatment effect function, $n=1429$
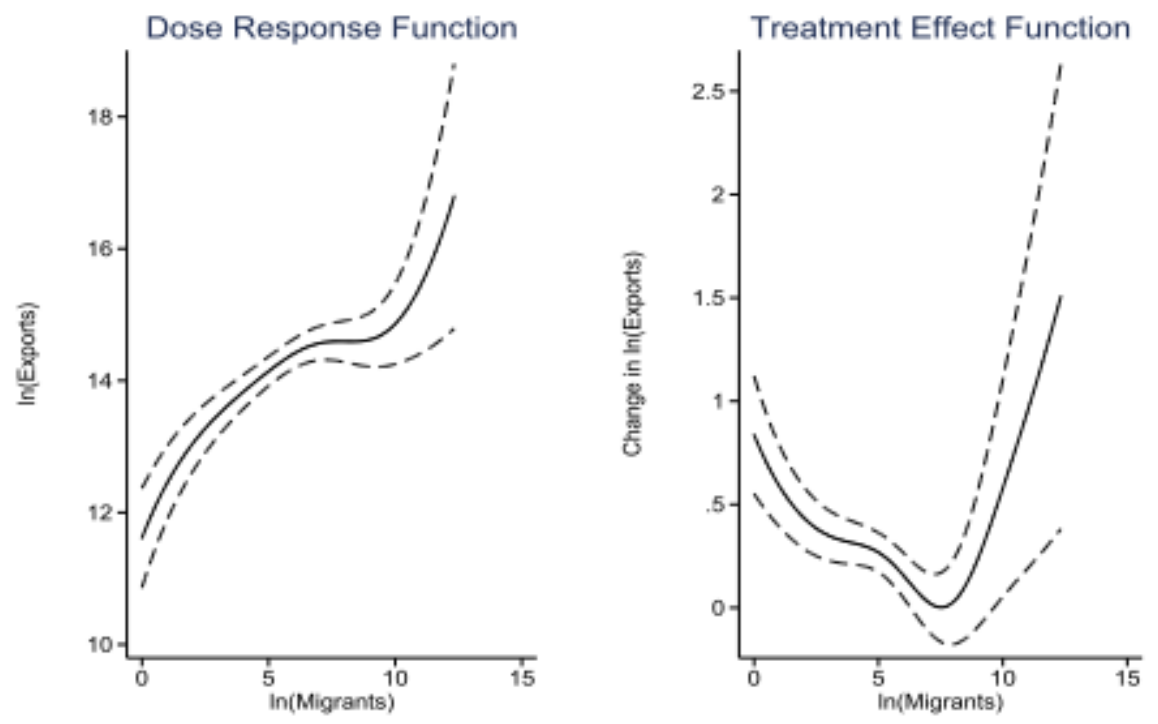

Notes: 90 percent confidence intervals, represented by dashed lines, are constructed by bootstrapping.

Figure 2.4. Marginal contributions for migrants $>500$

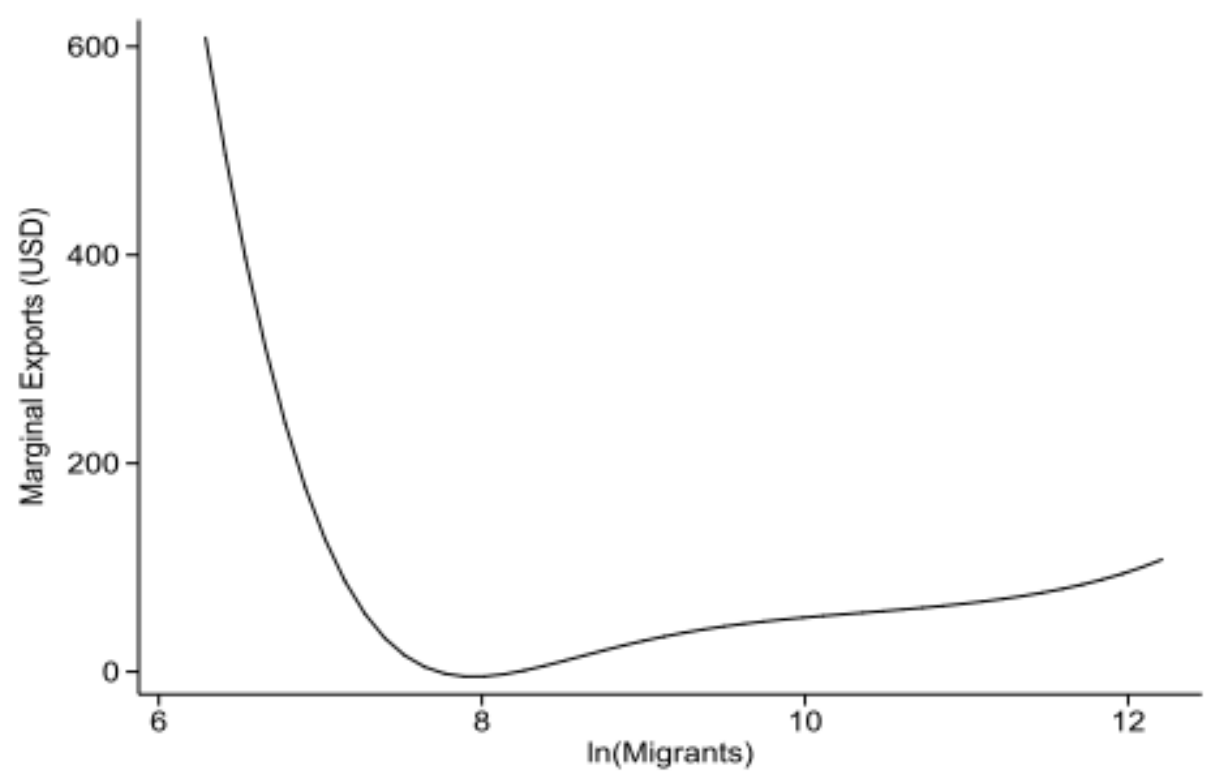


Figure 2.5. Marginal contributions for migrants $<\mathbf{5 0 0}$

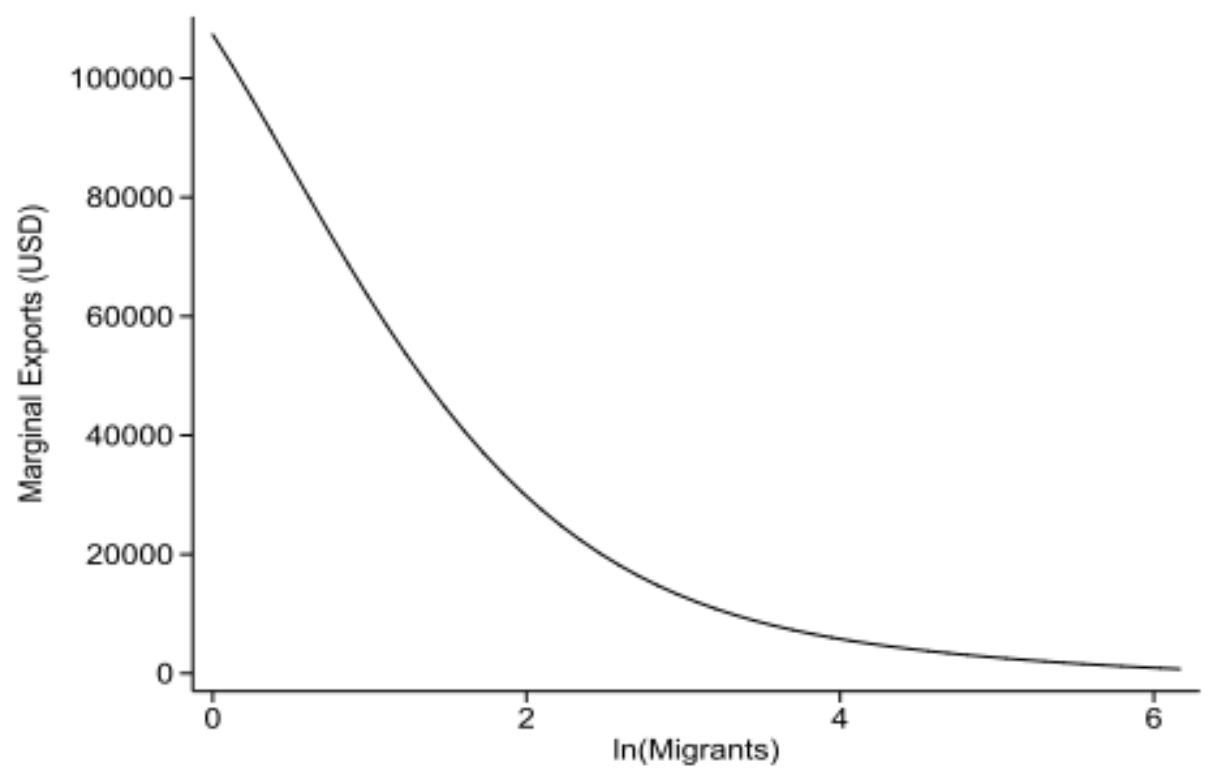

Figure A.1. Migration and trade, simple correlation

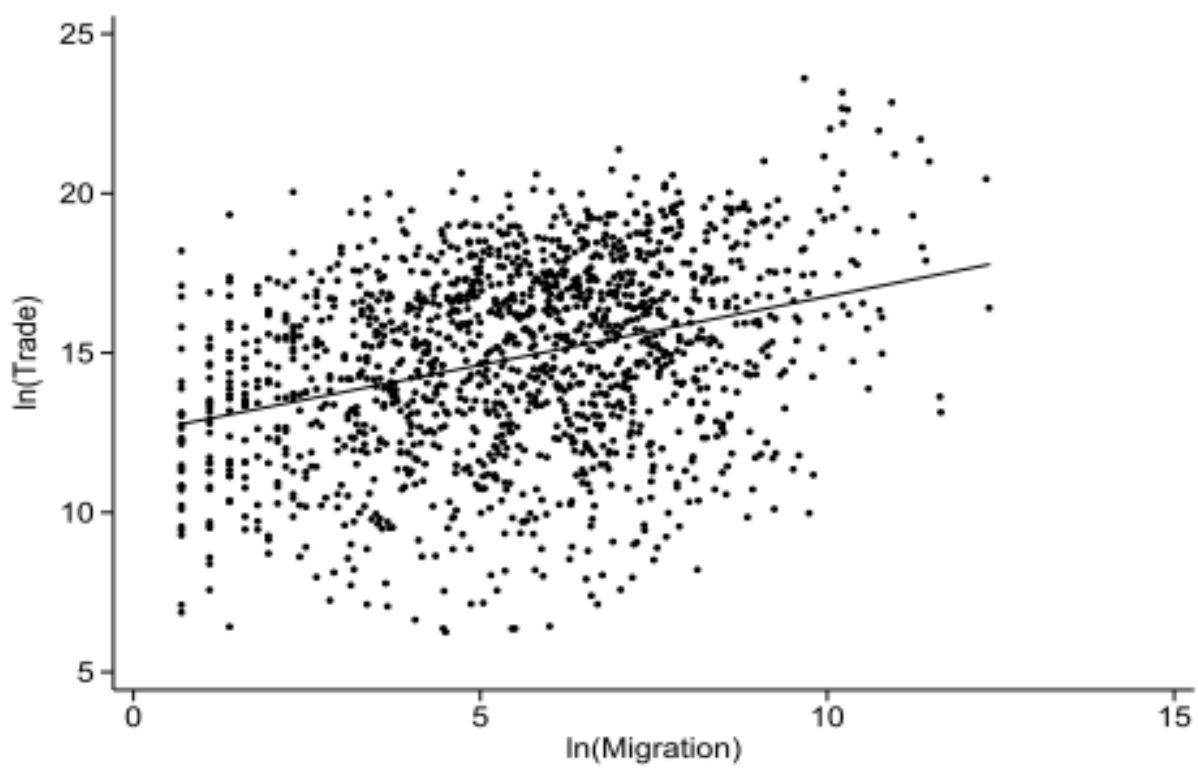

Notes: Migration is measured as the logarithm plus one of matrículas consulares from 2006-2010, while trade is measured as the logarithm plus one of trade in 2010; only values greater than zero are included. 
Figure A.2. Dose response function and treatment effect function, $n=1478$
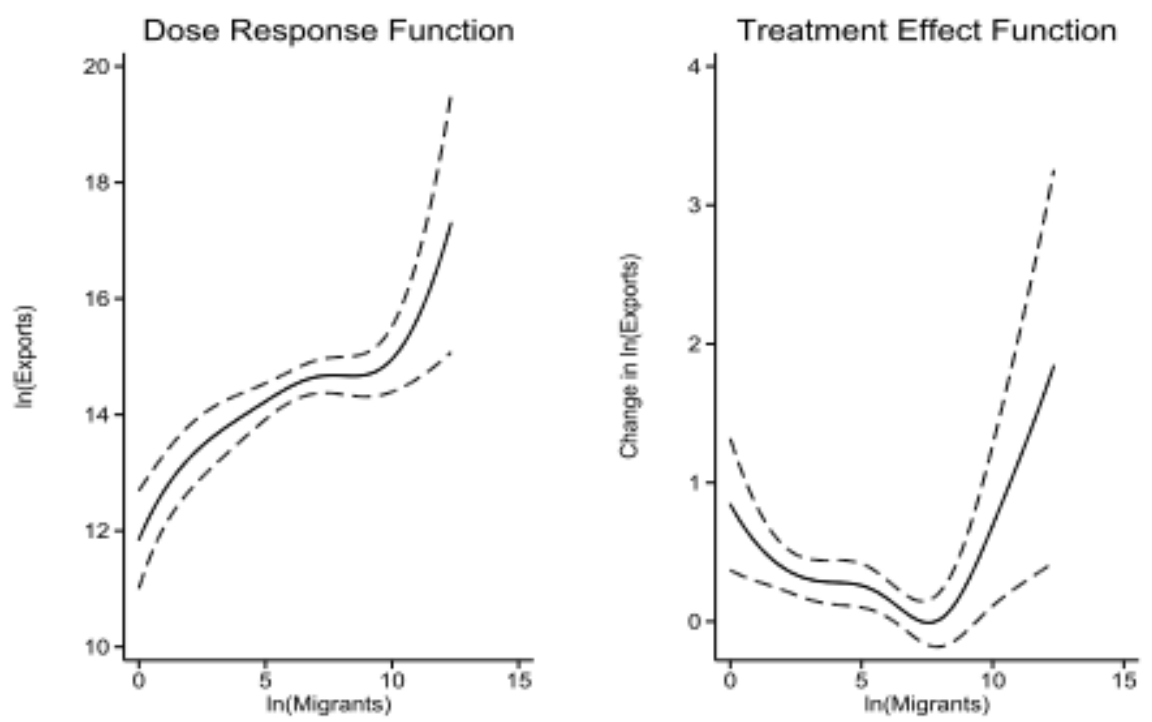

Figure A.3. Dose response function and treatment effect function, $\boldsymbol{n}=\mathbf{1 3 8 0}$
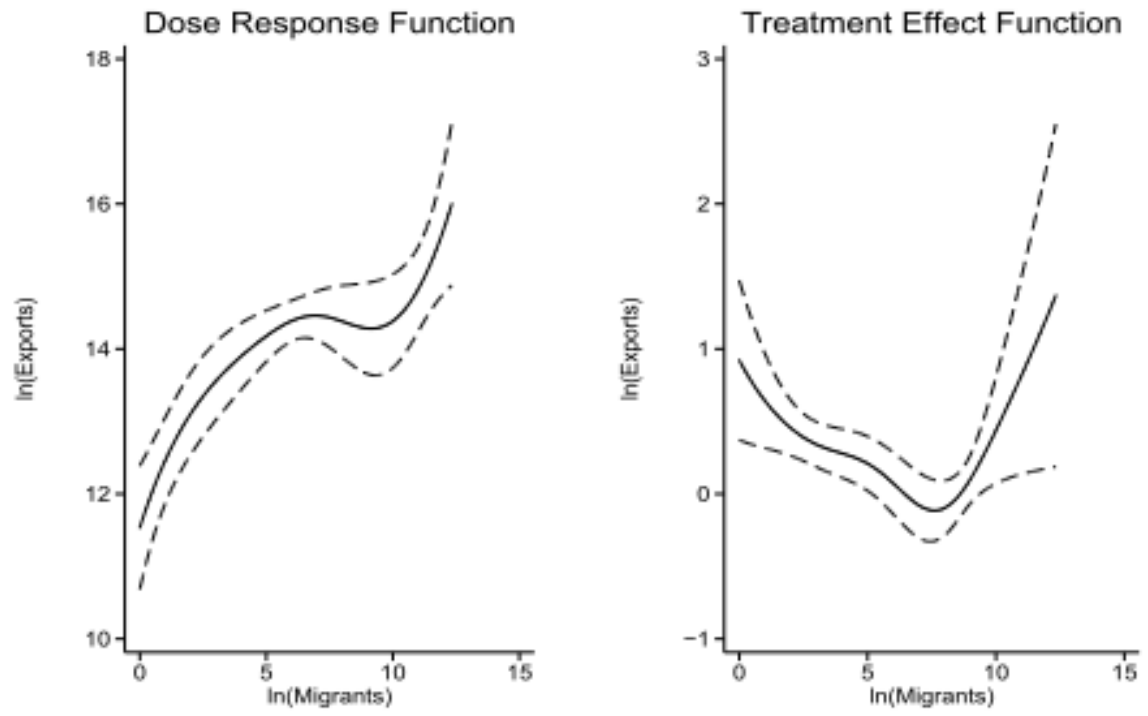


\section{CHAPTER III}

\section{WAGE GAPS, MIGRATION, AND DEVELOPMENT:}

\section{A VIEW FROM BOTH SIDES OF THE BORDER}

\section{Introduction}

If a Mexican-born U.S. resident filed a visa application for a sibling living in Mexico 16 years, 11 months ago, that resident and sibling would just now be receiving a verdict. If the U.S. resident happened to be born in the Philippines, having filed an application for a sibling still living in the Philippines, they would be waiting even longer currently up to 23 years and 7 months. ${ }^{47}$ While these astonishingly long lines at the United States border are clearly partly driven by U.S. immigration policy restrictions, specifically yearly quotas limiting the supply of immigrant visas depending on visa class and country of origin, the lines also exhibit the demand for entry into the United States. In the above cases, demand is most likely enhanced by the existence of family connections, however the difference in wages and standards of living across countries is arguably the most important factor attracting immigrants from all over the world. This demand is reflected by the fact that 272 potential migrants applied for each visa awarded by the 2010 U.S. Diversity Visa Lottery for entry at-large, the lottery open to those meeting minimum qualifications and not applying through family connections to U.S. residents. This number rose to 393 per available visa in $2012 .^{48}$

\footnotetext{
${ }^{47}$ These scenarios are based on online priority date information from the U.S. Bureau of Consular Affairs. As of September 1, 2013, the website lists a priority date for processing of October 8, 1996 for Mexican siblings, while that of February 15, 1990 for Filipino siblings.

${ }^{48}$ The U.S. Bureau of Consular Affairs' website lists detailed statistics for the Diversity Visa Lottery from 2007-2012.
} 
International migration demands attention from economists on a number of issues, many of which have already been examined extensively by the literature. Among a wide variety of topics, researchers focus on effects of immigration on the destination country (Amuedo-Dorantes and de la Rica, 2007); effects of emigration on the origin country (Taylor and Dyer, 2009); remittances (Stark, 2009); migrant selection (McKenzie et al., 2010); and most recently, factors underlying individual attitudes toward immigration (Card et al., 2012). The recognition of the extreme amount of demand for entry in excess of permitted supply raises the issue of efficiency gains from the relaxation of existing restrictions on immigration, in addition to the separate need for increased attention to the effects of migration on the migrants themselves. Only a handful of researchers have started to tackle these themes, especially crucial to potential migrants outside the OECD countries, given that studies generally find huge economic gains accruing to those who actually cross the border into the wealthier countries. For example, Clemens et al. (2008), hereafter CMP, calculates wage ratios for 42 developing countries of observably equivalent workers residing in the US compared to residing in the respective country of origin, adjusting for selection, estimating a median of over 4 for all countries examined. Furthermore, of the three areas of globalization (goods, capital, and labor), efficiency arguments clearly point to restrictions on labor mobility as the single largest remaining cause of market distortions and efficiency losses in the world economy, with goods and capital already enjoying much higher levels of relatively unrestrained mobility. Pritchett (2006) succinctly signals the huge disparity in possible worldwide efficiency gains from the three areas of globalization, comparing a gain of $\$ 65$ billion from complete capital liberalization (Caselli and Feyrer, 2007), a gain of $\$ 107$ billion from complete trade liberalization (World Bank, 2005), and 
the massive gain of $\$ 65$ trillion from complete labor mobility (Hamilton and Whalley, 1984; Klein and Ventura, 2004). Even a slight relaxation of labor mobility restrictions would result in huge efficiency gains, estimated at $\$ 170$ billion given a 3\% increase in migrants in the labor forces of OECD countries (Walmsley and Winters, 2005).

Accepting the potential gains as a given, the issue turns to one of ethics, as limiting labor mobility is the only remaining widely accepted discrimination based on a characteristic determined by birth, that of national origin. By sustaining strong restrictions on labor mobility, wealthy countries are implicitly valuing potentially minuscule losses to small groups of existing residents more than the huge potential gains to new residents simply based on country of birth, difficult to justify on any ethical grounds. ${ }^{49}$ Both economic efficiency and ethical arguments lead to unequivocal support in favor of more labor mobility, arguments that become even stronger if viewing human mobility in the light of its current and potential contributions to economic development. As stated by Clemens and Pritchett (2008), “...crossing international borders is not an alternative to economic development, it is economic development.” Indeed, 23 of every 100 Mexicans earning more than $\$ 10 /$ day live abroad; 87 of every 100 Haitians earning more than $\$ 10 /$ day live abroad. Simply put, while migration is much more a complement to rather than a substitute for more traditionally accepted development strategies, migration indeed makes a large contribution to the economic development of migrants themselves.

My analysis examines the connection between existing wage gaps, migration, and migration's power for development, coinciding with CMP in its attempt to bring these

\footnotetext{
${ }^{49}$ The total welfare gain or loss related to immigration remains relatively controversial, with recent research from di Giovanni et al. (2013) finding that natives in migrant-receiving countries actually experience long-run gains from increased migration.
} 
issues to the forefront of the international migration and economic development research agendas. My empirical analysis relies heavily on two datasets - first, a novel dataset compiled from personal interviews with migrants in the U.S. as well as households in highmigration communities in Mexico, and second, La Encuesta sobre Migración en la Frontera Norte de México (EMIF) dataset captures human flows between the United States and Mexico. By way of the detailed survey information from both EMIF and my original data, as well as Mexican census data, I allow for a comparison across the differing samples in attempting to answer the following questions: (1) What are the wage gains for migrants arriving to and working in the United States? (2) Where in the origin wage distribution do migrants come from? (3) Where in the origin wage distribution do migrants end up?

In examining the first question, I contribute to the limited number of existing estimates detailing international wage gaps. Mexican household survey information, along with the data mentioned above, allows for the calculation of exact wage gains for the subsample of migrants reporting before- and after-migration wages in both EMIF and the new data. In answering the second, I focus on the issue of selection, clearly relevant in thinking about possible future gains from further migration. I again combine the existing household survey data with the EMIF and new data in pinpointing migrants' starting positions within the origin wage distributions. Finally, in examining the third, I contribute evidence to migration's power of development. Specifically, I take the gains to migrants from question one and place these post-migration wages in the origin wage distribution, allowing for a dynamic comparison of migrants' before- and after-migration wages relative to the relevant origin community. Opting to adopt the approach of Clemens and Pritchett (2008) and focus on people rather than places in terms of development, addressing these 
three questions systematically allows me to obtain succinct measures of migrants' economic development, in addition to the development relative to those remaining in origin communities. In particular, the figures for migrants' wage gains from the subsamples make a much needed contribution; estimates for gains based on comparing outcomes for observably similar workers are somewhat more common, however gains calculated based on comparing outcomes for observably and unobservably identical workers - the same individuals - are few and far between.

I find that the migrant groups under examination enjoy immediate average wage gains from migration of over \$5 (at Purchasing Power Parity) per hour worked, resulting in average income increases of over fivefold. These absolute wage gains translate into average relative movements within origin wage distributions of upwards of 50 and 60 percentiles, respectively, for the main two groups studied. Additionally, all but two of the approximately $40 \%$ of migrants in poverty before migration are able to rise above the poverty line by moving across borders. In continuation, I highlight issues related to the data, discuss the results, and briefly conclude.

\section{Data and Background}

My analysis draws on three datasets: the 2010 Mexican Census, EMIF covering the years 1999-2009, and the original data collected through household interviews in communities both in Mexico (HIMEX) and the United States (HIUS). The EMIF data provides a first look into the actual wage gains accruing to Mexican migrants choosing to cross the border, as the population working in the U.S. and reentering Mexico in order to visit can be correctly identified through the survey results. The population that provides an answer as to wages in the U.S. and the wages earned in Mexico immediately before 
migration form my EMIF sample under examination. It is important to point out that this sample does not address the wage gains question as exactly as we would like, as migrants report current wages in the U.S. as opposed to the wages received immediately after migration to the U.S. during the interview upon reentry into Mexico. Assuming that with time comes added experience, skills, and possible adaptation to a new labor market that contributes to higher productivity and results in higher wages, this clearly has the potential of biasing the apparent wage gains upward, entirely dependent on how much time has passed between time of migration and the time of the EMIF interview. ${ }^{50}$

The novel HIMEX and HIUS data address this shortcoming directly, detailing migrants' wages both immediately before and after migration. Although in some cases the physical process of migration can be a long one, possibly even several months, it is difficult to argue that during that time any new experience, skills, or any other factor that would potentially affect the wage-earning ability of an individual are acquired; this time is spent just migrating. Therefore, the only change affecting wages is physical geographic location; a migrant simply leaves one labor market in order to enter another, allowing for complete identification of the wage gains stemming from migration.

HIMEX and HIUS data are the result of household interviews, conducted both in Mexico and the U.S. during 2012, following a format similar to that of the Mexican Migration Project (MMP). HIMEX comprises 264 interviews in the state of Veracruz, Mexico, a state for which U.S.-destined migration has increased in recent years, while

\footnotetext{
${ }^{50}$ Length of time between migration and the EMIF interview varies across the waves of the EMIF, clearly increasing in recent years, with the averages as follows: 33 months in 2009, 25 months in 2008, 24 months in 2007, 19 months in 2006, 18 months in 2005, 17 months in 2004, 16 months in 2003, 13 months in 2002, 13 months in 2001, 11 months in 2000, and 11 months in 1999.
} 
HIUS is formed by 187 interviews in South Florida. While the HIMEX and HIUS samples do not necessarily provide data representative of the entire Mexico-U.S. migrant population, they do contribute two snapshots that are representative at the respective community level, as households are randomly selected within communities for participation. Random selection is based on a process of dividing communities into equally-sized blocks based on satellite map imagery, and the subsequent verification of mapping of all community blocks in person upon arrival at each specific location. ${ }^{51}$ The random selection on both sides of the border provides a key difference between my data and that of the MMP. The MMP uses the "snowball method" relying on personal references in U.S. migrant communities; since random selection is used in both Veracruz and South Florida in my survey, both samples indeed are representative of the communities surveyed. In addition to absolute wage gains, relative income gains are often argued to be a potential benefit of importance to migrants. If an individual's income is not only increasing in absolute terms, but is also growing relative to that of the appropriate reference population, migration may be even more attractive. ${ }^{52}$ The Census data allows for the construction of the origin wage distributions from which the migrant sample migrates. ${ }^{53}$ In turn, inserting the HIMEX and HIUS pre- and post-migration wages into the respective

\footnotetext{
${ }^{51}$ HIMEX and HIUS refusal to participate rates reach $12 \%$ and $25 \%$, respectively. The number of observations containing both pre- and post-migration wages included in the calculation of the results presented in the remainder of the paper are 128 and 69, respectively, due to incomplete answering of the survey.

${ }^{52}$ See Stark and Taylor (1991) for an exposition on the potential importance of these relative differences, coined relative deprivation.

${ }^{53}$ The 2010 Census includes data on wages and various units of time of the wages reported, allowing for a simple calculation of the hourly wage.
} 
origin wage distributions permits a clear comparison of the relative position of migrants in the associated income distributions pre- and post-migration.

For purposes of comparison, all wages are converted to 2011 US dollars at Purchasing Power Parity (P\$) by using World Bank (2012) GDP conversion factors, as well as the US CPI-U series. Observations containing apparent reporting errors as to wages are dropped from the EMIF and Census data. ${ }^{54}$ I use eleven waves of EMIF data, the average sample size being 6,480. Those migrants reporting wages earned in the U.S. and wages earned in Mexico immediately before migration average 480 per wave. I extract the Census data for the nine states and fifteen different Mexican municipios (municipalities) represented in the HIMEX and HIUS populations, then examining the relative gains at the municipality level. Approximately one-third of Census observations contain wage information, examined municipalities ranging from 1,323 to 17,721 in the number of reported wages. ${ }^{55}$

Table 3.1 outlines summary statistics from the gathered data on migrants, including mean, median, standard deviation, and range for each of the two main samples. In comparing the two samples, on average HIMEX migrants have higher pre- and postmigration wages; furthermore, they are slightly younger at the time of the interview, more likely to be male, more educated, and less settled in destination communities (by number of years since migration), relative to HIUS migrants. Additionally, $66 \%$ and $80 \%$ of HIMEX and HIUS migrants are married, respectively. HIMEX migrants are more likely to

\footnotetext{
${ }^{54}$ This includes hourly wages less than $\$ 0.20$ (2011 P\$) and clear reporting errors, such as an hourly wage for manual labor of $\$ 450$ or $\$ 1050$.

${ }^{55}$ Due to lack of available data, Mexico City, which encompasses various municipalities, is not examined at the municipality level.
} 
send remittances than HIUS migrants (21\% and 25\%, respectively, report sending no remittances); those HIMEX migrants who do send remittances send more on average than HIUS migrants, the respective averages representing $18 \%$ and $12 \%$ of post-migration wages. ${ }^{56}$

\section{Results and Discussion}

Absolute wage gains

In discussing the results, I use the following straightforward definitions for wage differences $\left(D_{W i}\right)$ and wage ratios $\left(R_{W i}\right):\left(D_{W i}\right)=\left(W_{i, p o s t}-W_{i, p r e}\right)$, where $W_{i, p o s t}$ and $W_{i, p r e}$ are hourly wages for individual $i$ adjusted to 2011 P\$ immediately post- and premigration, respectively, and $R_{W i}=\frac{W_{i, p o s t}}{W_{i, p r e}}$.

\section{HIMEX}

Figure 3.1 summarizes the pre- and post-migration wages for HIMEX emigrants. On average, a HIMEX emigrant gains P\$7.06 in hourly wages by leaving Mexico and entering the U.S. This gain in wages is immediate, representing the wage gains due purely to geographic relocation. Table 3.2 and Figure 3.2 signal that this average disguises considerable variation in gains across the population, with $D_{W i} \in[3.44,20.53]$, however all emigrants certainly enjoy positive wage gains from crossing the border. Considering an alternative measure of wage gains, that of wage ratio $R_{W i}$, the average ratio of HIMEX emigrants is 5.24, meaning that emigrants face earning more than five times in postmigration wages than those earned immediately before migration. Figure 3.3 highlights the

\footnotetext{
56 This percentage calculation assumes a 40-hour workweek and four weeks of monthly work, as remittances are reported by migrants on a monthly basis.
} 
variation across individuals in $R_{W i}$, this measure ranging from 1.91 to 13.65 . Without a benchmark for comparison, it is difficult to judge whether these gains are "small" or “large,” although at first glance the gains to migrants seem quite substantial. However, simple observation using measures of poverty helps to start put in perspective the magnitude of gains accruing to migrants willing and wanting to cross the border in the HIMEX sample. A considerable amount of the HIMEX population is moving out of poverty, whether using the Mexican or U.S. definition of the poverty line. Immediately before undertaking migration, 30\% of HIMEX migrants are below the Mexican poverty line of \$1904 (unadjusted, per individual) pesos per month established by the Consejo Nacional de Evaluación de la Política de Desarrollo Social (CONEVAL). ${ }^{57}$ Immediately after migration, with an average wage of P\$8.92 per hour, zero HIMEX migrants are in poverty measured by the 2012 U.S. standard of $\$ 11,170$ (unadjusted, per individual) annually; the entire portion of impoverished individuals not only springboards out of poverty by the Mexican measure, poverty is no longer found in this population even when measured by the more stringent U.S. poverty line.

\section{HIUS}

Figure 3.4 shows hourly wages for the HIUS migrants pre- and post-migration. By visual inspection, the distributions are quite similar to those of the HIMEX sample; Table 3.2 highlights that HIUS migrants have a slightly lower pre- and post-migration hourly wage than their HIMEX counterparts, averaging P\$1.75 and P\$6.96, respectively. Average absolute wage gains of $\mathrm{P} \$ 5.22$ accrue to HIUS migrants, coming up short of those of

\footnotetext{
${ }^{57}$ For ease of exposition, I use the most recent poverty line pobreza patrimonial from 2008, although the CONEVAL has also adopted a multidimensional strategy for measuring poverty in Mexico based on factors other than just income.
} 
HIMEX migrants. However, the distribution of those gains exhibits more homogeneity for HIUS migrants than for HIMEX migrants, with $D_{W i} \in[1.77,8.80]$ as evidenced in Figure 3.5 .

As displayed in Figure 3.6, HIUS migrants enjoy an average wage ratio of 5.56, nearly mirroring the HIMEX average wage ratio; even the migrant gaining the least in magnitude still enjoys considerable wage gains, enjoying a wage ratio of 1.41. Again framing the evidence in terms of the Mexican and U.S. poverty lines, $63 \%$ of HIUS migrants live in poverty pre-migration, earning less than \$1904 pesos per month. Astoundingly, the act of migrating moves all but two migrants above the U.S. poverty line of $\$ 11,170$ annual dollars in post-migration wages, while zero are left in poverty by the Mexican measure.

EMIF

Although the variation in time elapsed between each individual's migration and the corresponding EMIF survey is problematic for identifying the wage gains due solely to geographic mobility, the data provides a benchmark for comparing the gains found in the HIMEX and HIUS samples, especially useful since the EMIF provides a representative sample of Mexican border crossers. ${ }^{58}$ Figure 3.7 depicts the absolute wage gains accruing to EMIF-surveyed migrants, showing the distribution of pre-migration versus postmigration hourly wages. For both wage distributions and the resulting wage ratios displayed in Figure 3.8, results are extremely consistent across the eleven EMIF waves examined, with only the slight amount of variation noted by comparing averages (medians)

\footnotetext{
${ }^{58}$ See Secretaría del Trabajo y Previsión Social (2012) for details on methodology of the EMIF.
} 
from Table 3.2. The full EMIF samples exhibit a wage difference range (in averages) from a minimum of $\mathrm{P} \$ 6.20$ (2003) to a maximum of $\mathrm{P} \$ 7.68$ (2009); wage ratio averages lie between 4.65 (2003) and 5.29 (1999 and 2009). While HIUS average wage differences fall short of those of the EMIF samples due to overall lower wages (both before and after migration), both HIMEX and HIUS wage ratios reside near the upper limit of the EMIF samples, HIUS average wage ratios eclipsing all EMIF averages at 5.56 .

In the interest of further detailing the true amount of wage gains attributable solely to migration, I trim the EMIF samples by analyzing wage changes for only those migrants who report fewer than twelve months elapsed between migration and the EMIF interview. With this limited window of time, it is reasonable to assume that the U.S. wages reported in the EMIF interview are equal to the wages received immediately after migration. Given the expectation that both wage ratios and wage differences are biased upward as longer time periods elapse between migration and the EMIF survey, the revised samples should return both attenuated wage differences and wage ratios compared to those of the full EMIF samples. Table 3.2 confirms the hypothesized effect, as both wage differences and wage ratios drop across all EMIF waves for the subsample reporting less than twelve months elapsed. However, perhaps somewhat surprisingly the decreases are not large in magnitude, leaving two possible explanations. First, migrant workers may not be enjoying as much wage growth as expected after arrival in the U.S., most wage gains actually accruing immediately upon arrival due exclusively to migration. Second, differences in time elapsed between the trimmed sample of less than twelve months and the rest of the sample may not be large enough to permit wage growth over time to take on any magnitude of significance in the present analysis. Average wage differences ranging from $\mathrm{P} \$ 5.87$ (2003) to $\mathrm{P} \$ 7.14$ 
(2009) approach those of HIMEX and HIUS migrants, while HIMEX and HIUS average wage ratios lie just above the trimmed samples' range of 3.92 (2008) to 5.22 (1999).

In further examining these results, I again rely on CMP and Pritchett (2006) in making comparisons with various wage ratios discussed in the literature. CMP provides a convenient benchmark estimate for comparison with $R_{W i}$, however it is important to note that this estimate is generated from much different data and observably equivalent workers, adjusted for selection, while my figures are calculated comparing observably and unobservably equivalent workers; the CMP measure of $R_{e}$ (comparable to $R_{W i}$ ) for Mexico is estimated as 2.46, after adjustment for selection. Pritchett (2006) highlights other types of wage discrimination dependent on aspects such as race or gender; for example, the 1995 male-female wage ratio in the US is estimated as 1.3 and in the median country as 1.4 , while the 1939 US white-black wage ratio has been estimated at 1.6. While these wage ratios pale in comparison to both the $R_{e}$ of CMP and even the lowest of my calculated wage ratios, ironically they receive much more attention than the wage ratios mentioned in this paper. With no formal restrictions on worker mobility, one might imagine a $R_{W i}$ approaching the geographic ratios also mentioned in Pritchett (2006) of the 1999 urbanrural wage ratio of 1.4 averaged over 43 countries, or the 1999 US-Puerto Rico wage ratio of $1.5 .^{59}$

Relative wage gains

\footnotetext{
${ }^{59}$ Even with no restrictions on worker mobility, it is reasonable to expect the sustainability of wage ratios greater than 1, as the costs of moving, including potential differences in language, customs, culture, etc. make relocating less attractive relative to the origin.
} 
Examining relative wage gains provides a second perspective on the economic benefits migrants enjoy by moving across the border. The widely-studied idea of relative deprivation highlighted in Stark and Taylor (1991) forms the theoretical foundation for my strategy of placing the migrant pre- and post-migration wages into the wage distribution of the origin communities, specifically at the municipality level. This allows for a straightforward inspection of where in the distribution migrants come from, and where in the distribution migrants end up. The selection of the origin community as the relevant reference group is simple to justify as the majority of migrants in the HIMEX and HIUS samples maintain strong connections with family members in Mexico, migrants often crossing the border without their immediate families. Anecdotal evidence confirms this idea as well, as several interviewees in Mexico explained, "If you want to talk to the migrant families, just look for the nicer houses.” Some of these houses are the result of individual male migrants sending money to their respective wives who have stayed behind in Mexico, while some are the result of accumulated savings for a migrant who decided to make the move back to Mexico after some time in the U.S. - in either case, the evidence indeed points to the origin community as the appropriate group of reference. While reference groups may be subject to partial or complete revision if a migrant becomes more (less) connected to the destination (origin) community, I assume this possibility away, especially given that the measured wage gains accrue immediately upon migration, when migrants are arguably just as connected to origin communities as before undertaking migration. ${ }^{60}$

\footnotetext{
${ }^{60}$ I relax this assumption in the following subsection, examining the possibility of revised groups of reference.
} 
HIMEX

Figure 9 clearly shows the movement in the origin wage distribution that HIMEX migrants make. Pre-migration wages are concentrated in the lower half of the Census wage distribution, with the dashed-line bars representing these wages before relocation. Postmigration wages move to the right tail of the distribution, dotted bars representing these relatively high wages after relocation. Figure 3.10 paints an even clearer picture of the relative movement, as it displays the percentiles of the origin wage distribution from which migrants come and the percentiles in which they end up immediately after migrating. HIMEX migrants are fairly evenly spread out among the Census distribution before migration, although the majority clearly comes from below the median wage. However, the dotted bars represent the dramatic post-migration change; all migrants move to the upper two deciles of the origin wage distribution simply by migrating. Migrant premigration wages are spread between the 3rd and 92nd percentiles of the Census wage distribution, the average coming from the 38th and the median from the 40th; postmigration wages all fall in the upper section of Census wages between the 84th and 99th percentiles, the average falling in the 90th and the median in the 91st. As a result, the average HIMEX migrant leapfrogs over 52 percent of the reference population by undertaking migration.

HIUS

HIUS migrants come from an assortment of origin wage distributions, spread across 14 municipalities in 8 different Mexican states. Figure 3.11 summarizes the relative gains 
for all HIUS migrants across all represented municipalities, although migrants are placed in the respective origin wage distributions separately in order to arrive at the displayed percentiles. HIUS migrants come disproportionately from the lower deciles of the various Census wage distributions, starting from relatively disadvantaged income positions even more than HIMEX migrants. However, movements within the origin wage distributions are quite similar to those of the HIMEX counterparts; HIUS migrants move to the upper deciles of the Census wage distributions, with slightly greater variation in percentile outcomes than HIMEX migrants. HIUS migrant pre-migration wages are spread between the 1st and 84th percentiles of the respective Census wage distributions, the average coming from the 15th and the median from the 9th; post-migration wages fall between the 52nd and 94th percentiles of Census wages, the average falling in the 79th and the median in the 80th. These dramatic changes result in the average HIUS migrant jumping over 64 percent of the population within the origin wage distribution upon migrating.

\section{Are migrants better off after migration?}

Clearly migrants are benefiting from large wage gains; however, income gains do not necessarily translate into a higher level of overall well-being. Costs of migration, both financial and any costs related to a preference for places of origin, cut into the income gains that migrants enjoy, but migrants may also change the relevant group of reference upon migrating or over time as adaptation to the place of destination occurs, this possibility examined recently in Stillman et al. (2012). If the reference group changes, even a higher level of objective well-being (income gains net of any migration costs) could result in a lower level of subjective well-being if migrants now compare their respective situations to the incomes of the destination community that are generally higher than those of the 
community of origin. While revealed preference suggests that migrants are better off after migration, Bartram (2011) argues that migration results in seemingly paradoxical lower levels of well-being. However, Stillman et al. (2012) challenges this conclusion, emphasizing clear higher levels of objective well-being yet complex and conflicting results regarding subjective well-being.

Unfortunately, given the nature of the potential costs of migration, measuring the wage gains to migration is an easier task than measuring the costs. It is nearly impossible to quantify any cost related to differences in language and culture or a preference for place of origin; however, my survey data provides a glimpse as to the large portion of the migration cost related to the actual trip. The actual cost of the migration trip itself averages P\$2249 for HIMEX migrants and P\$2570 for HIUS migrants. ${ }^{61}$ This cost includes and is often dominated by the frequent cost of the hired coyote, responsible for facilitating the border crossing of undocumented migrants. ${ }^{62}$ This up-front cost to migration is certainly large, often requiring borrowing to finance the expense, especially given the origin income levels of the migrants entering the samples. The substantial average costs are equivalent to between 9 and 11 months income for an individual hovering at the Mexican poverty line of $\$ 1904$ pesos per month. However, Table 3.3 provides a contrasting perspective, arguably signaling that this cost is not as large as first appears. Given cost of migration $c_{i}$ and wage difference $D_{W i}$, I calculate the number of hours of work required for each individual migrant to "break even," $E_{i}=\frac{c_{i}}{D_{W i}}$.

\footnotetext{
${ }^{61}$ In all reported statistics related to cost and the subsequent break-even hours, I do not include observations for migrants reporting a total cost of $\$ 0$.

${ }^{62}$ While interviewees are not explicitly asked about legal status or the use of coyotes, many volunteer information, detailing both the cost of the coyote as well as the total cost of migration.
} 
Assuming a 40-hour workweek, the average HIMEX migrant breaks even near the beginning of the ninth week of work, while the average HIUS migrant must wait until the thirteenth week. In turn, objective well-being is clearly increased through migration, unless those uncaptured recurring costs of migration related to preference for place of origin surpass the remaining wage gains during the first year of migration and also year after year if the migrant prolongs residence in the destination community. While impossible to rule out given lack of data on the other costs of migration, the possibility of a net loss does not appear to be a likely occurrence, as this would require those other costs of migration to average a minimum of $\mathrm{P} \$ 8737$ and $\mathrm{P} \$ 5576$ for HIMEX and HIUS migrants per year, respectively, given the average break-even hours and wage gains for each group. ${ }^{63}$

With apparent gains in objective well-being in hand, the last question of my household survey allows for the examination of the subjective well-being of migrants, as well as insight on the relative importance of absolute versus relative gains. "Knowing what you do today, would you choose to migrate if you had the decision to make again?” was asked of every survey participant as a wrap-up question to the interview. ${ }^{64}$ In turn, examining the various possible outcomes related to this question, partially dependent on whether migrants update the reference group to the destination community or hold on to

\footnotetext{
${ }^{63}$ This requirement is conservatively calculated with the first year of migration in mind, when costs are highest due to the cost of the actual trip, $c_{i}$; I assume a 40-hour workweek and 40 weeks of work per year, allowing for periods of unemployment. Given the averages of break-even hours, this means that HIMEX and HIUS migrants have 1237.6 and 1068.32 hours, respectively, left over to cover any uncaptured costs of migration before experiencing zero net gains.

${ }^{64}$ While reporting responses beyond the yes/no answers is outside the scope of this paper, this question resulted in much of the most interesting information that the interviewees shared with me, as many migrants expressed strong mixed feelings on migration related to (dis)advantages of the communities of origin and destination.
} 
the community of origin, allows for inference on the subjective well-being of migrants and further exploration of absolute versus relative gains from migration. ${ }^{65}$

Given that all migrants in the HIMEX and HIUS samples experience both absolute and relative gains (relative to the origin community), if "no" is the chosen answer, only two options exist: (1) the costs of migration are so great that they outweigh the absolute and relative wage gains, or (2) the migrant is indeed updating the reference group and suffering a worse relative standing within the relevant community overshadows the large absolute gains from migration. If the answer is “yes,” one of three explanations must hold for each migrant: (1) updating of the reference group occurs and relative position worsens, however the absolute gains are large enough to outweigh the relative losses, (2) updating of the reference group occurs and relative position improves, adding extra benefit to the absolute gains, or (3) no updating of the reference group occurs, leaving the absolute gains and improved position relative to the origin community.

Exploring the evidence further, even though every single migrant sampled experiences post-migration absolute wage gains and improved position relative to the origin community, migrants are generally worse off post-migration relative to the US destination community than in the Mexican origin community pre-migration. Using the corresponding Public Use Microdata Areas (PUMA) from 2011 American Community Survey data as the relevant US destination community, the average HIUS migrant falls 8 percentiles in the wage distribution to the 7 th percentile. This average disguises a considerable range of outcomes, spread from gains of 11 percentiles to losses of 72

\footnotetext{
${ }^{65}$ It is clearly possible that migrants may have hybrid reference groups, some mixture of both destination and origin communities, however I assume away this possibility in the following discussion for ease of exposition.
} 
percentiles, associated with relative positions ranging from the 1st percentile to the 39th in the US PUMA wage distribution. With 86\% of HIUS answers and 91\% of HIMEX answers “yes,” option (2) can be eliminated as a general explanation; either absolute gains outweigh the relative losses (if reference groups are updated) or migrants overwhelmingly are not updating reference groups. However, it should be pointed out that even though worse relative position in the destination community does not appear to be a deciding factor, a larger fall in relative position may contribute to "no" answers, as a t-test for differences in changes in relative positioning is statistically significant at the $5 \%$ level when comparing the "yes" and "no" responders. Although both groups lose in relative position on average, the "no" responders fall an average of nearly 17 percentiles more than "yes" responders. ${ }^{66}$

Further insight is provided by examining how the answers relate to the size of wage gains and length of time since the migration occurred. Intuitively, one clearly expects subjective well-being (the probability of a "yes" answer) to be increasing in the size of absolute and/or relative wage gains. As far as the length of time, it is not clear whether a longer time at destination would translate into a higher or lower probability of higher subjective well-being; as migrants spend more time in the destination community, it is more likely that they update the reference group to the destination, giving a higher group of incomes with which to compare relative position, however the very fact that more time has been spent since migration could reflect a type of selection in the sense that only the most satisfied migrants remain in the destination community for longer periods of time.

\footnotetext{
${ }^{66}$ These ideas provide avenues for future research, however further data is clearly required in order to reach definitive conclusions.
} 
Looking to the data, Table 3.4 summarizes a comparison of the three main measures of wage gains (without costs) between the migrants answering “yes” and those answering "no" in each sample. I report the difference in means (the means of "no" responders subtracted from the means of "yes" responders) and the standard deviation of wage differences, wage ratios, and percentile changes within the origin wage distributions, as well as the t-statistics, checking to verify if the two groups exhibit statistically significant differences in wage gains. While HIUS migrants certainly provide evidence in favor of the hypothesized positive association between wage gains and subjective well-being, with statistically significant positive differences in means at the $5 \%$ level for both wage differences and percentile changes, HIMEX does not signal the same. Differences in means, while not statistically significant, are actually negative for all three measures, with the unexpected result that migrants answering "no" experience more wage gains on average than the rest of the sample. On the other hand, the very reason for separating the HIMEX and HIUS samples in reporting results, the difference in geographic location of the interviews, may suggest a reason for the HIMEX negative differences in means. The HIMEX sample includes return migrants, who may have achieved relative success through migration and already reached a target goal allowing them to return to the place of origin. If this is indeed the case, some may be unduly influenced by this fact in answering the question as to subjective well-being, given that the return decision was previously made, thereby leading to "no" responders being among the most successful as far as gains from migration.

Switching to net gains, the last row of each section in Table 3.4 reports the same statistics as those above, however using break-even hours $\frac{c_{i}}{D_{W i}}$ as the relevant measure of gains from 
migration. Similar to the wage gains (without costs) story, the HIUS sample supports the hypothesized relationship, showing that "yes" responders have a highly statistically significant lower amount of break-even hours, however the HIMEX sample shows no statistically significant differences, the difference in means being positive ("no" responders have fewer break-even hours than "yes" responders). Table 3.5 displays a similar comparison between the two groups for the length of time in the destination community (length of time since migration). As expected, these results are even more inconclusive, with neither sample providing statistically significant differences between "yes" and "no" responders.

Comparing with an established program of successful development

If we indeed accept migration as not an alternative to economic development, but rather a crucial component of development, it is inevitable to ask how the net gains from migration compare with those of other programs of economic development. In the context of Mexico, a program that has been deemed as one of the most successful is that of PROGRESA/Oportunidades. With no fewer than 30 countries now adopting and including conditional cash transfer programs such as PROGRESA/Oportunidades as part of social policy, it is no surprise to find a return of over 50 papers from a simple title search on "Progresa" at the online economics database at ideas.repec.org. Behrman et al. (2011) provides a follow-up to previous work analyzing the success of the program in Mexico, estimating the "long-run" costs and benefits to the program based on the return in terms of increased schooling due to program participation, as well as various assumed rates of return to schooling and discount rates. Given the lowest assumed discount rate of 3\%, along with the highest assumed return to schooling at 10\%, a 9-10 year-old boy starting six years of 
PROGRESA/Oportunidades participation can expect \$3557 in extra lifetime earnings due to the improved human capital.

While this amount certainly reflects successful outcomes for participants in PROGRESA/Oportunidades, how does it compare to the gains from migration? Even by stacking the deck against migration and employing the highest average break-even hours, 531.68 of the HIUS sample, an astounding result obtains. Converting to $\mathrm{P} \$$, $\$ 3557$ becomes P\$5487, meaning that a Mexican migrant would have to work just over 1000 hours in the United States to match a lifetime of extra earnings from six years of participation in PROGRESA/Oportunidades. Adding this to the work required to recuperate the explicit cost of migration, and assuming a 40-hour workweek, 39 weeks of work in the US surpasses the lifetime net benefits of one of the most successful development programs on record. ${ }^{67}$ While $\mathrm{I}$ do not want to suggest a lack of importance of PROGRESA/Oportunidades or any other development program already in place, this stark comparison clearly emphasizes just how powerful a development tool migration has been and could potentially be in the future.

\section{Observable characteristics and (net) gains}

As a final examination of the survey data, I characterize the relationship between relevant observable traits of the migrant samples and the associated (net) gains of migration. In doing this, I focus on education, gender, time since migration, age at migration, and legal migration status, respectively, and the connection with my various

\footnotetext{
${ }^{67}$ I assume that PROGRESA/Oportunidades entails no cost to the individual participant, intentionally ignoring societal costs in making this comparison. While PROGRESA/Oportunidades clearly has a cost of implementation, it is not clear whether further labor mobility would result in increased or decreased costs to society, due to the many effects on both origin and destination countries of migration. A full analysis of this question is beyond the scope of this paper, however provides a clear avenue for further research.
} 
measures of (net) gains, i.e. $D_{W i}, R_{W i}$, and $\frac{c_{i}}{D_{W i}}$. Further understanding of these relationships is important in attempting to identify if certain types of migrants are relatively more or less likely to enjoy larger gains from migration. While the literature tends to signal potential gains increasing in education, hypothesizing a relationship between education and gains is not straightforward across the varied measures. While it may be reasonable to expect this positive relationship to hold when using $D_{W i}$ as the measure of gains, it is also easy to imagine the opposite result if using $R_{W i}$, given the fact that individuals with lower education levels tend to have a lower initial wage before migration. Concerning the other observable traits of focus, I refrain from hypothesizing the correlations given similar confounding issues, however emphasize time since migration given a potential concern. In reporting the results of both HIMEX and HIUS samples, I group together individual migrants who have migrated over a considerable span of years. Even though all gains are reported using only wage immediately pre- and post-migration, if gains to migration are consistently increasing (decreasing) over time, it may be more reasonable for purposes of comparison to separate the samples further into subdivisions according to year of migration.

Table 3.6 details the simple correlations between the selected observable characteristics and the three varied measures of (net) gains for the HIUS sample. Without controlling for other potential determinants of gains, gains from migration are negatively associated with years of education, positively associated with years since migration, and positively associated with cost of migration, the proxy for legal status given the assumption 
that migration without legal documents results in an increased $c_{i} \cdot{ }^{68}$ The relationship with age at migration is unclear, older age negatively associated with the wage difference, while pointing to larger gains using the wage ratio and break-even hours. A t-test separating men and women results in no statistically significant differences across all three measures of gains, men generally enjoying marginally larger gains relative to women. In addition to $c_{i}$, I employ voluntarily-reported results on the payment to a coyote as an alternative proxy for legal status. This is not an exact measure of legal status because no explicit question as to legal status was included in the household survey, however many interviewees voluntarily offered information on the use of a coyote in answering the question on cost of migration. Furthermore, it is also possible that some migrants who cross the border illegally do not employ a coyote. Dividing the sample into those reporting the use of a coyote (irrespective of reported cost) and those who do not, a maximum t-statistic of 0.94 for ttest on the three measures of gains signals a lack of statistically significant differences between the two groups.

The OLS regression results detailed in Table 3.7 provide an alternative perspective as to the relationship between the selected observable characteristics of migrants and their gains from migration. General positive (negative) associations reflect the correlations detailed in Table 3.6, however the estimates are plagued by a lack of statistical significance, only years since migration and education being statistically significant at the $10 \%$ level for $D_{W i}$ and $R_{W i}$, respectively. In summary, while being less educated, being male, and having migrated a longer time ago appear to be associated with larger gains from migration, the

\footnotetext{
${ }^{68}$ The positive association with cost of migration is expected, as this association does not include net gains. This can be translated as higher wage gains needed to compensate the migrant for a higher migration cost.
} 
evidence on specific determinants of these gains is rather inconclusive for the HIUS sample; nearly all estimates reflect statistical insignificance.

\section{Conclusion}

In answering the three central questions stated in the opening section of this paper, the results tell an overwhelmingly straightforward story. With minimum average wage ratios of 3.92 across all examined samples, migrants are experiencing large wage gains by crossing the Mexico-U.S. border. HIMEX and HIUS migrants gain an average of P\$7.06 and P\$5.22 per hour worked, and enjoy average wage ratios of 5.24 and 5.56, respectively. Even given the explicit cost of migration that cuts into these wage gains, HIMEX and HIUS migrants need only an average of 362 and 521 hours of work in the U.S., respectively, before experiencing outright positive gains. 100\% of the approximately 30\% of HIMEX migrants starting below the Mexican poverty line make the jump out of poverty by relocating, even when measured by the more stringent U.S. measure of the poverty line; only 2 of the $60 \%$ of HIUS migrants moving away from poverty in Mexico remain impoverished by U.S. standards. It is indeed difficult or nearly impossible to find evidence of (or even imagine) a specific program of economic development with a success rate similar to that of migration found in this paper; what program can claim such success stories as pulling all impoverished individuals out of poverty (HIMEX) or increasing participants' incomes more than fivefold on average (HIMEX and HIUS)?

In addition to the absolute wage gains, migration also translates into large movements in migrants' relative positions within the origin wage distributions. Before migrating, the average HIMEX migrant resides at the 38th percentile of the origin wage distribution, moving to the 84th percentile immediately after migration; the average HIUS 
migrant moves from the 15th to the 79th percentile. Upon migration, HIMEX migrants on average pass $52 \%$ of the population in the respective origin wage distribution, while HIUS migrants advance past an astounding average of $64 \%$ of the relevant reference population. Furthermore, while $70 \%$ of HIMEX migrants and $91 \%$ of HIUS migrants have premigration wages below the corresponding median income, 100\% and 34\% rise to the very top of the origin wage distributions, jumping to the highest two deciles immediately after migration.

With these impressively large documented gains available to migrants willing and wanting to move across the border, especially as governments either seek to further limit international migration or discuss options for alternative immigration schemes around the world, further examination of the effects of these policies and complete understanding of the motivation for and determinants of migration is essential. In focusing on the economic development consequences of migration, the effect of migration on migrants themselves perhaps the most important effect of all - must not be forgotten and pushed behind other themes such as effects on sending or receiving countries in the international migration and economic development research. Letouzé et al. (2009) states “...that migration is not an important contributor nor hindrance to development...best seen in terms of the expanded opportunities it offers individuals to carry out their life plans.” However, viewed through the lens of the shifted focus of development on people rather than places, migration not only expands opportunities, those very expanded opportunities contribute greatly to what the definition of economic development should be all about. 
Table 3.1. Summary statistics of migrant samples

\begin{tabular}{lllll}
\hline Indicator & Mean & Median & Std. dev. & Min./max. \\
\hline HIMEX $(n=128)$ & & & & \\
Pre-migration wage (P\$/hr.) & 1.87 & 1.79 & 0.69 & $0.84 / 5.77$ \\
Post-migration wage (P\$/hr.) & 8.93 & 7.83 & 3.12 & $5.92 / 24.23$ \\
Age at interview & 38.02 & 37 & 10.23 & $21 / 75$ \\
Gender (m = 1, f = 2) & 1.28 & 1 & 0.45 & $1 / 2$ \\
Years of schooling & 8.80 & 8 & 2.75 & $3 / 16$ \\
Years since migration & 11.89 & 12 & 6.61 & $1 / 44$ \\
Remittances (monthly P\$) & 181.25 & 200 & 171.95 & $0 / 1000$ \\
\hline
\end{tabular}

HIUS $(n=69)$ 


\begin{tabular}{lllll} 
Pre-migration wage (P\$/hr.) & 1.75 & 1.49 & 1.25 & $0.25 / 8.57$ \\
Post-migration wage (P\$/hr.) & 6.96 & 6.73 & 1.37 & $4.14 / 14.04$ \\
Age at interview & 40.44 & 39 & 13.55 & $18 / 76$ \\
Gender $(\mathrm{m}=1, \mathrm{f}=2)$ & 1.48 & 1 & 0.50 & $1 / 2$ \\
Years of schooling & 6.65 & 6 & 3.67 & $0 / 14$ \\
Years since migration & 16.77 & 14 & 9.31 & $1 / 45$ \\
Remittances (monthly P\$) & 102.20 & 100 & 82.83 & $0 / 300$ \\
\hline
\end{tabular}


Table 3.2. Migrant wage gains

\begin{tabular}{|c|c|c|c|c|c|c|c|c|c|c|c|}
\hline & & \multicolumn{2}{|c|}{ Pre-migration wage } & \multicolumn{2}{|c|}{ Post-migration wage } & \multicolumn{3}{|c|}{ Wage difference } & \multicolumn{3}{|c|}{ Wage ratio } \\
\hline & & Average & Median & Average & Median & Average & Median & Std. dev & Average & Median & Std. dev. \\
\hline \multicolumn{2}{|c|}{ HIMEX } & 1.87 & 1.79 & 8.93 & 7.83 & 7.06 & 5.87 & 1.21 & 5.24 & 4.50 & 4.01 \\
\hline \multicolumn{2}{|c|}{ HIUS } & 1.75 & 1.49 & 6.96 & 6.95 & 5.22 & 5.15 & 3.03 & 5.56 & 4.29 & 2.36 \\
\hline \multicolumn{12}{|l|}{$\overline{\text { EMIF }}$} \\
\hline \multirow[t]{2}{*}{2009} & full & 2.44 & 2.12 & 10.12 & 9.36 & 7.68 & 7.12 & 4.08 & 5.29 & 4.68 & 3.13 \\
\hline & $<12$ months & 2.55 & 2.11 & 9.69 & 8.67 & 7.14 & 6.73 & 4.16 & 4.69 & 4.10 & 2.68 \\
\hline \multirow[t]{2}{*}{2008} & full & 2.99 & 2.32 & 10.62 & 9.36 & 7.62 & 6.92 & 5.40 & 4.73 & 3.99 & 3.12 \\
\hline & $<12$ months & 3.63 & 2.61 & 10.05 & 9.10 & 6.42 & 6.58 & 3.94 & 3.92 & 3.59 & 2.12 \\
\hline \multirow[t]{2}{*}{2007} & full & 2.91 & 2.20 & 10.34 & 9.00 & 7.43 & 6.56 & 5.26 & 4.87 & 4.13 & 3.47 \\
\hline & $<12$ months & 3.55 & 2.44 & 10.36 & 8.64 & 6.82 & 6.25 & 4.78 & 4.21 & 3.69 & 2.55 \\
\hline \multirow[t]{2}{*}{2006} & full & 3.09 & 2.23 & 10.06 & 8.96 & 6.97 & 6.45 & 5.22 & 4.75 & 4.03 & 3.70 \\
\hline & $<12$ months & 3.30 & 2.27 & 10.06 & 8.96 & 6.76 & 5.95 & 6.26 & 4.56 & 3.74 & 3.91 \\
\hline \multirow[t]{2}{*}{2005} & full & 2.92 & 2.35 & 10.03 & 9.20 & 7.10 & 6.44 & 5.07 & 4.84 & 3.85 & 3.57 \\
\hline & $<12$ months & 3.06 & 2.39 & 10.08 & 8.63 & 7.01 & 6.33 & 5.29 & 4.55 & 3.74 & 3.52 \\
\hline \multirow[t]{2}{*}{2004} & full & 2.76 & 2.07 & 9.68 & 8.93 & 6.92 & 6.26 & 4.55 & 4.83 & 4.05 & 3.15 \\
\hline & $<12$ months & 2.78 & 2.07 & 9.39 & 8.33 & 6.61 & 6.08 & 4.82 & 4.54 & 3.74 & 2.94 \\
\hline \multirow[t]{2}{*}{2003} & full & 2.90 & 2.24 & 9.10 & 8.54 & 6.20 & 6.00 & 4.38 & 4.65 & 3.92 & 3.42 \\
\hline & $<12$ months & 3.04 & 2.32 & 8.91 & 8.24 & 5.87 & 5.69 & 4.12 & 4.22 & 3.64 & 3.23 \\
\hline \multirow[t]{2}{*}{2002} & full & 2.81 & 2.12 & 9.16 & 8.75 & 6.35 & 6.17 & 4.28 & 4.85 & 3.93 & 3.57 \\
\hline & $<12$ months & 2.98 & 2.12 & 8.86 & 8.52 & 5.89 & 5.91 & 4.11 & 4.56 & 3.93 & 3.34 \\
\hline \multirow[t]{2}{*}{2001} & full & 2.67 & 1.98 & 9.18 & 7.94 & 6.51 & 6.18 & 4.77 & 5.14 & 4.54 & 3.67 \\
\hline & $<12$ months & 2.66 & 2.01 & 9.01 & 7.83 & 6.36 & 5.94 & 4.81 & 4.73 & 4.21 & 3.11 \\
\hline \multirow[t]{2}{*}{2000} & full & 2.42 & 1.79 & 8.81 & 7.86 & 6.39 & 6.17 & 3.71 & 5.27 & 4.65 & 3.30 \\
\hline & $<12$ months & 2.48 & 1.79 & 8.66 & 7.86 & 6.19 & 6.12 & 3.50 & 5.00 & 4.50 & 3.05 \\
\hline \multirow[t]{2}{*}{1999} & full & 2.56 & 1.80 & 9.13 & 8.10 & 6.57 & 6.07 & 5.06 & 5.29 & 4.50 & 3.52 \\
\hline & $<12$ months & 2.50 & 1.80 & 8.97 & 8.10 & 6.47 & 6.00 & 4.83 & 5.22 & 4.43 & 3.53 \\
\hline
\end{tabular}


Table 3.3. Break-even hours

\begin{tabular}{lllll}
\hline & Mean & Std. dev. & Median & Min./max. \\
\hline HIMEX & & & & \\
Break-even hours $\left(\frac{c_{i}}{D_{W i}}\right)$ & 362.40 & 194.43 & 341.11 & $36.50 / 1104.62$ \\
\hline $\begin{array}{l}\text { HIUS } \\
\text { Break-even hours }\left(\frac{c_{i}}{D_{W i}}\right)\end{array}$ & 531.68 & 325.58 & 455.44 & $39.50 / 2017.49$ \\
\hline
\end{tabular}

Table 3.4. Wage gains and subjective well-being

\begin{tabular}{llll}
\hline & Mean & Std. dev. & T-stat \\
\hline HIMEX & & & \\
Wage difference & -0.71 & 1.01 & -0.71 \\
Wage ratio & -1.37 & 0.77 & -1.77 \\
Percentile change (origin) & -10.11 & 6.76 & -1.49 \\
Break-even hours & 81.89 & 64.99 & 1.26 \\
\hline HIUS & & & \\
Wage difference & 1.09 & 0.42 & 2.60 \\
Wage ratio & 1.92 & 1.44 & 1.34 \\
Percentile change (origin) & 17.82 & 6.32 & 2.82 \\
Break-even hours & -354.40 & 125.66 & -2.82 \\
\hline
\end{tabular}

Notes: Mean reports the difference in means between the respective values of "yes" responders minus those of "no" responders, while std. dev. is the associated standard deviation. T-stat signals the statistical significance of the differences across the two groups, an absolute value greater than 1.96 being statistically significant at the $5 \%$ level.

Table 3.5. Length of time since migration and subjective well-being

\begin{tabular}{llll}
\hline & Mean & Std. dev. & T-stat \\
\hline HIMEX & & & \\
Length of time since migration & -0.74 & 2.17 & -0.34 \\
\hline $\begin{array}{l}\text { HIUS } \\
\text { Length of time since migration }\end{array}$ & 0.98 & 3.37 & 0.29 \\
\hline
\end{tabular}

Notes: Mean reports the difference in means between the respective values of "yes" responders minus those of "no" responders, while std. dev. is the associated standard deviation. T-stat signals the statistical significance of the differences across the two groups, an absolute value greater than 1.96 being statistically significant at the $5 \%$ level. 
Table 3.6. Simple correlations: observable characteristics and gains from migration

\begin{tabular}{lllll}
\hline & Years of education & Years since migration & Age at migration & $c_{i}$ \\
\hline$D_{W i}$ & -0.16 & 0.47 & -0.02 & 0.18 \\
$R_{W i}$ & -0.39 & 0.07 & 0.22 & 0.12 \\
$\left(\frac{c_{i}}{D_{W i}}\right)$ & 0.06 & -0.24 & -0.01 & --- \\
\hline
\end{tabular}

Table 3.7. OLS regression results

\begin{tabular}{lllllll}
\hline Dependent variable: & \multicolumn{2}{c}{$D_{W i}$} & \multicolumn{2}{c}{$R_{W i}$} & \multicolumn{2}{c}{$c_{i}$} \\
Covariates & Coefficient & Std. error & Coefficient & Std. error & Coefficient & Std. error \\
\hline Education & -0.03 & 0.05 & $-0.47^{* *}$ & 0.20 & -0.37 & 16.76 \\
Gender & -0.21 & 0.28 & 0.32 & 1.12 & 12.50 & 94.75 \\
Years since migration & $0.05^{* * *}$ & 0.02 & -0.00 & 0.06 & -8.88 & 5.31 \\
Age at migration & -0.00 & 0.02 & 0.01 & 0.07 & -0.96 & 5.97 \\
Cost & 0.00 & 0.00 & 0.00 & 0.00 & -- & --- \\
Constant & $4.78^{* * *}$ & 0.90 & $6.93^{*}$ & 3.66 & $631.95^{* *}$ & 296.35 \\
$R^{2}$ & 0.27 & --- & 0.17 & --- & 0.06 & --- \\
\hline
\end{tabular}

Notes: ${ }^{*}, * *$ and ${ }^{* * *}$ denote significance at the $10 \%, 5 \%$, and $1 \%$ levels, respectively. 
Figure 3.1. HIMEX wages before and after migration

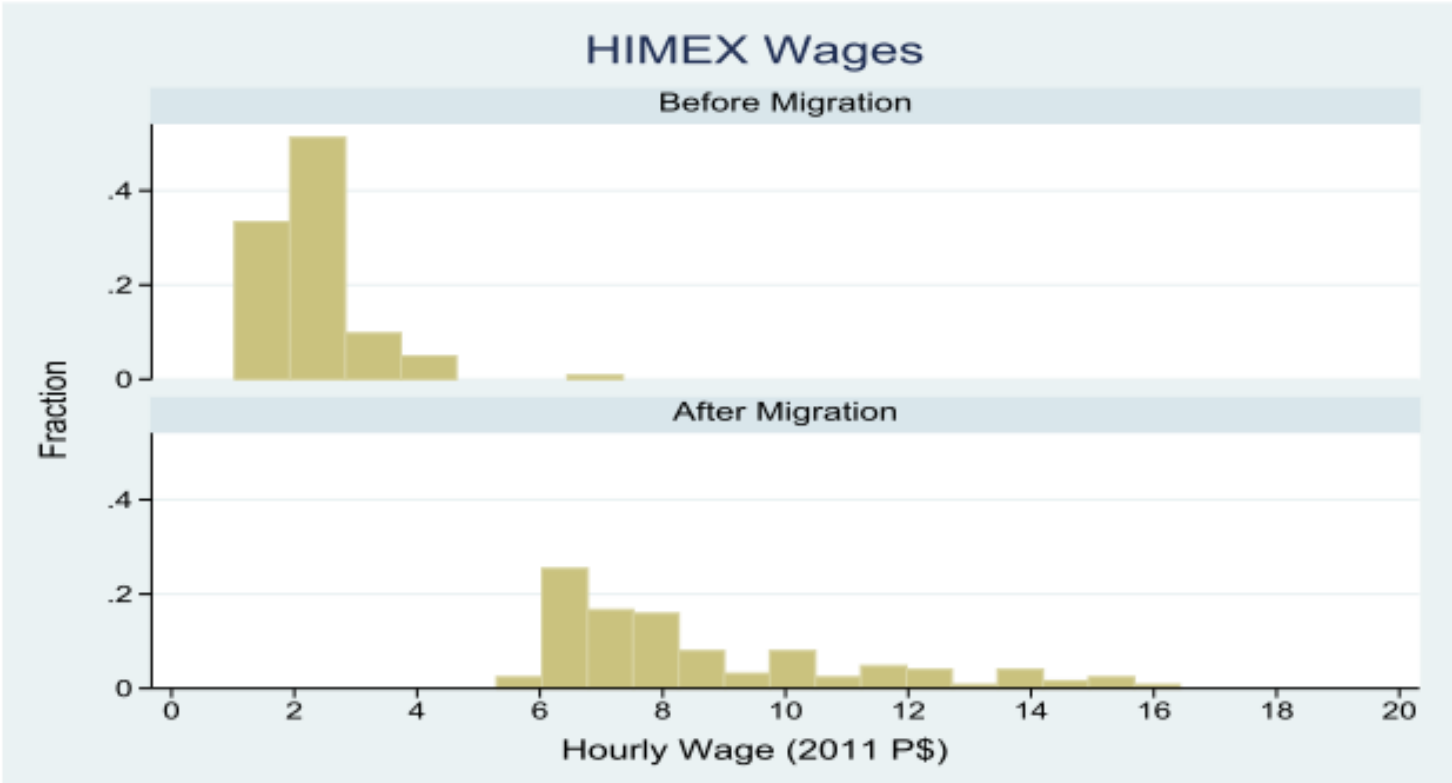

Figure 3.2. HIMEX wage gains from migration

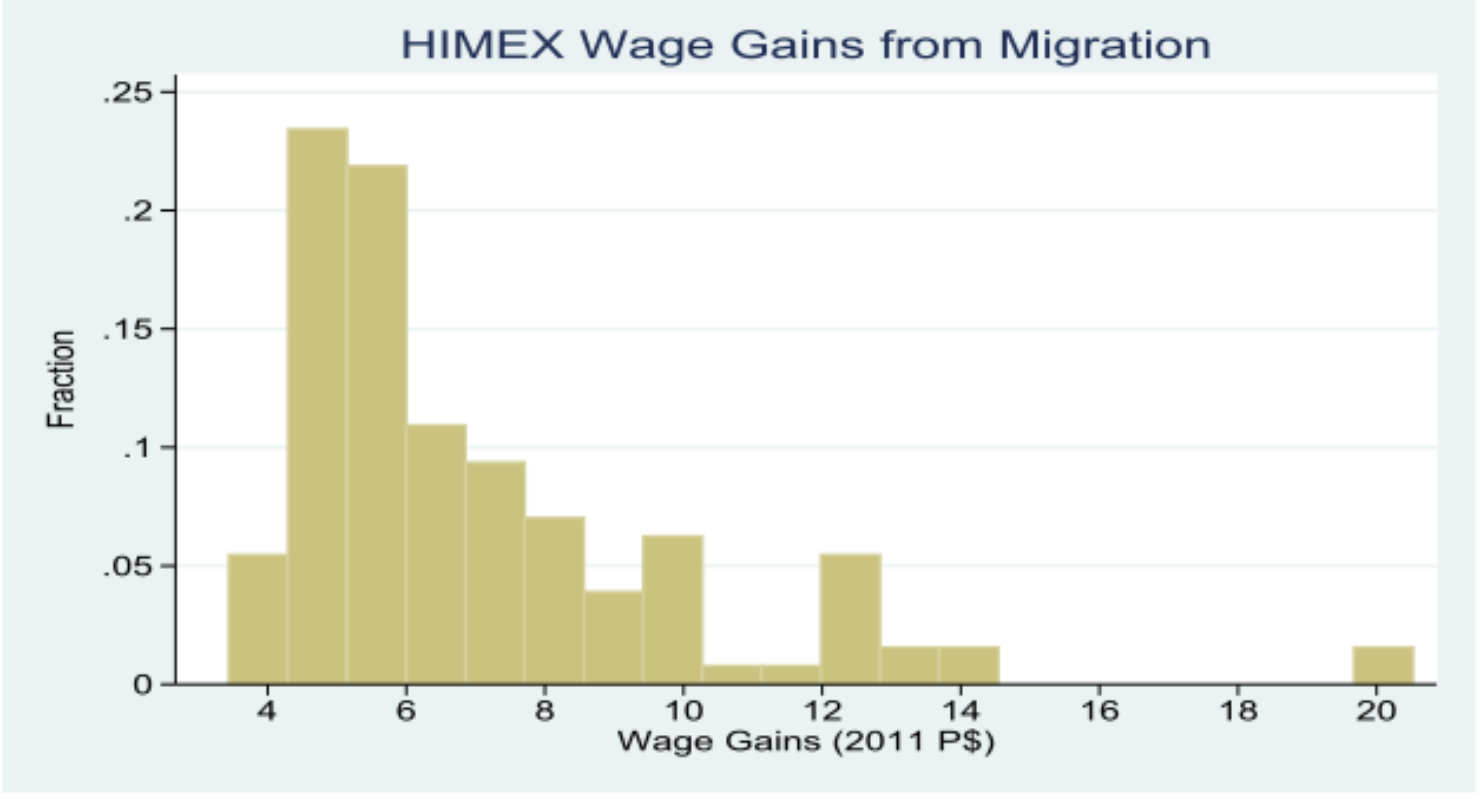


Figure 3.3. HIMEX wage ratios

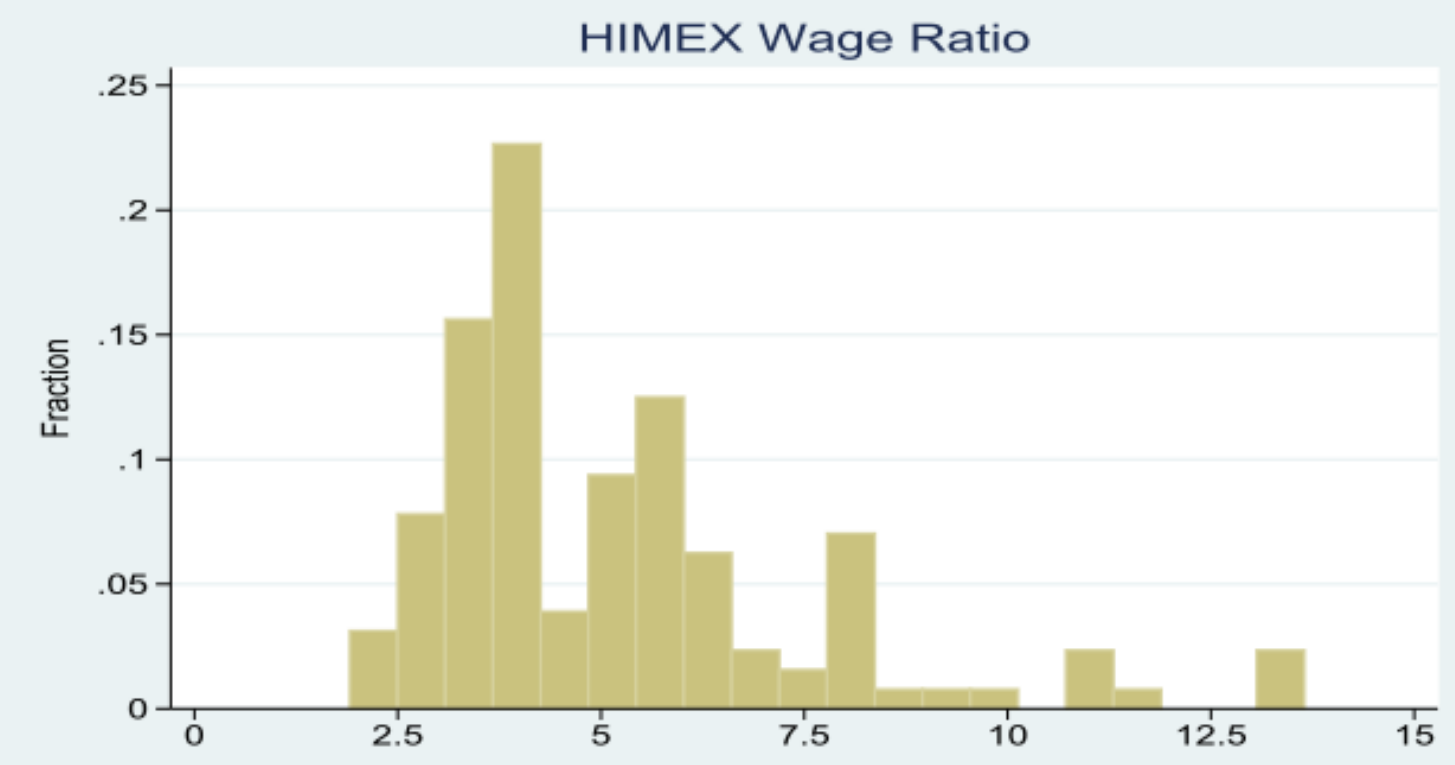

Figure 3.4. HIUS wages before and after migration

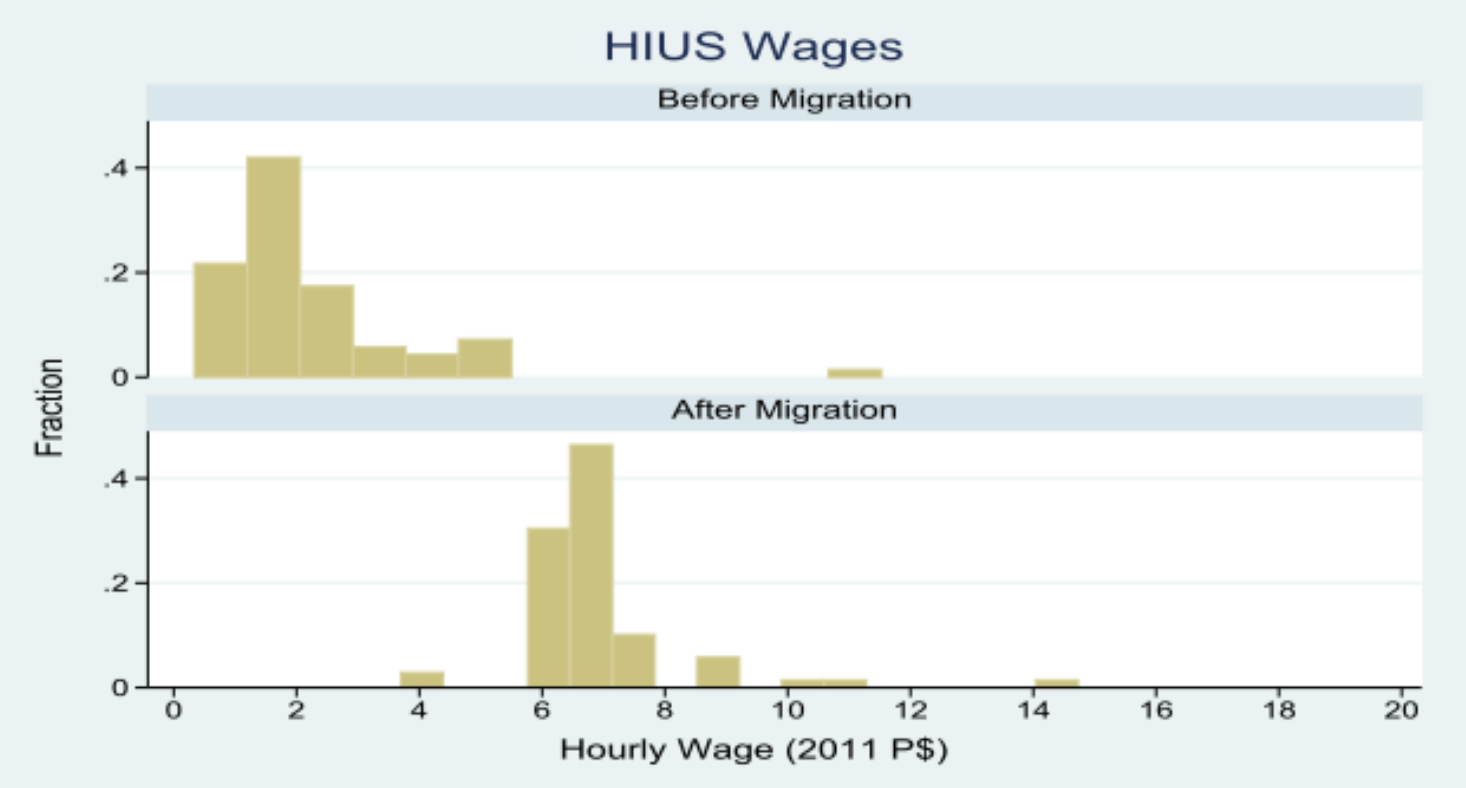


Figure 3.5. HIUS wage gains from migration

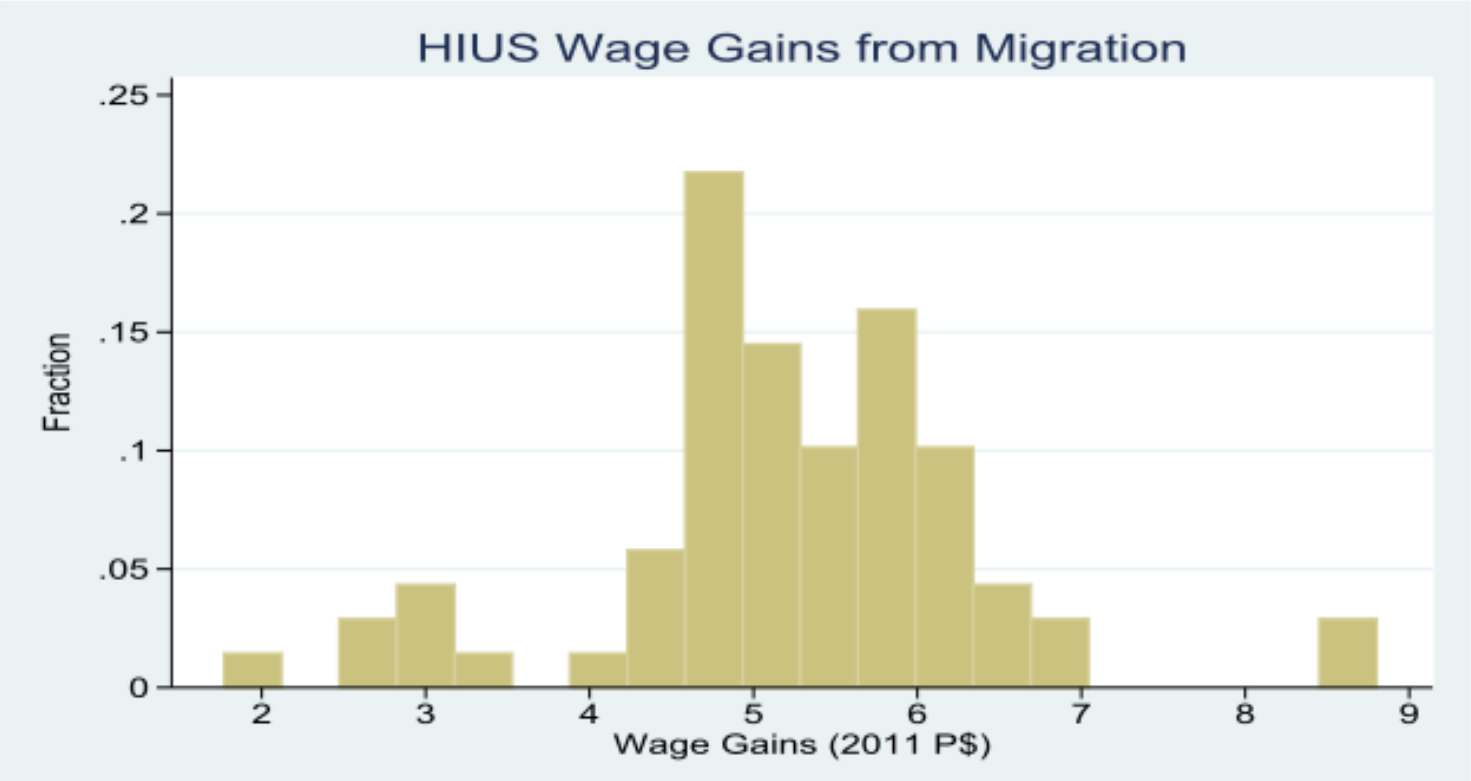

Figure 3.6. HIUS wage ratios

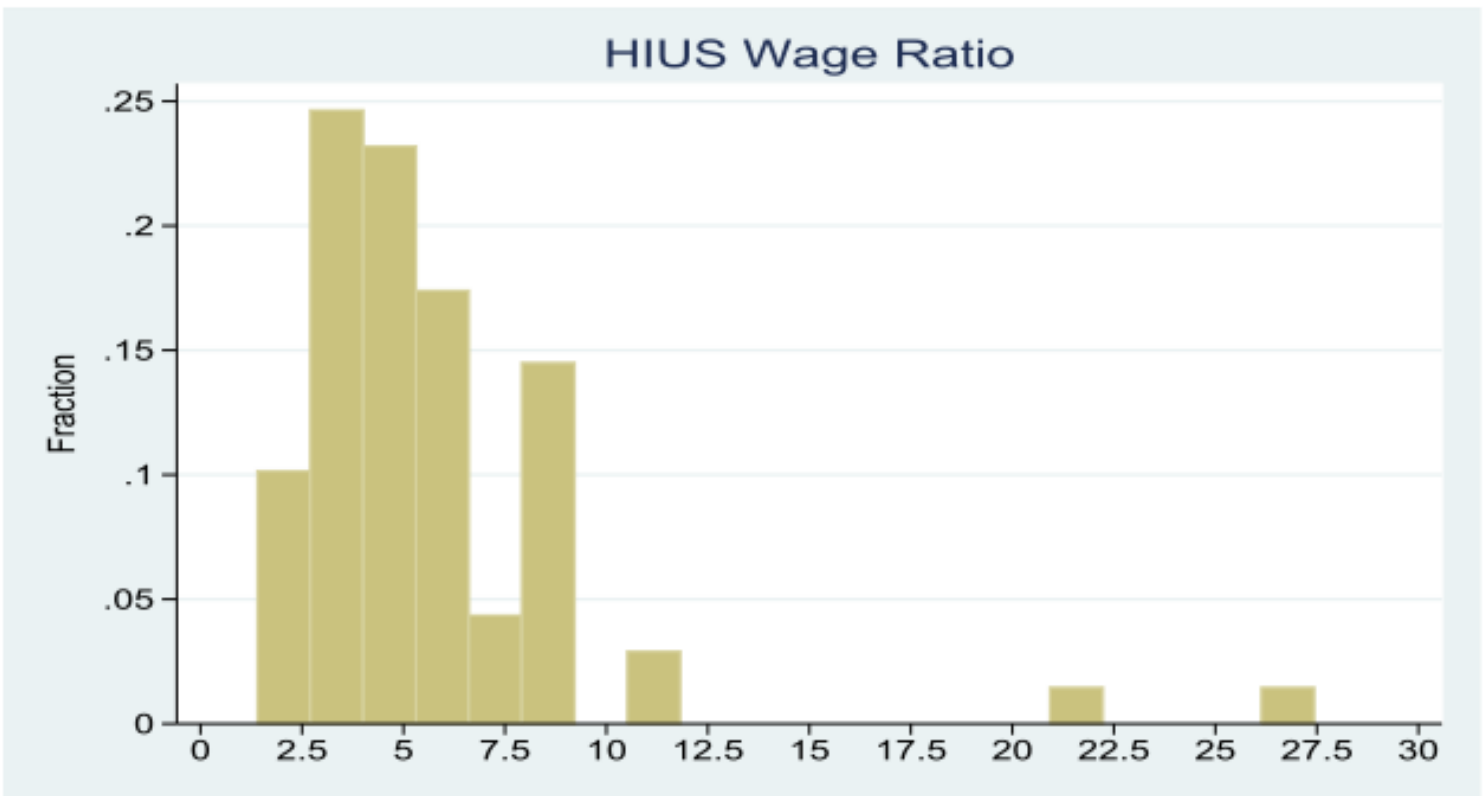




\section{Figure 3.7. EMIF wages}

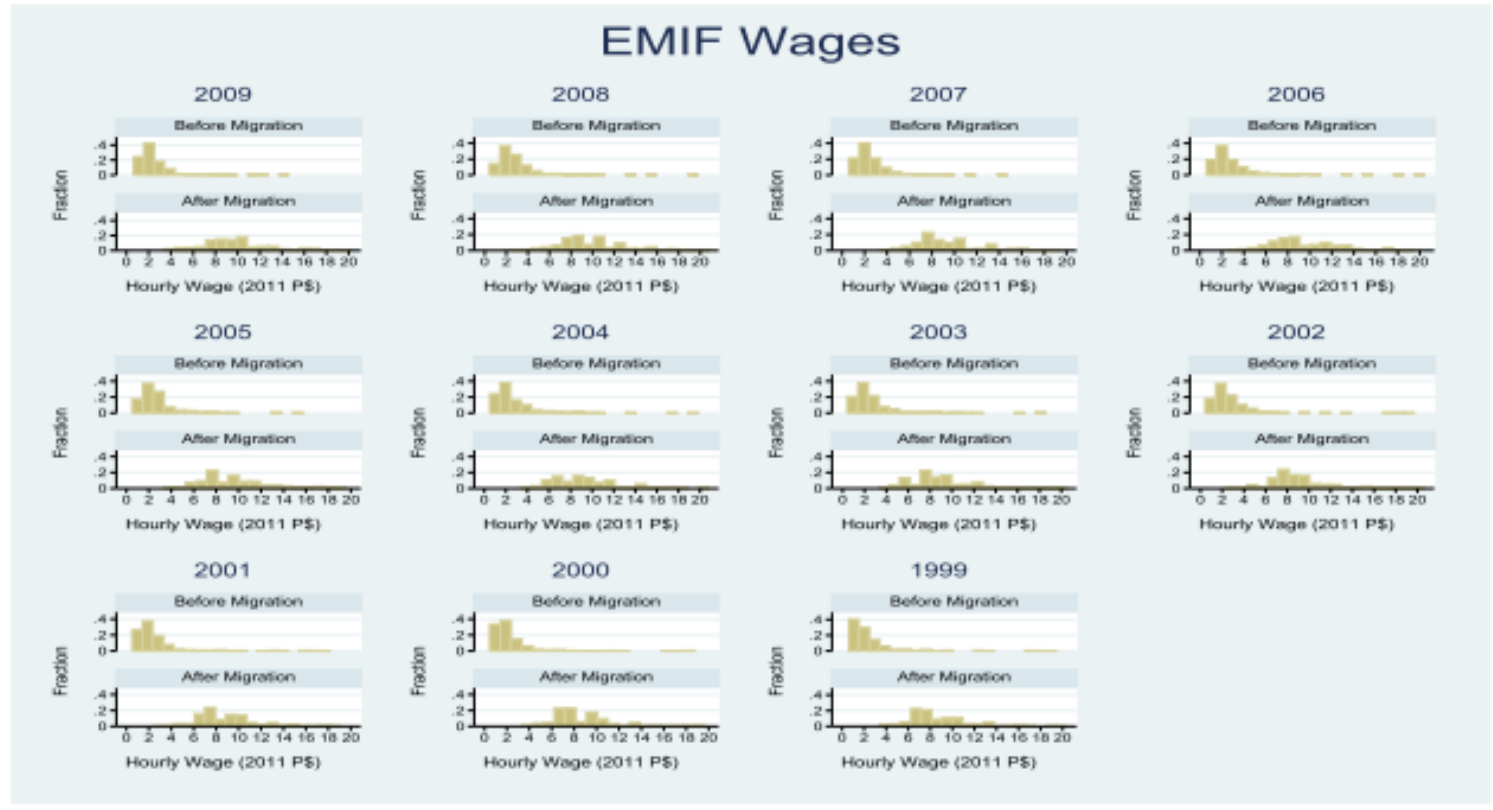

Figure 3.8. EMIF wage ratios
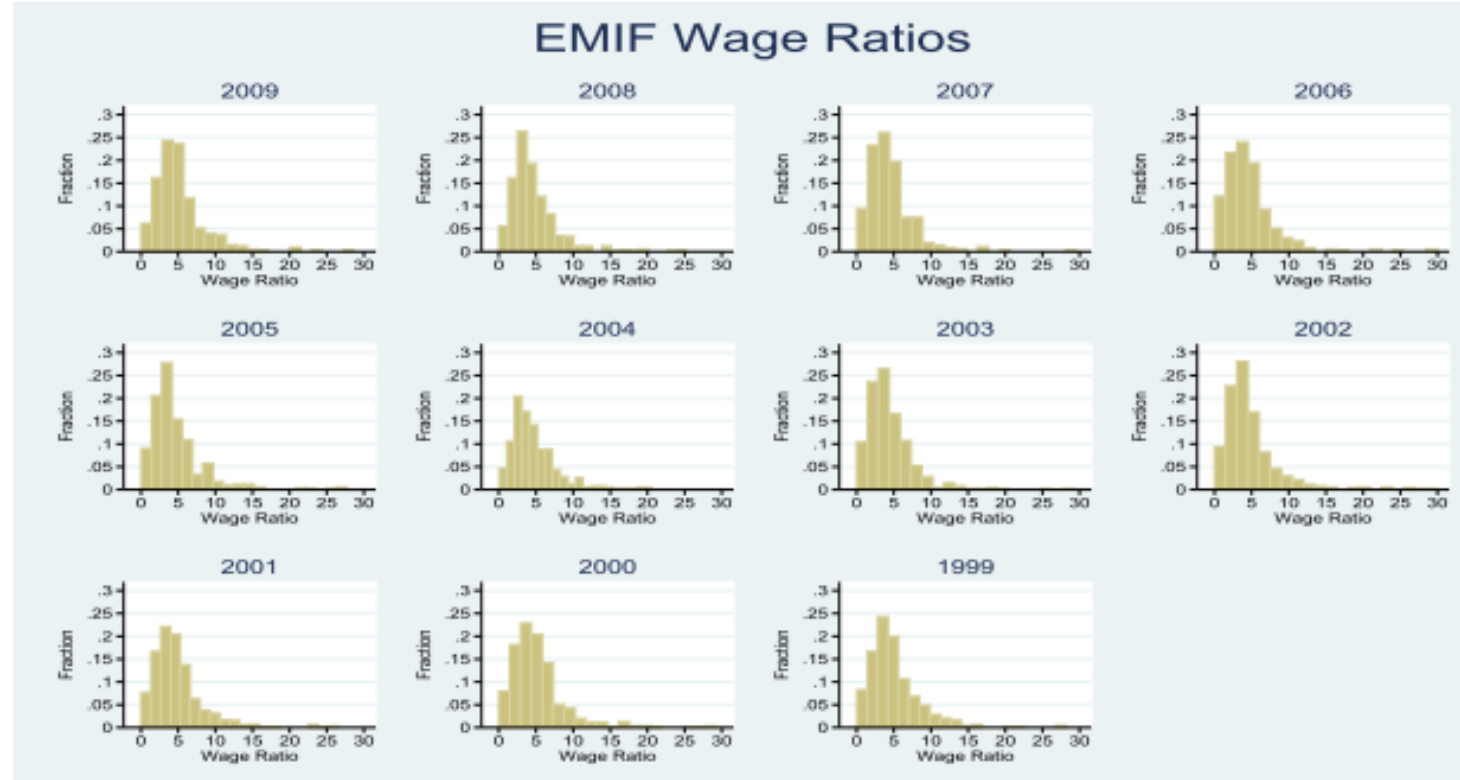
Figure 3.9. HIMEX wages

HIMEX Wages

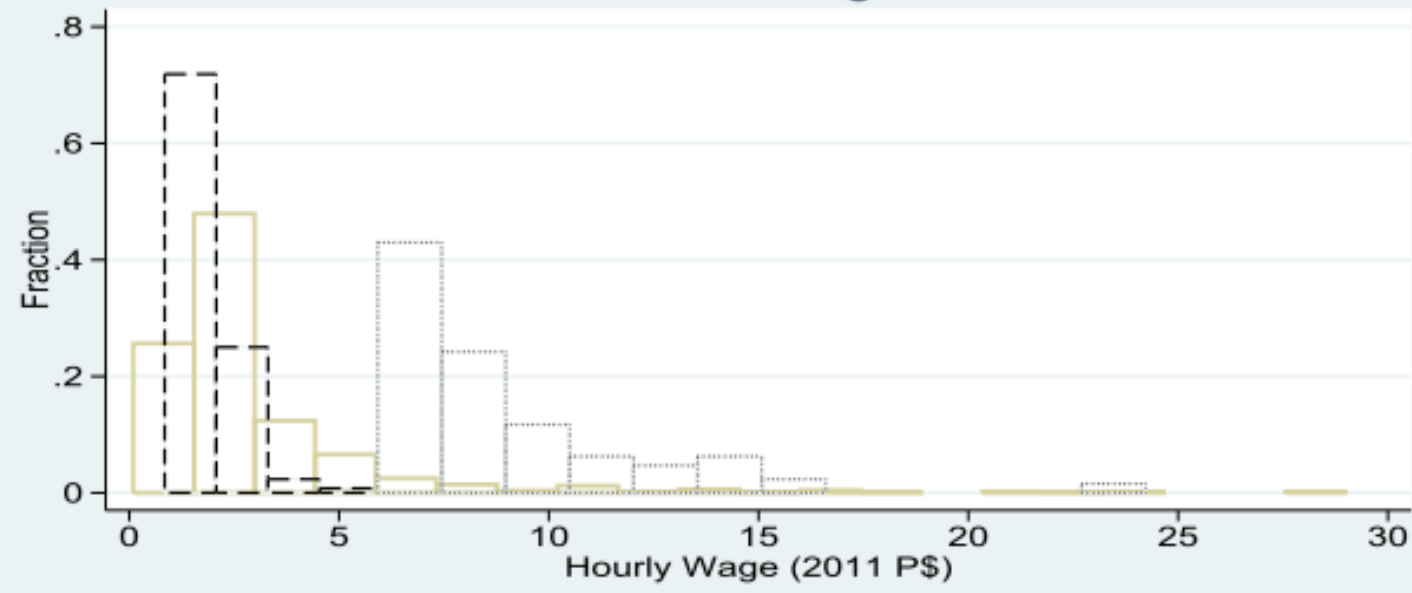

Solid bars: Census

Dotted bars: HIMEX post-migration

Dashed bars: HIMEX pre-migration

Figure 3.10. HIMEX in Census distribution

HIMEX in Census Distribution

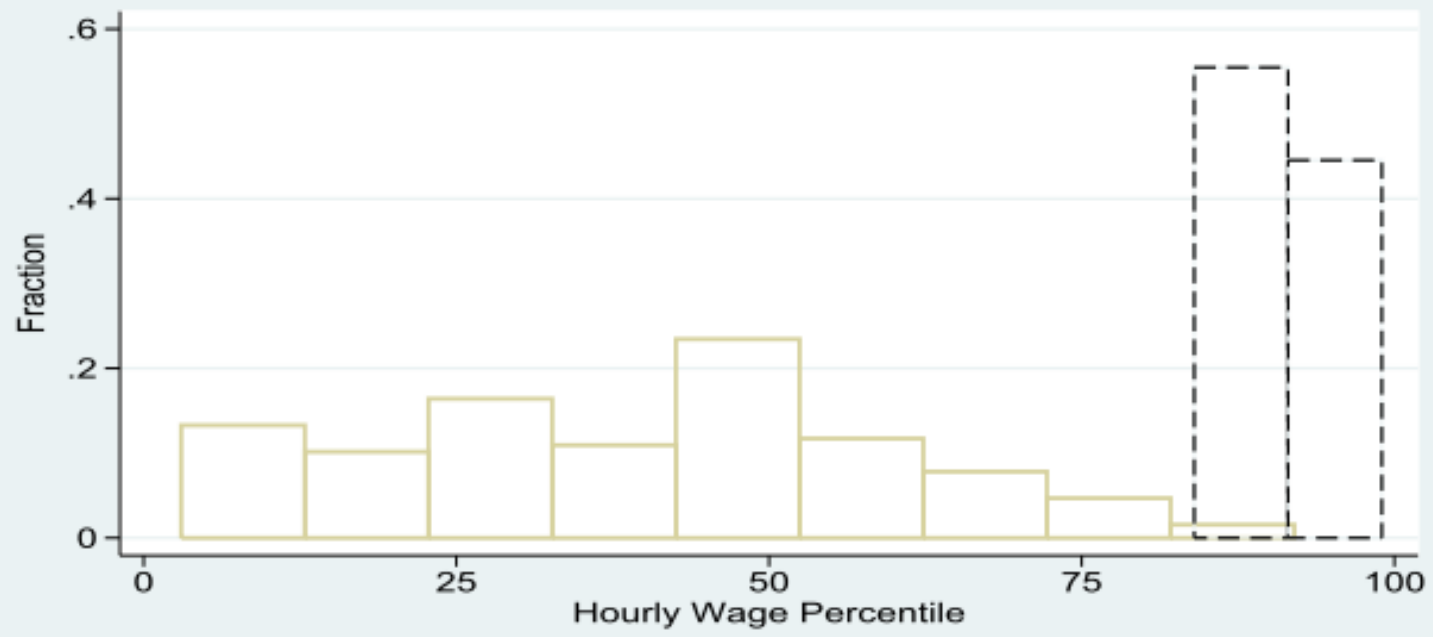

Solid bars: Pre-migration

Dashed bars: Post-migration 
Figure 3.11. HIUS in Census distribution

HIUS in Census Distribution

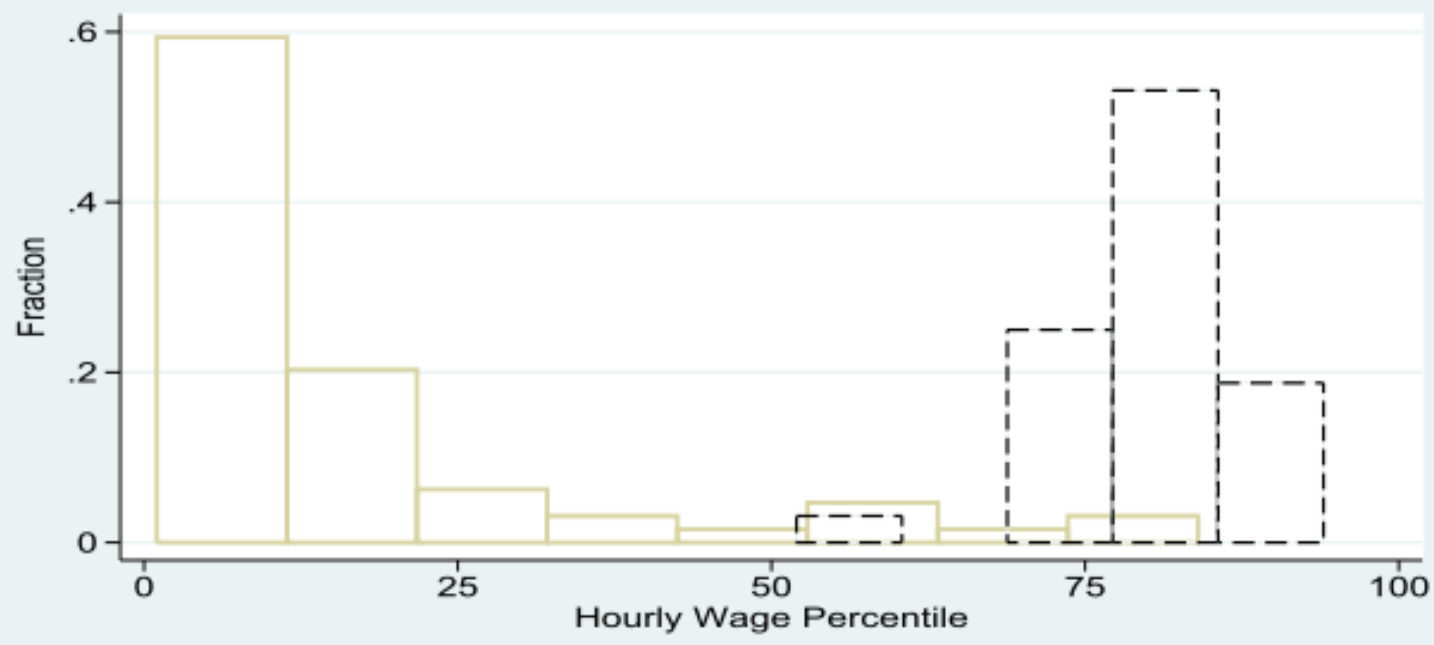

Solid bars: Pre-migration

Dashed bars: Post-migration 


\section{LIST OF REFERENCES}

Aleksynska, M. and G. Peri (2011). Isolating the network effect of immigrants on trade, Working Paper 2011/26, CEPII.

Amuedo-Dorantes, C. and S. de la Rica (2007). Immigrants' responsiveness to labor market conditions and their impact on regional employment disparities: evidence from Spain, Working Papers 2007-34, FEDEA.

Anderson, J. and E. van Wincoop (2003). Gravity with gravitas: a solution to the border puzzle, American Economic Review, 93 (1): 170-192.

Artal-Tur, A., Pallardo-López, V., and F. Requena-Silvente (2012). The trade-enhancing effect of immigration networks: New evidence on the role of geographic proximity, Economics Letters, 116 (3): 554-557.

Bartram, D. (2011). Economic migration and happiness: comparing immigrants' and natives' happiness gains from income, Social Indicators Research, 103 (1), 57-76.

Behrman, J., Parker, S. and P. Todd (2011). Do conditional cash transfers for schooling generate lasting benefits? A five-year followup of PROGRESA/Oportunidades, Journal of Human Resources, 46 (1): 93-122.

Bertrand, M., Duflo, E., and S. Mullainathan (2004). How much should we trust difference-in-differences estimates?, Quarterly Journal of Economics, 119: 249-75.

Borjas, G. (2006). Native internal migration and the labor market impact of immigration, Journal of Human Resources, 41: 221-58.

Borjas, G., Grogger, J., and G. Hanson (2008). Imperfect substitution between immigrants and natives: a reappraisal, NBER Working Paper No. 13887.

Borjas, G., Grogger, J., and G. Hanson (2011). Substitution between immigrants, natives, and skill groups, NBER Working Paper No. 17461.

Borjas, G., Freeman, R., and L. Katz (1997). How much do immigration and trade affect labor market outcomes?, Brookings Papers on Economic Activity, 1: 1-67.

Bureau of Consular Affairs (2012). http://travel.state.gov/visa/bulletin/bulletin_1360.html (accessed 1 September 2013).

Canavire Bacarreza, G. and L. Ehrlich (2006). The impact of migration on foreign trade: a developing country approach, MPRA Paper 1090, University Library of Munich. 
Caponi, V. and M. Plesca (2012). Empirical characteristics of legal and illegal immigrants in the US, mimeo, Ryerson University.

Card, D. (2001). Immigrant inflows, native outflows, and the local labor market impacts of higher immigration, Journal of Labor Economics, 19: 22-64.

Card, D. (2009). Immigration and inequality, American Economic Review, 99: 1-21.

Card, D. and J. DiNardo (2000). Do immigrant inflows lead to native outflows?, American Economic Review, 90: 360-67.

Card, D. and E. Lewis (2007). The diffusion of Mexican immigrants during the 1990s: explanations and impacts, in Mexican Immigration to the United States, Ed. G. Borjas, University of Chicago Press, Chicago, pp. 193-227.

Card, D., Dustmann, C. and I. Preston (2012). Immigration, wages, and compositional amenities, Journal of the European Economic Association, 10 (1): 78-119.

Caselli, F. and J. Feyrer (2007). The marginal product of capital, Quarterly Journal of Economics, 122 (2): 535-65.

Clemens, M. and L. Pritchett (2008). Income per natural: measuring development as if people mattered more than places, Working Papers 143, Center for Global Development.

Clemens, M., Montenegro, C. and L. Pritchett (2008). The place premium: wage differences for identical workers across the U.S. border, Working Papers 148, Center for Global Development.

Consejo Nacional de Evaluación de la Política de Desarrollo Social (2012). http://www.coneval.gob.mx/cmsconeval/rw/pages/medicion/faq.es.do;jsessionid $=69339 \mathrm{e} 325 \mathrm{~b} 9 \mathrm{e} 2605 \mathrm{eab} 24 \mathrm{~d} 81098 \mathrm{e} 5 \mathrm{e} 0 \mathrm{e} 66 \mathrm{c} 506249 \mathrm{~b} 18 \mathrm{eb} 7 \mathrm{e} 8 \mathrm{f} 4018 \mathrm{~b} 0 \mathrm{edc} 325 \mathrm{~d} 4 . \mathrm{e} 34 \mathrm{QaN} 4$ LaxeOa40Qaxf0 URL (accessed 12 December 2012).

di Giovanni, J., Levchenko, A. and F. Ortega (2013). A global view of cross-border migration, IZA Discussion Papers 6584, Institute for the Study of Labor.

Donald, S. and K. Lang (2007). Inference with difference-in-differences and other panel data, The Review of Economics and Statistics, 89: 221-33.

Egger, P., von Ehrlich, M., and D. Nelson (2012). Migration and trade, World Economy, 35 (2): 216-241.

Felbermayr, G. and B. Jung (2009). The pro-trade effect of the brain drain: sorting out confounding factors, Economics Letters, 104: 72-75. 
Felbermayr, G. and F. Toubal (2012). Revisiting the trade-migration nexus: evidence from new OECD data, World Development, 40 (5): 928-937.

Filer, R. (1992). The impact of immigrant arrivals on migratory patterns of native workers, in Immigration and the Workforce: Economic Consequences for the United States and Source Areas, (Eds) G. Borjas and R. Freeman, University of Chicago Press, Chicago, pp. 245-69.

Frey, W. (1995). Immigration and internal migration "flight" from US metro areas: toward a new demographic balkanization, Urban Studies, 32: 733-57.

Girma, S. and Z. Yu (2002). The link between immigration and trade: evidence from the United Kingdom, Weltwirtschaftliches Archiv, 138 (1): 115-130.

Gould, D. (1994). Immigrant links to the home country: empirical implications for US bilateral trade flows, The Review of Economics and Statistics, 76 (2): 302-316.

Hamilton, B. and J. Whalley (1984). Efficiency and distributional implications of global restrictions on labour mobility: calculations and policy implications, Journal of Development Economics, 14 (1-2): 61-75.

Hanson, G. (2010). International migration and the developing world, in Handbook of Development Economics, Chapter 66, pp. 4363-4414.

Hatzigeorgiou, A. and M. Lodefalk (2011). Trade and migration: firm-level evidence, Working Paper 2011/6, Swedish Business School.

Head, K. and J. Ries (1998). Immigration and trade creation: econometric evidence from Canada, Canadian Journal of Economics, 31 (1): 47-62.

Herander, M. and Saavedra, L. (2005). Exports and the structure of immigrant-based networks: the role of geographic proximity, The Review of Economics and Statistics, 87 (2): 323-335.

Instituto de los Mexicanos en el Exterior (2013). http://www.ime.gob.mx/index.php? option=com_content\&view=article\&id=19\&Itemid=507\&lang=es (accessed 23 January 2013).

Instituto Nacional de Estadística y Geografía (2013). http://www.inegi.org.mx/sistemas/ mexicocifras/default.aspx?e=1 (accessed 23 January 2013).

Instituto Nacional de Estadística y Geografía (2012). http://www.censo2010.org.mx/ (accessed 12 December 2012). 
Klein, P. and G. Ventura (2004). Do migration restrictions matter? Unpublished manuscript.

Kritz, M. and D. Gurak (2001). The impact of immigration on the internal migration of natives and immigrants, Demography, 38: 133-45.

Kugler, M. and H. Rapoport (2011). Migration, FDI and the margins of trade, CID Working Paper 2011/222, Harvard University.

Letouzé, E., Purser, M., Rodríguez, F., and M. Cummins (2009). Revisiting the migration-development nexus: a gravity model approach," Human Development Research Papers HDRP-2009-44, United Nations Development Programme.

McKenzie, D. and H. Rapoport (2007). Network effects and the dynamics of migration and inequality: theory and evidence from Mexico, Journal of Development Economics, 84: $1-24$.

McKenzie, D., Gibson, J. and S. Stillman (2010). How important is selection? Experimental vs. non-experimental measures of the income gains from migration, Journal of the European Economic Association, 8 (4): 913-45.

Mexican Migration Project (2012). http://mmp.opr.princeton.edu/ (accessed 12 December 2012).

National Conference of State Legislatures (2012). Available at http://www.ncsl.org/ (accessed 4 February 2012).

Ottaviano, G. and G. Peri (2012). Rethinking the effects of immigration on wages, Journal of the European Economic Association, 10: 152-197.

Parsons, C. and P. Vézina (2013). Migrant networks and trade: the Vietnamese boat people as a natural experiment, mimeo, University of Oxford.

Passel, J. and D. Cohn (2009). A portrait of unauthorized immigrants in the United States, Research Report, Pew Hispanic Center.

Peri, G. (2011). Rethinking the area approach: immigrants and the labor market in California, Journal of International Economics, 84: 1-14.

Peri, G. and F. Requeña (2010). The trade creation effect of immigrants: evidence from the remarkable case of Spain, Canadian Journal of Economics, 43 (4): 1433-1459.

Peri, G. and C. Sparber (2008). The fallacy of crowding-out: a note on "Native internal migration and the labor market impact of immigration", mimeo, University of California, Davis. 
Pritchett, L. (2006). Let their people come: breaking the gridlock on global labor mobility, Center for Global Development, Washington D.C.

Raphael, S. and L. Ronconi (2009). The labor market impact of state-level immigration legislation targeted at unauthorized immigrants, mimeo, University of California, Berkeley.

Rauch, J. and V. Trindade (2002). Ethnic Chinese networks in international trade, Review of Economics and Statistics, 84 (1): 116-130.

Robertson, C. (2011). After ruling, hispanics flee an Alabama town, The New York Times, 3 October 2011.

Secretaría del Trabajo y Previsión Social (2012). http://www.colef.net/emif/index.php (accessed 12 December 2012).

Serrano-Domingo, G. and F. Requeña-Silvente (2013). Re-examining the migration-trade link using province data: an application of the generalized propensity score, Economic Modelling, 32: 247-261.

Stark, O. (2009). Reasons for remitting, World Economics, 10 (3): 147-57.

Stark, O. and J. Taylor (1991). Migration incentives, migration types: the role of relative deprivation, The Economic Journal, 101: 1163-78.

Stillman, S., Gibson, J., McKenzie, D., and R. Halahingano (2012). Miserable migrants? Natural experiment evidence on international migration and objective and subjective well-being, IZA Discussion Papers 6871, Institute for the Study of Labor.

Taylor, J. and G. Dyer (2009). Migration and the sending economy: a disaggregated rural economy-wide analysis, Journal of Development Studies, 45 (6): 966-89.

US Bureau of Economic Analysis, 2013. http://www.bea.gov/iTable/iTable.cfm?ReqID =70\&step=1\&isuri=1\&acrdn=1 (accessed 23 January 2013).

Vézina, P. (2012). How migrant networks facilitate trade: evidence from Swiss exports, Swiss Journal of Economics and Statistics, 148 (3): 449-476.

Walmsley, T. and A. Winters (2005). Relaxing the restrictions on the temporary movement of natural persons: a simulation exercise, Journal of Economic Integration, 20 (4): 628-726.

White, R. (2007). An examination of the Danish immigrant-trade link, International Migration, 45 (5): 61-86. 
World Bank (2012). http://data.worldbank.org/indicator/PA.NUS.PPP URL (accessed 12 December 2012).

Wright, R., Ellis, M. and M. Reibel (1997). The linkage between immigration and internal migration in large metropolitan areas in the United States, Economic Geography, 73: 234-54.

Yilmazkuday, H. (2013). Mismeasurement of distance effects: the role of internal location of production, mimeo, Florida International University. 
VITA

\section{MICHAEL GOOD}

2009-2012

Presidential Fellowship

2010

M.A., Economics

Florida International University

2012

Research Grant

Horowitz Foundation for Social Policy

2012

Doctoral Evidence Acquisition Fellowship

2013-2014

Dissertation Year Fellowship

2014

Ph.D., Economics

Florida International University

\section{PUBLICATIONS AND PRESENTATIONS}

Good, M. (June 2012). XV Applied Economics Annual Meeting, La Coruña, Spain.

Good, M. (November 2012). Southern Economic Association Annual Meeting, New Orleans, Louisiana.

Good, M. (November 2012). Latin America and Caribbean Economic Association Meeting, Lima, Peru.

Good, M. (April 2013). $4^{\text {th }}$ NORFACE/CReAM Migration Conference, London, England.

Good, M. (2013). Do immigrant outflows lead to native inflows? An empirical analysis of the migratory responses to US state immigration legislation, Applied Economics, 45 (30): 4275-97.

Good, M. (2013). Gravity and localized migration, Economics Bulletin, 33 (4): 2445-53.

Good, M. (November 2013). Southern Economic Association Annual Meeting, Tampa, Florida, USA.

Good, M. (November 2013). Northeast Universities Development Consortium, Cambridge, Massachusetts, USA. 